\title{
CHARACTERIZING THE OCCURRENCE, MINERALOGY, GEOCHEMISTRY, AND \\ GEOCHRONOLOGY OF AN ASH BED IN THE VERULAM FORMATION (UPPER ORDOVICIAN), OTTAWA EMBAYMENT, EASTERN ONTARIO
}

\author{
Mohammad J. Al-Dulami \\ B.Sc. (University of Jordan, Jordan) \\ M.Sc. (University of Jordan, Jordan)
}

A thesis presented to

The Faculty of Graduate Studies and Research in partial fulfillment of the requirements for the degree of

\section{Master of Science}

\begin{abstract}
Department of Earth Sciences, Carleton University
Ottawa-Carleton Geoscience Centre
\end{abstract}

January, 2010

(C) Copyright 2010,

Mohammad J. Al-Dulami 


\section{ABSTRACT}

Two thin ( 4.5 and $2.5 \mathrm{~cm})$, very friable biotite-bearing shale beds separated by a $1-\mathrm{cm}$ thick crinoidal-brachiopod floatstone can be traced in two cores through the Upper Ordovician Verulam Formation (Ottawa Group) along a 24-km transect in the western portion of the Ottawa Embayment, eastern Ontario. A further $3 \mathrm{~km}$ northwest of this transect, in both core and outcrop, the beds are not present at the equivalent stratigraphic position, but trace biotite occurs in shale typical of the Verulam Formation. The friable shale beds contain a predominant (60-65\%) crystalline clay matrix of illite and minor kaolinite within which are the following components: abraded fragments $(<10 \%)$ of partially chlorite- and illite-altered biotite; elongate to rounded clay-lithic fragments (containing angular quartz and potassic feldspar and framobidal pyrite); aggregates of framboidal pyrite; rare skeletal carbonate; and, accessory mineralogy of phenocrystic apatite (defined by XRD and SEM), gypsum and feldspar (recognized by XRD only), and magnetite (black in hand specimen, defined by XRD). In the lower bed, biotite grains decrease abruptly to the NW along the $24-\mathrm{km}$ transect in both abundance $(<10 \%$ to trace amount) and grain size $(300-400 \mu \mathrm{m}$ to 50 to $75 \mu \mathrm{m})$. In the upper bed, there is a similar grain size and abundance to the southeast, and only trace amount of the silt-size biotite with increased admixture of carbonate particles and mud matrix to the northwest.

The presence of phenocrysts of biotite and rare apatite, and soft friable nature of these shales, is compatible with documented characteristics of several Upper Ordovician and Lower Silurian altered ash beds in continental shelf deposits of the periIapetan region. Geophysical correlation of the core transect reveals continuity of this 
bentonite interval over $1000 \mathrm{~km}$ along a SW-NE transsect from the Michigan Basin to southern Quebec. This continuity is likely a strike-parallel orientation given the observed NW-directed decrease in grain size. Thus, the magmatic source may lie to the ESE or SE, and geochemical proxies identify a trachyandesitic to rhyodacitic/dacite parent magmatic source within a collisional plate boundary setting, as was developing along eastern Laurentia in the Late Ordovician. Calculated Mg\# values from biotites within altered volcanic ash deposits in the Upper Ordovician post-Turinian through Lower Silurian successions of the peri-Iapetan region demonstrate a net decrease in magmatic fractionation, with the most pronounced change resolved, as defined by this study, to likely be coincident with foundering of the foreland carbonate platform. In addition, regional correlation demonstrates that the Verulam ash bed coincides with a marked step-back in the paleogeographic position of the Laurentian margin. These new observations further emphasize a strong linkage between the Taconic orogen and platform development in eastern Laurentia. 


\section{DEDICATION}

First, I would like to be grateful and thankful sincerely to our "GOD" for his care and blessing this work and helping me to finish it to be useful for the mankind, as a credit in balance of my good deeds, and the people who guide and help me, as well, therefore, I hope he accepts it from me.

Second, I dedicate this work to my Father and soul of died mom, brothers, sisters, my wife, and best friend Dr. Munim Al-Rawi, and to all the people who take care, support and encourage me.

Special dedication to my supervisor, Prof. George Dix., and all staff members, administrative staff and technicians in Department of Earth Sciences, Carleton University. I hope our “GOD” blesses them all, and blesses CANADA. 


\section{ACKNOWLEDGEMENTS}

I wish to express my deepest gratitude to my supervisor Prof. George Dix for this valuable advice, support and guidance to finish this work. I also want to express my gratitude to the staff members, technician and friends in the Department of Earth Sciences, Carleton University, Ottawa-Carleton Geoscience Centre.

I am grateful to the Drs. Mike Villeneuve and John Stirling, Geological survey of Canada, for their help in Ar-Ar dating and XRD analyses, respectively.

My sincere thanks are to Dr. Munim Al-Rawi, Chairman, Geology Department, Imperial College for his continuous support and encouragement during the stages of this work. 


\section{STATEMENT OF CONTRIBUTION}

The research was funded by an NSERC Discovery Grant to my supervisor, Dr. George R. Dix. A poster presentation of preliminary results was made at the 2008 GAC-MACAGU Meeting, Toronto. The style, language, and written presentation of this thesis reflect input from my supervisor. Collection of data, and its processing, define my own work, while its interpretation represents discussions with my supervisor. 


\section{TABLE OF CONTENTS}

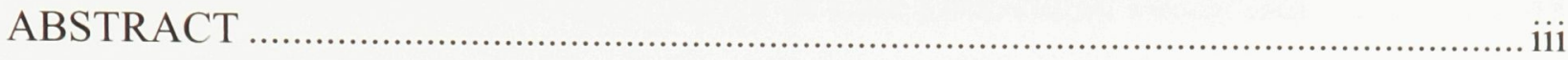

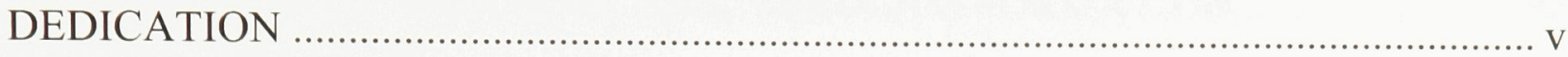

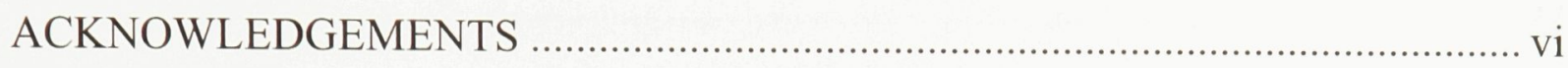

STATEMENT OF CONTRIBUTION ........................................................... vii

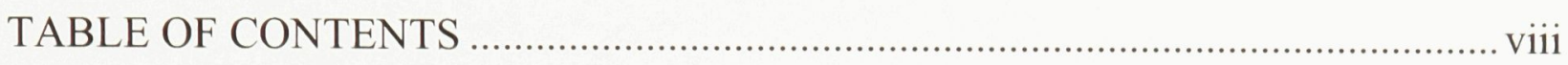

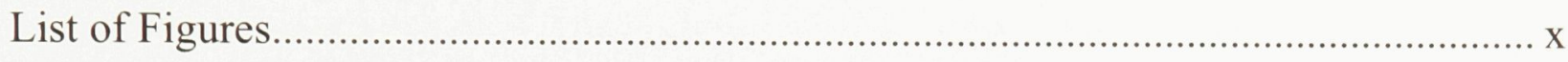

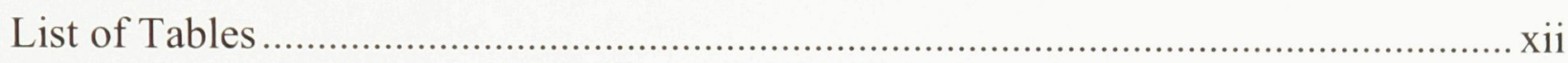

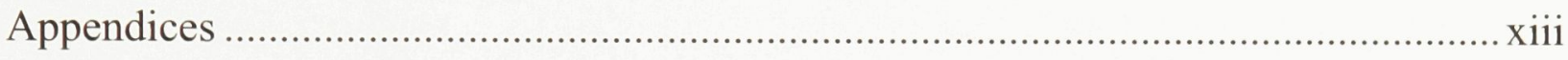

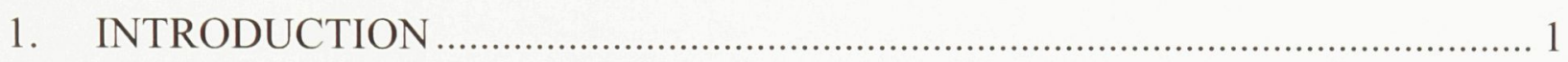

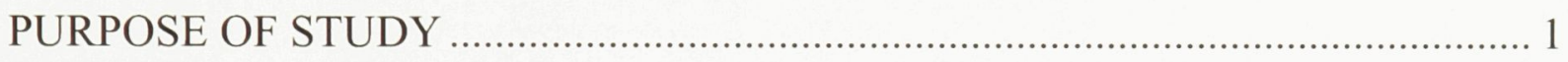

GEOLOGICAL SETTING ....................................................................... 5

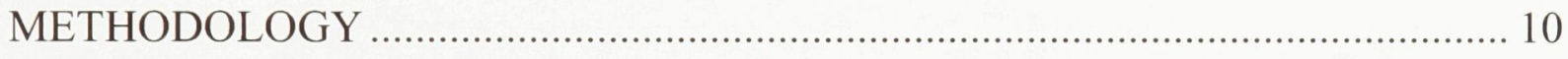

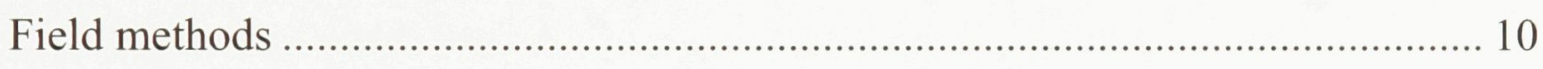

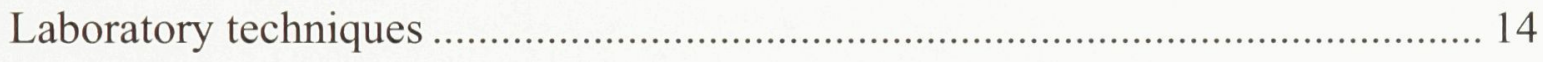

2. ANALYSIS OF THE VERULAM FRIABLE SHALE .................................. 18

DISTRIBUTION AND STRATIGRAPHIC FRAMEWORK .............................. 18

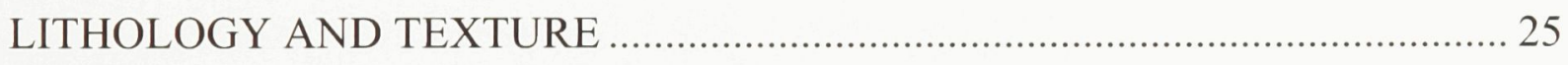

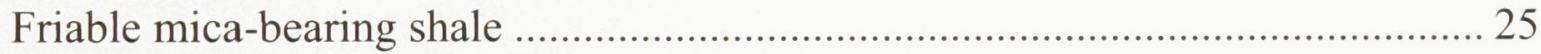

Billings Bridge and Lebreton core sites. No biotite was recovered from the Nepean site further supporting the northwest termination of biotite distribution. ............. 40

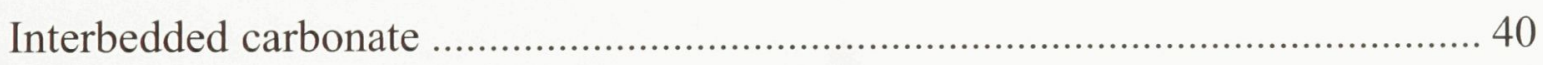

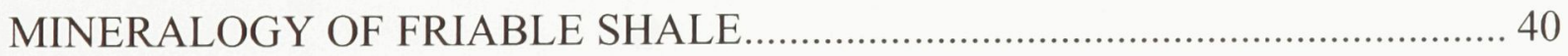

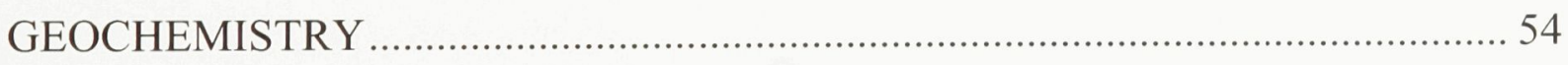

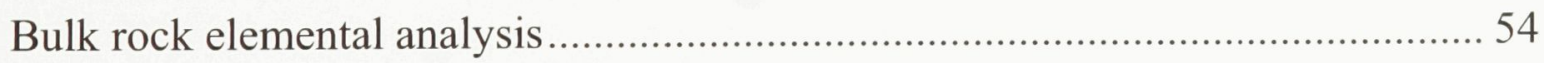

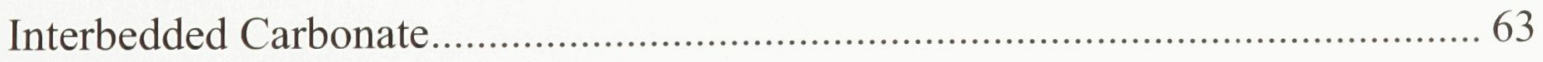

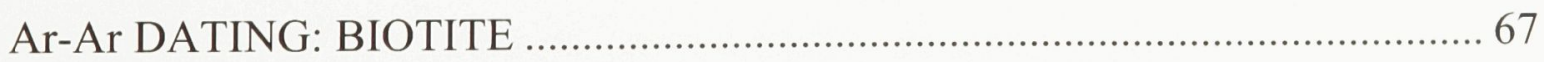

3. ORIGIN OF THE VERULAM FRIABLE SHALE ...................................... 72

THE VERULAM FRIABLE SHALES AS ALTERED VOLCANIC ASH............ 72

Stratigraphic and lithological evidence ................................................ 72 


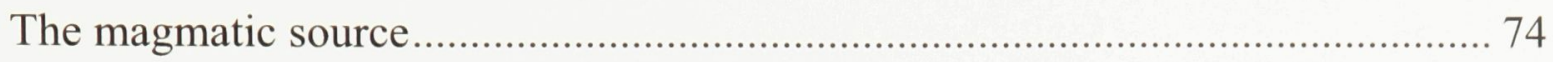

Comparison with peri-Iapetan Late Ordovician - Early Silurian volcanism ......... 76

New tectonostratigraphic significance of Late Ordovician volcanism....................8 88

4. DIAGENESIS OF VOLCANIC ASH, VERULAM FORMATION ......................... 92

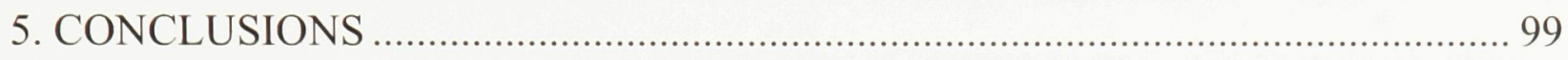

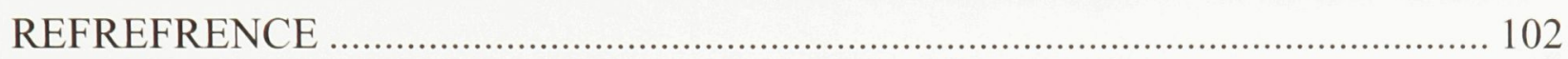




\section{List of Figures}

Figure 1. Global and temporal distribution of Ordovician K-bentonites. ..................... 2

Figure 2. General bedrock geology of the Ottawa Embayment, eastern Ontario............ 6

Figure 3. Detailed bedrock geology for the City of Ottawa region, and sample location sites

Figure 4. Lower Paleozoic stratigraphy for the Ottawa Embayment (eastern Ontario). 11

Figure 5. Lithostratigraphic succession and sample positions within the Russell, Billings

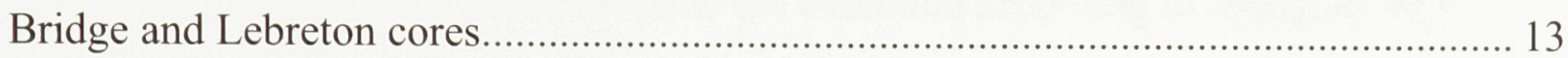

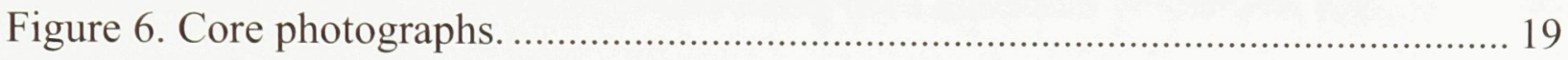

Figure 7. Local stratigraphy, Ottawa region, of the friable shale and equivalent compacted shale

Figure 8. Lithostratigraphic succession and sample positions, Nepean Point section. .. 23

Figure 9. Regional correlation of the friable shale interval.................................. 26

Figure 10. Grain size and type abundance........................................................ 29

Figure 11. Biotite abundance and grain size distribution.

Figure 12. Cumulative frequency of length/width (L/W) ratios for lithic fragments in Russell core.

Figure 13. Cumulative frequency of length/width (L/W) ratio for lithic fragments in the Billings Bridge core. 37

Figure 14. Photomicrograph of skeletal floatstone with grainstone to packstone matrix of carbonates rocks associated with the friable shale. 41

Figure 15. SEM photomicrographs of friable shale components. .......................... 43

Figure 16. X-ray diffractograms of oriented samples. ........................................ 45

Figure 17. Friable shale and compacted shale oxide geochemistry......................... 55

Figure 18. Pattern of REE-Chondrite for bulk ash samples of friable and compacted shales showing the enrichment in LREE over HREE.

Figure 19. $\delta{ }^{18} \mathrm{O}$ and $\delta{ }^{13} \mathrm{C}$ values of bulk sample consisting of mixed brachiopods and interstitial cement.

Figure 20: Bulk-rock elemental and stable isotope data, Verulam limestone.............. 68

Figure 21. Failure age-dating using Ar-Ar method of biotite in friable shales. 70 
Figure 22. Tectonomagmatic discrimination diagrams of A) biotite and B-D) bulk rock samples

Figure 23 Postulated areal distribution of the Millbrig + Kinnekulle events in the periIapetan region.

Figure 24. Bivariate plots of oxides showing distribution of the Millbrig, Deicke, and Kinnekulle events compared with the Verulam dataset. 82

Figure 25. Biotite geochemistry relative to age of volcanic event, Late Ordovician to Early Silurian, peri-Iapetan region. 84

Figure 26. Magmatic stages of volcanic arc evolution according to changing Mg\# associated with micas in volcanic events along the Laurentian continental margin..... 90

Figure 27 Clay mineral products relative to rock types and magma source. 93

Figure 28 Chlorite and illite formation by alteration of biotite. 97 


\section{List of Tables}

Table 1. Carbonate and mineralogical grain size distribution of cores. ...................... 31

Table 2. Length (L), width (W) and (L/W) ratios of lithic fragments in friable shale... 39

Table 3. Elemental composition of biotite, illite and chlorite in friable shale samples. 47

Table 4. Mineraloglogy of bulk and clay mineral in friable \& compacted shales. ........ 50

Table 5. Relative distribution of clay minerals. . ..................................................... 53

Table 6. Selected Major oxides and ratios of bulk friable and compacted shales........ 60

Table 7. $\delta^{18} \mathrm{O}$ and $\delta{ }^{13} \mathrm{C}$ values of the carbonate in Russel \& Billings Bridge core..... 66

Table 8. Biotite geochemistry of K-bentonite in Canada, North America \& Europe. ... 86

Table 9. Dataset of Upper Ordovician \& Lower Silurian of peri-Iapetan K-bentonite. .87 


\section{Appendices}

APPENDIX A : Length (L), width (W), and L/W ratios of lithic fragments in lower ash bed, Russell core 107

APPENDIX B: Trace Elements data ............................................................. 117

APPENDIX C: Rare Earth Elements data.................................................. 119

APPENDIX D: HFSE, LILE and REE dataset of friable and compacted shales,

Verulam formation.

APPENDIX E : Selected trace element correlation of bulk samples of friable (blue) and compacted shales (red). 121

APPENDIX F. Normalised Major Oxide without LOI. 122 


\section{INTRODUCTION}

\section{PURPOSE OF STUDY}

Many Lower Paleozoic shallow-shelf to deep-basin facies contain altered pyroclastic beds known as K-bentonites (Fig. 1). They are relatively thin (usually $\mathrm{cm}$ to dem in thickness), yet can be laterally continuous to discontinuous over hundreds of kilometres. In eastern North America, Upper Ordovician (Mohawkian) K-bentonites are prominent within the shallow-water carbonate platform succession of the Taconic foreland basins (Kay, 1931, 1935; 1942; Huff et al. 1992; 1996; Huff, 2008; Kolata et al., 1996). These beds represent single or multiple fallout deposits ranging between andesitic to rhyolitic endmember compositions. The source for these deposits is interpreted to have been the developing volcanic arc positioned hundreds of kilometres to the east of the paleoshelf environment (Huff et al., 1996).

K-bentonites are recorded from the younger shale-basin successions of Cincinnatian/Ashgillian age that formed following foundering of the regional carbonate shelf systems (Berkley and Baird, 2002; Sharma et al., 2005). Their composition and geochemistry are less well known. In the Geological Survey of Canada (GSC) Russell core, in eastern Ontario, a K-bentonite coincides with the base of the Taconic flysch succession within the Billings Formation, the lowest unit of the Taconic (Late Ordovician) shale basin. On the basis of mica (phlogopite) composition and other proxies, the source of the altered ash was interpreted to have been a magma more basic than those typical of Upper Ordovician K-bentonites that occur within the underlying foreland carbonate platform succession, yet similar to Early Silurian K-bentonites of Scandinavia. The Sharma et al. (2005) suggested that this difference may reflect a new 
Figure 1. Global and temporal distribution of Ordovician K-bentonites.

Modified from Huff (2008). 


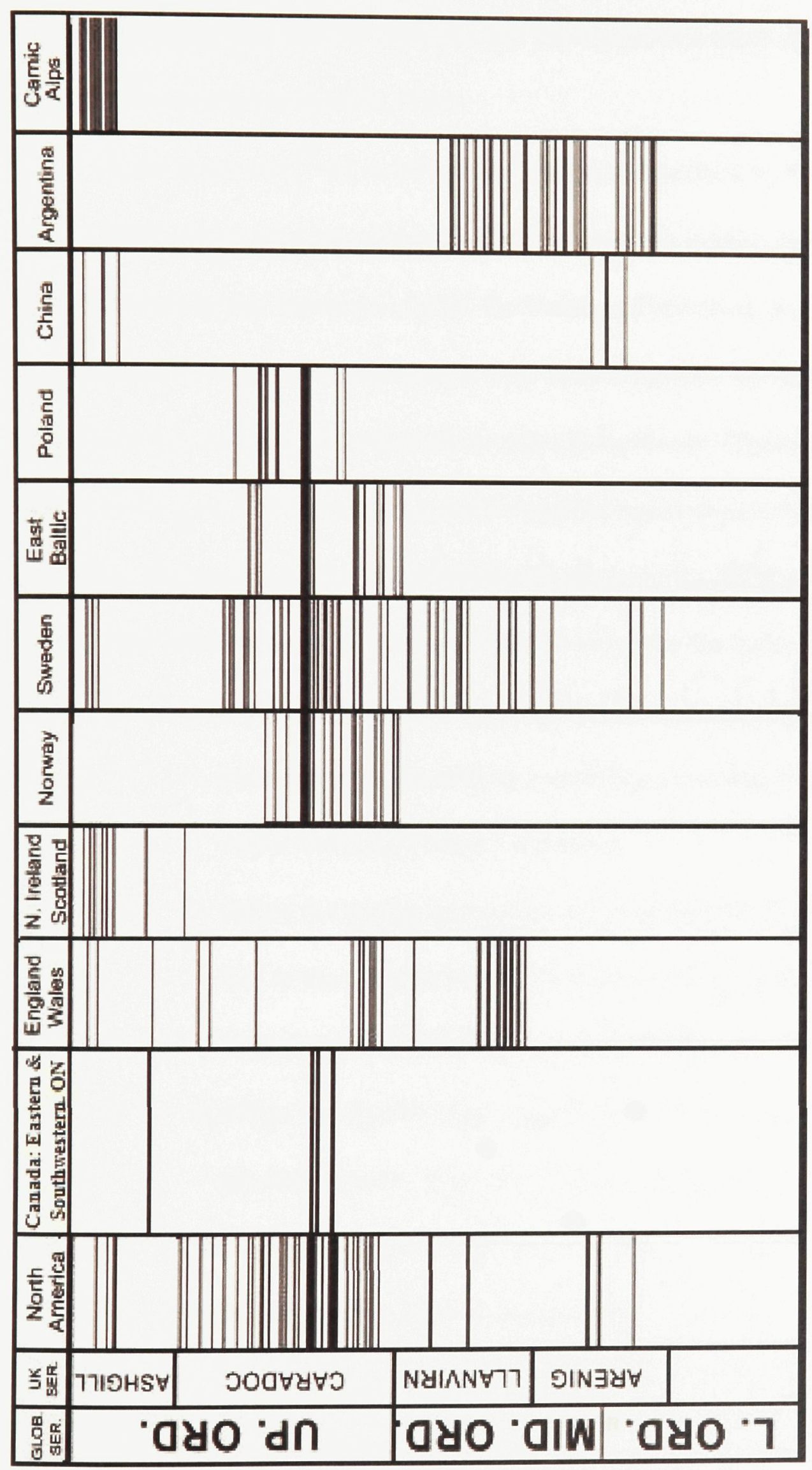


stage of orogenic magmatism along eastern North America, possibly driving the change in basin oceanography and fill, and one that incorporated a more significant volume of continentally-derived magmatic fluids.

In the same core, $\sim 35 \mathrm{~m}$ below the bentonite described by Sharma et al. (2005), Truax (2008) discovered two thin mica-bearing, soft and highly, friable shales separated by a thin fossiliferous limestone within the Verulam Formation, part of the regional foreland carbonate platform succession. The mica fragments are dark brown, and were interpreted (on the basis of field analysis only) to be biotite. Physically, these shales are similar to the Late Ordovician altered-ash deposits reported as K-bentonites within equivalent limestone successions of eastern North America (Huff et al., 1992).

This discovery allows the present study to pursue the following:

1) to evaluate the local to regional lateral extent of the altered ash beds within eastern Ontario by examining cores and outcrops that span the upper Verulam Formation interval;

2) to define the texture and composition of the altered ash beds, and relate this to the possible magmatic source and processes influencing their deposition and preservation within a carbonate shelf sea;

3) to define the absolute age of the biotite, thereby offering a precise geochronometric framework for the Verulam Formation; and,

4) to establish whether there was a significant change in bentonite (and-or biotite) composition across the platform-to-shale-basin boundary as recorded by the close proximity $(\sim 35 \mathrm{~m})$ of the ash beds in the upper Verulam and Billings formations. 


\section{GEOLOGICAL SETTING}

The Ottawa Embayment is a closed structural depression (Sanford, 1993) that cuts across the regional NE-SW Precambrian fabric of the Grenville Province, and is bounded by the Frontenac and Beauharnois arches, and the Adirondack Highlands (Fig. 2). The present shape of the Ottawa Embayment reflects structural control arising from formation of the Ottawa-Bonnechère Graben, a fault system interpreted to reflect partial reactivation of a crustal-scale Neoprotozoic fault system (Kumarapeli and Saull, 1966; Kumarapeli, 1985; Mereu et al. 1986). The study area (Fig. 3) lies within and borders (to the SE) the City of Ottawa, which lies within the axis of the graben. The earliest documented age of local faulting and seismicity in the Phanerozoic is of Middle Ordovician age coinciding with initiation of the distal Taconic foreland basin (Dix and Al-Rodhan, 2006), with seismic activity also occurring episodically during the Late Ordovician (Truax, 2008; Dix, unpublished data). Present-day faults and differential preservation of Lower Paleozoic strata within the embayment (Ontario Geological Survey, 1991) reflect a protracted, if episodic, tectonic history spanning the Phanerozoic (Sanford, 1993; Dix and Robinson, 2003; Romando and Benn, 2005).

During the Late Ordovician, this region formed an interior extension of the epicontinental St. Lawrence Platform that lay along eastern Laurentia (Salad Hersi and Dix, 1999). The regional Upper Ordovician lithostratigraphic framework of Ottawa Embayment is currently under review (Dix, unpublished data). However, the Lower Palaeozoic succession contains the preserved remnants of two cratonic sequences (Sloss, 1963): (1) the upper Sauk sequence, represented by a siliciclastic to mostly dolostone succession (Fig. 2), corresponds to restricted platform interior facies along the trailing 
Figure 2. General bedrock geology of the Ottawa Embayment, eastern Ontario.Based on Williams (1992). The blue area includes the sites of the GSC Russell, GSC Billings Bridge, and GSC Lebreton cores, and the outcrop section at Nepean Point. 


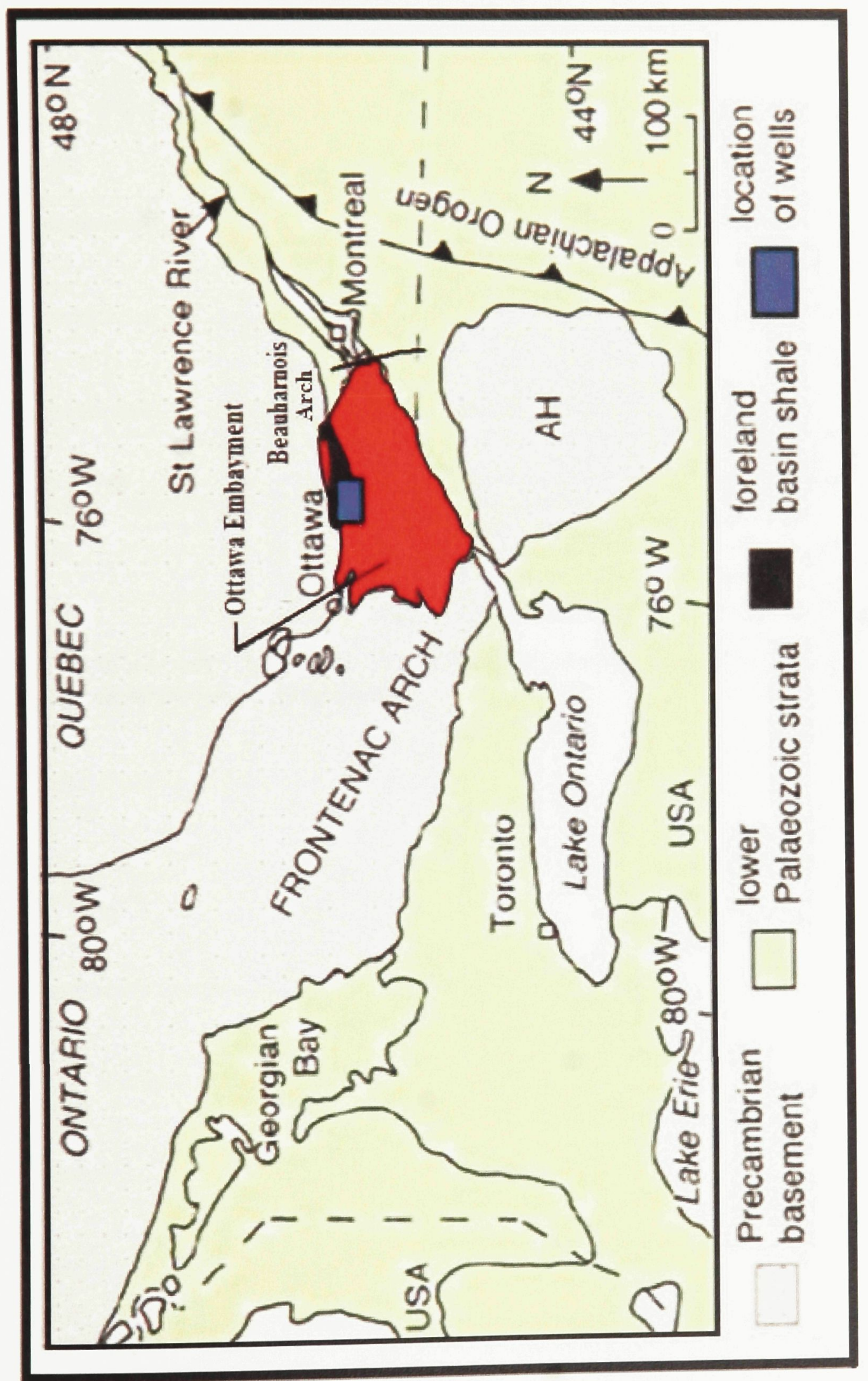


Figure 3. Detailed bedrock geology for the City of Ottawa region, and sample location sites. Based on www.ottawamap.com. 


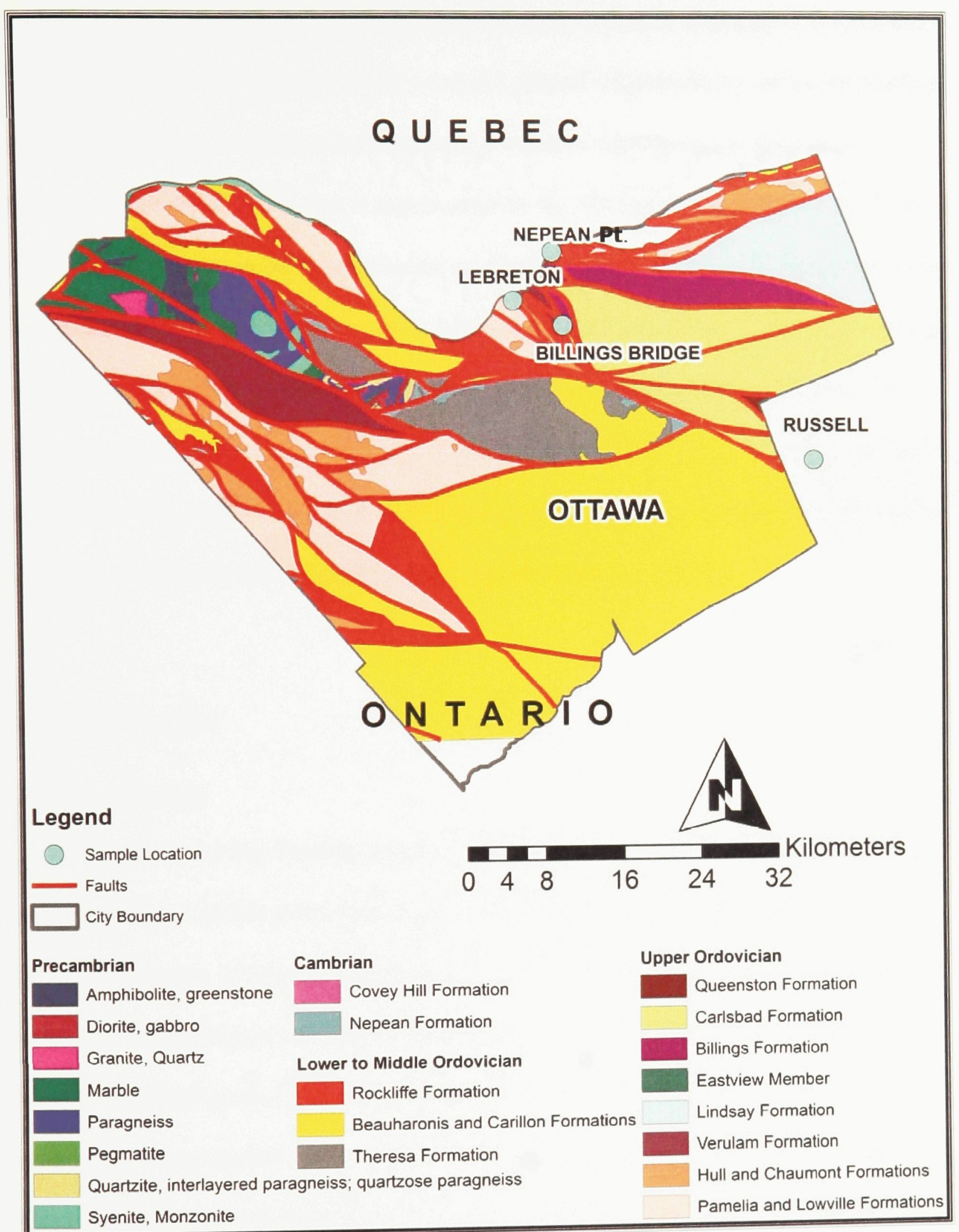


epicontinental St. Lawrence Platform facing the Iapetan Ocean basin; and (2) the lower Tippecanoe sequence, represented by a second general siliciclastic-to-carbonate platform succession (Fig. 4), corresponds to deposition within a developing foreland basin. Prominent carbonate deposition is represented by the Ottawa Group limestones, of which the Verulam Formation (that contains the studied stratigraphic interval), represents stormdominated shelf facies (Kiernan, 1999). Ultimately, carbonate shelf foundering at the end of the time of Lindsay Formation deposition led to the formation of an initial anoxic deep-water shale basin (lower Billings Bridge Formation; Sharma et al., 2003), but one which then filled rapidly deposition of flysch as represented by the upper Billings Bridge, Carlsbad, and Queenston formations (Fig. 4; Sharma et al., 2003).

\section{METHODOLOGY}

\section{Field methods}

A search for the Verulam ash-bed couplet defined by Truax (2008) was carried out examining available cores from eastern Ontario (Williams, 1991) housed at the Geological Survey of Canada's (GSC) archive and storage facility, Tunney's Pasture. The cored interval of interest was logged noting lithological and fossil content. Samples were taken for mineralogical, geochemical and petrographic analyses, their distribution panning the target interval (Fig. 5). Samples of the typical Ordovician shale that lay both above and below the interpreted ash-bed interval were also sampled. The Billings Bridge and Russell cores are sawn in half, with only one half archived. As only one half of this material could be sampled, and given that the ash-bed couplet is only $\sim 6 \mathrm{~cm}$ thick, and 
Figure 4. Lower Paleozoic stratigraphy for the Ottawa Embayment (eastern Ontario). Modified from Dix and Al-Rodhan (2006). 


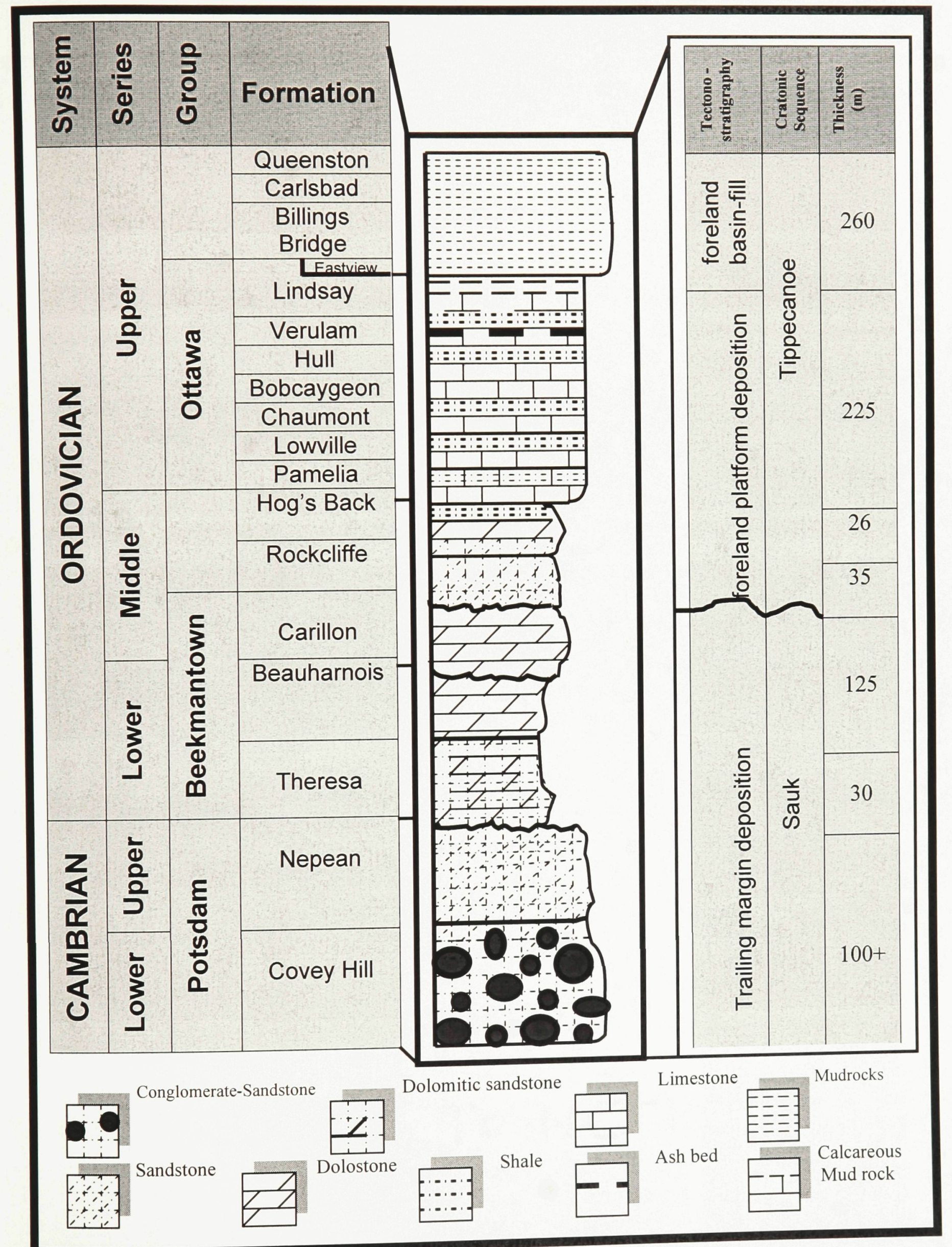


Figure 5. Lithostratigraphic succession and sample positions within the Russell, Billings Bridge and Lebreton cores. 

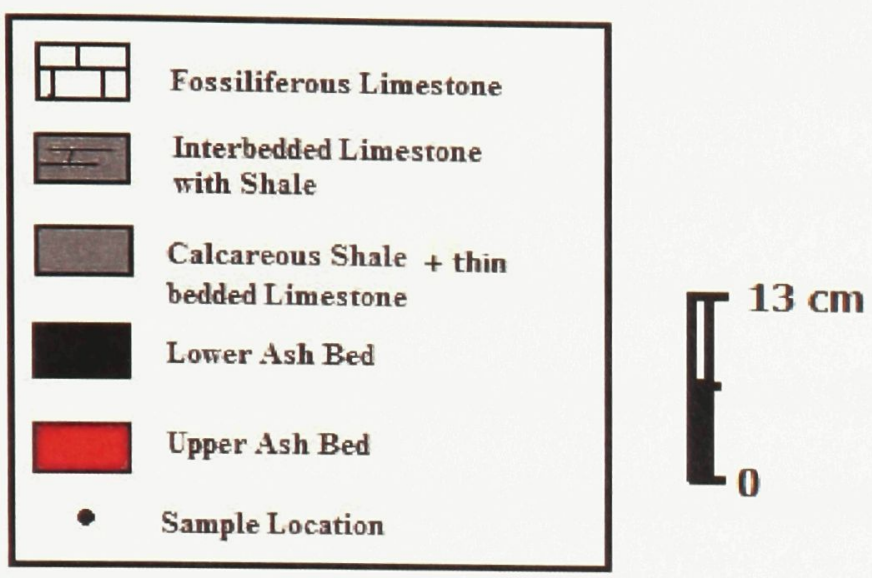

Leb.15

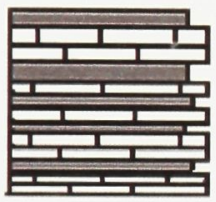

$-3 \mathrm{Km}$

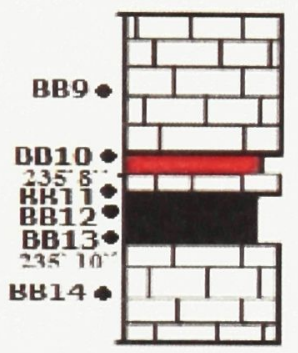

Lebreton Core

NW

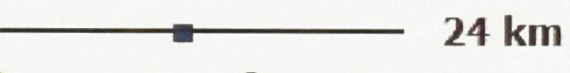

Billings Bridge Core

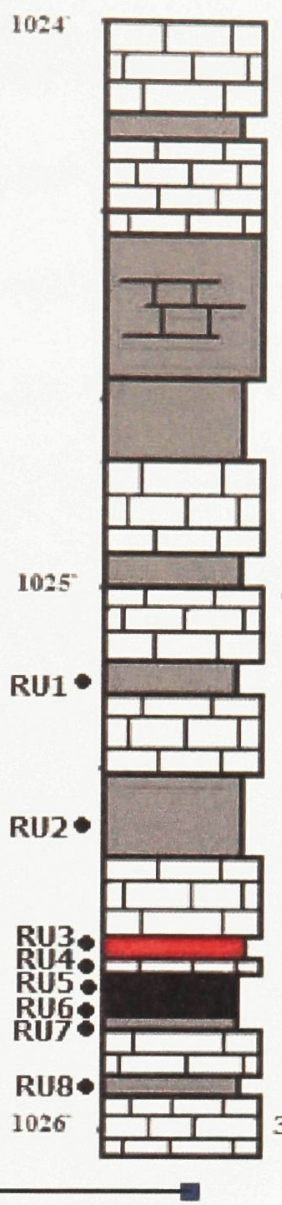

312.725

Russell Core

\begin{tabular}{|c|c|c|c|}
\hline Core & Sample No. & Depth (ft.) & Depth $(\mathrm{cm})$ \\
\hline \multicolumn{4}{|l|}{ Russell: } \\
\hline \multirow[t]{7}{*}{ (Upper) } & RU1 & $1025^{\prime} 2^{\prime \prime}$ & 312.471 \\
\hline & RU2 & $1025^{\prime \prime} 5^{\prime \prime}$ & 312.547 \\
\hline & RU3 & $1025^{\prime} 6.25^{\prime \prime}-7^{\prime \prime}$ & $312.579-312.598$ \\
\hline & RU4 & $1025^{\prime} 7^{\prime \prime}$ & 312.598 \\
\hline & RU5 & $1025^{\prime} 8.5^{\prime \prime}-7.5^{\prime \prime}$ & $312.636-312.611$ \\
\hline & RU6 & $1025^{\prime} 8.5^{\prime \prime}-9.5^{\prime \prime}$ & $312.636-312.661$ \\
\hline & RU7 & $1025^{\prime} 9.5^{\prime \prime}$ & 312.661 \\
\hline (Lower) & RU8 & $1025^{\prime \prime} 10.5^{\prime \prime}$ & 312.687 \\
\hline \multicolumn{4}{|c|}{ Brllings Bridge: } \\
\hline \multirow[t]{5}{*}{ (Upper) } & $\mathrm{BB9}$ & $235^{\prime} 6.5^{\prime \prime}-7^{\prime \prime}$ & $71.973-71.806$ \\
\hline & B日10 & $235^{\prime \prime} 7^{\prime \prime}-8^{\prime \prime}$ & $71.806-71.831$ \\
\hline & $\mathrm{BB} 11$ & $235^{\prime} 8^{\prime \prime}-83 / 4^{\prime \prime}$ & $71.831-71.850$ \\
\hline & $\mathrm{BB} 12$ & $235^{\prime} 83 / 4^{\prime \prime}-10^{\prime \prime}$ & $71.850-71.882$ \\
\hline & $\mathrm{BB} 13$ & $235^{\prime} 83 / 4^{\prime \prime}$ & 71.850 \\
\hline (Lower) & BB14 & $235^{\prime} 10^{\prime \prime}$ & 71.895 \\
\hline \multicolumn{4}{|l|}{ Lebreton: } \\
\hline & Leb. 15 & $20^{\prime}$ & 50.8 \\
\hline
\end{tabular}


bulk samples, following addition of $10 \% \mathrm{HCl}$ acid in order to dissolve primary or diagenetic carbonate, neither of which reflect the original ash source. Visual examination showed that the predominant grain size was less than about $80 \mu \mathrm{m}$. Thus, wet sieving used a $63 \mu \mathrm{m}$ mesh to separate fine-sand from the mud fraction, and a $75 \mu \mathrm{m}$ mesh to detail size frequency within the sand fraction. Coarser-grained materials (e.g., biotite) were eventually handpicked. Grain size frequency was determined, along with mineralogical content. From the fine-sand separates, there appear to be lithic grains that may have been altered pumice, and provided the opportunity to evaluate length / width ratios from microphotographs for comparison with modern volcanic pyroclastic systems.

Slurries of powdered whole-rock samples and the $<2$-micron fraction, separated by centrifuge, were pipetted onto a glass slide for x-ray diffractometry. In total, twentythree slides were prepared and analysed at the Geological Survey of Canada (Ottawa) using a Bruker D8 Advance Powder, with settings of 40KV, 40mA, Co-Ka radiation $(\lambda=1.793)$, over a 2-60 2-theta degree scan range, at a scanning speed of 2 degrees 2-theta per minute with a chart speed of $2 \mathrm{~cm} / \mathrm{min}$. For each of the above samples, three slides were prepared; the first, as described above, and referred to as "normal"; the second prepared as above but then glycolated (treated with ethylene glycol) for two hours; and, the third, was prepared then heated starting at $400 \mathrm{C}^{\circ}$, for at least half hour, to $550^{\circ} \mathrm{C}$ and kept at this temperature for half and hour as well.

Given the small amount of material available from the cores, major element analysis was carried out on $0.5 \mathrm{~g}$ of whole-rock powder by Activation Laboratories Ltd., Ancaster, ON. The procedure involved use of a Phillips PW-2400 sequential XRF analyzer with Phillips Super Q software (Norrish, 1969). Cited detection limits are 0.01 
$\%$, and the reader is directed to their website (www.actlabs.com). Trace element and rare earth concentrations were determined from whole-rock powdered samples at the Ontario Geological Survey (Sudbury) laboratories. Their method codes are designated IM100/101. Detection limits vary by element, and are cited in the Appendix.

Quantitative elemental spot analyses of biotite, chlorite and illite were carried out on an automated 4-spectrometer Camera Camebax MBX electron probe by wavelength dispersive $\mathrm{X}$-ray analysis (WDX). Operating conditions were set at $15 \mathrm{KV}$, and 20 nanoamperes beam current. Scanning Electron Microscope (SEM) for qualitative assessment of elemental geochemistry of various minerals.

Cathodoluminescence attributes of the carbonate bed that lay between and bracket the ash beds within the Russell and Billings Bridge cores was examined using an ELM-2 luminoscope (set at 0.5 milliamps, $12 \mathrm{kV}$, and $<40$ millitorr) to help differentiate carbonate paragenesis. From this, calcite cement and brachiopod shell material were independently sampled (using a dental microdrill) for $\delta^{13} \mathrm{C}$ and $\delta^{18} \mathrm{O}$ isotope analysis at the University of Ottawa. The sample is mixed with $0.1 \mathrm{ml}$ of $\mathrm{H}_{3} \mathrm{PO}_{4}$, capped and heliumflushed while the container horizontal. Measurements were taken at $25 \mathrm{C}^{\circ}$ within $24 \mathrm{hrs}$ was performed on Delta XP and Gas Bench II from Thermo Finnigan, with precision (2 $\sigma$ ) \pm 0.1 permil, and then, normalized using international standards NBS-18, NBS-19, and LSVEC.

Biotite crystals within the ash beds offered the potential for absolute age-date analysis by the Ar-Ar method. A window of opportunity arose to submit samples for quick turnaround, but this meant that material was submitted prior to SEM examination of the biotite's texture or its composition apart from examination in a binocular 
microscope. Analysis was carried out under the direction of the Geological Survey of Canada. Biotite grains were hand picked from the Russell core, the best site, then divided according to their grain size frequency. The largest and visually least-altered biotite grains were cleaned with acetone, and then submitted for $\mathrm{Ar}^{39} / \mathrm{Ar}^{40}$ analysis that requires use of the McMaster University nuclear reactor. The process failed due to extensive alteration of the biotite, not otherwise visually evident at the time, but obvious with later electron microscopic analysis. 


\section{ANALYSIS OF THE VERULAM FRIABLE SHALE}

\section{DISTRIBUTION AND STRATIGRAPHIC FRAMEWORK}

In southern and eastern Ontario, the Verulam Formation is characterized by interbedded fossiliferous limestone and dark grey, well-compacted shale (Liberty, 1969; Williams, 1991). In contrast to this background lithology, however, two thin, highly friable, light grey shales with dark flakes of a mica visually similar to biotite occur within the upper Verulam Formation as recorded from the GSC Billings Bridge and GSC Russell cores (Truax, 2008), the two sites located about $24 \mathrm{~km}$ apart within and southeast of the City of Ottawa (Fig. 3). The lower shale is only $\sim 4.5 \mathrm{~cm}$ thick, and the upper shale is $\sim 2.5 \mathrm{~cm}$ thick; in each core, these are separated by a thin $(\sim 1 \mathrm{~cm})$ bed of fossiliferous limestone (Fig. 5 and $6 \mathrm{~A} \& \mathrm{~B}$ ). There is a notable increase in abundance of skeletal carbonate (including crinoid ossicles) mixed into the upper shale bed in the Billings Bridge core.

About $3 \mathrm{~km}$ northwest of the Billings Bridge site is the location of the GSC Lebreton core (Fig. 3). On the basis of a regional stratigraphic distribution of shale-bed density within the Verulam Formation (Fig. 7), the predicted stratigraphic position within the Lebreton core was determined, but the anomalous friable shale does not occur. Instead, there are only well-compacted dark grey shales (typical of the Verulam Formation) thinly interbedded with limestone (Fig. 6C). Using the same stratigraphic approach, the stratigraphic level at which the anomalous shales should occur in the Nepean Point section (Fig. 3) was identified (Fig. 7 and 8). However, only shales typical of the Verulam Formation are interbedded with limestone. 
Figure 6. Core photographs. The absence of altered ash beds in the Lebreton core (A) is in contrast to friable shale in the Billings Bridge (B) and Russell (C) cores at the interpreted same stratigraphic position. 

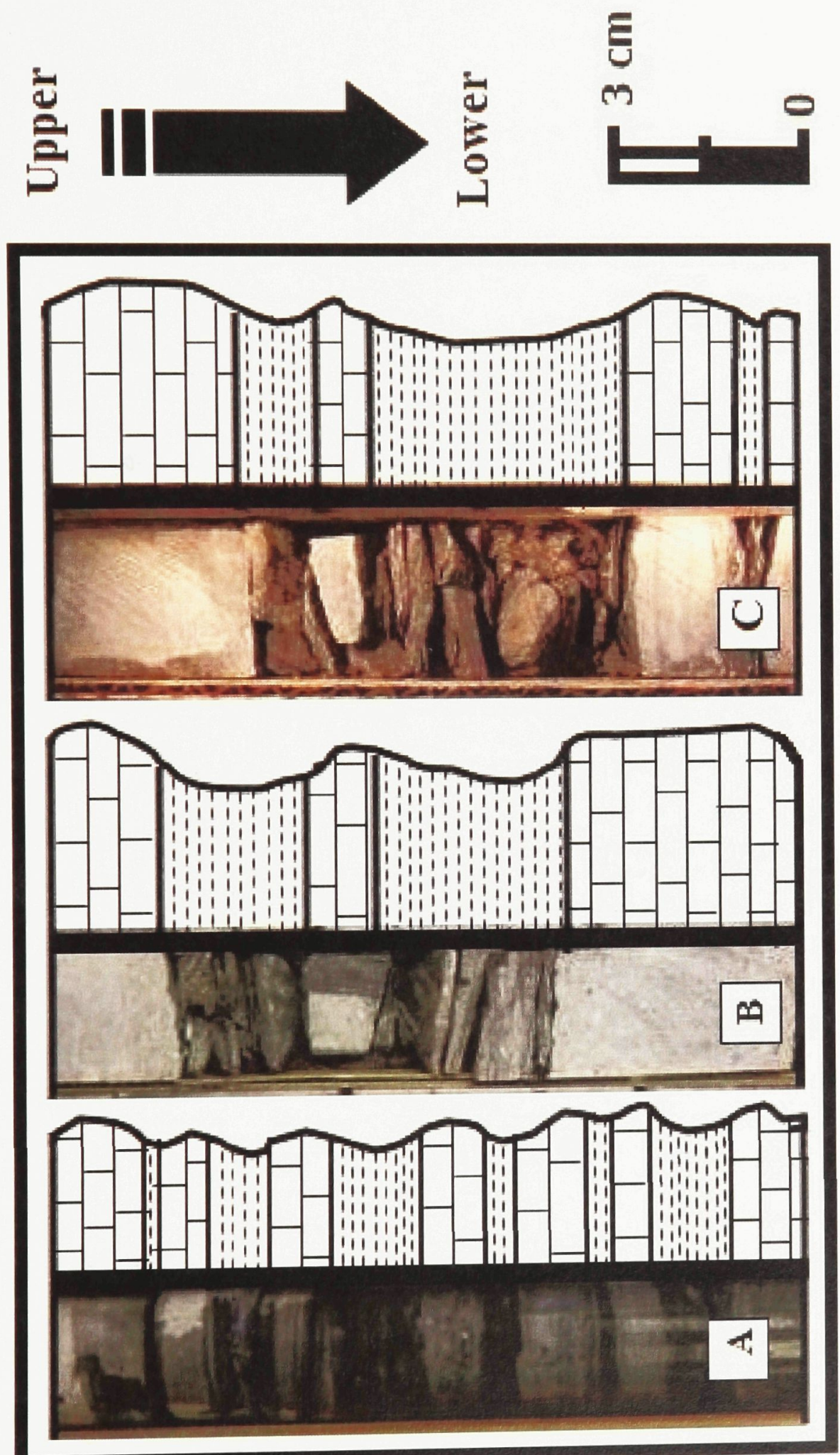
Figure 7. Local stratigraphy, Ottawa region, of the friable shale and equivalent compacted shale. This is compared to the shale density within the formation with shales thicker than $1 \mathrm{~cm}$ indicated by black bars. From Truax (2008) and this study. 


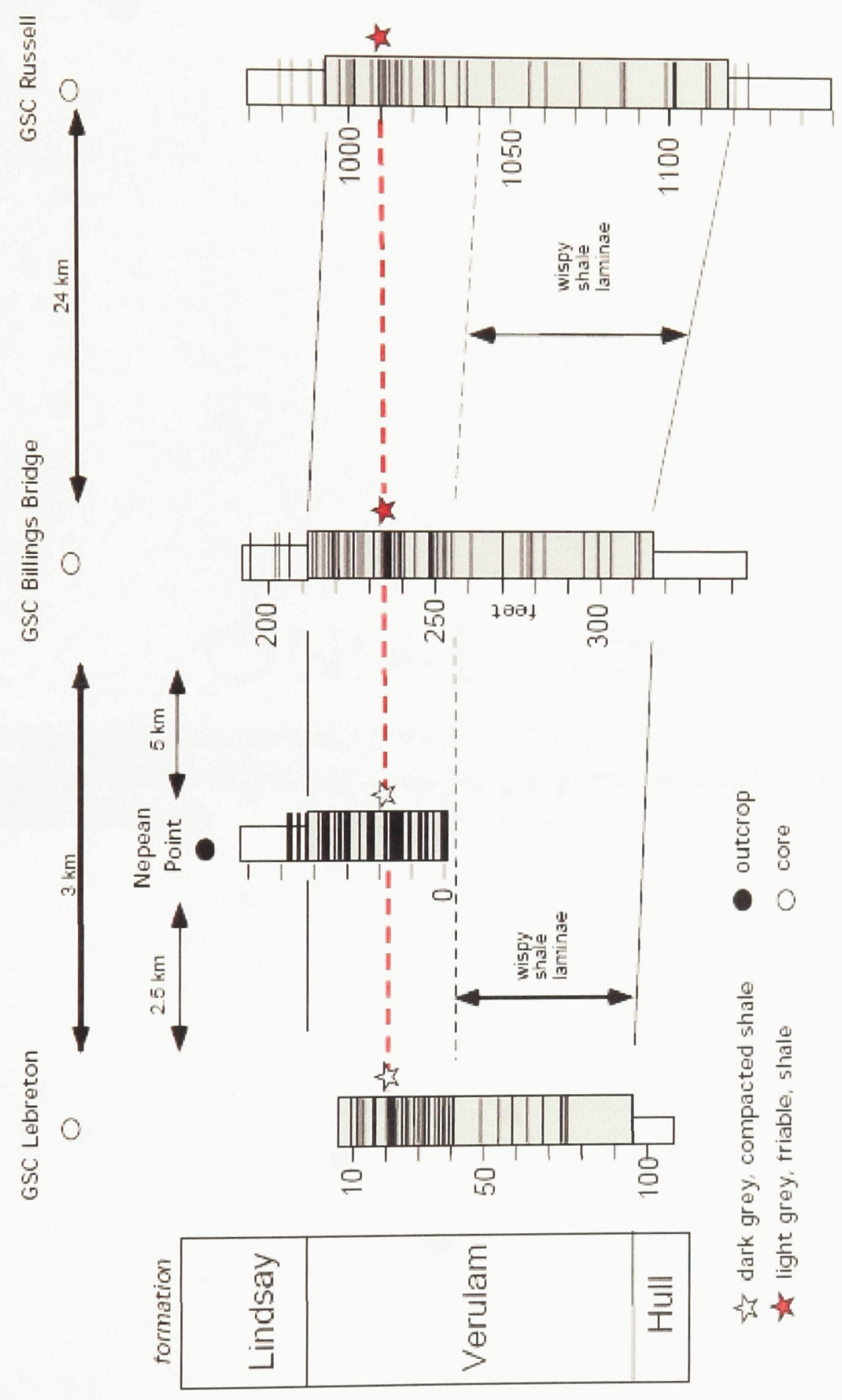


Figure 8. Lithostratigraphic succession and sample positions, Nepean Point section. Only shale and nodular limestone are indicated by patterns, the remaining section is composed of skeletal limestone. 


\section{Nepean Point Section}
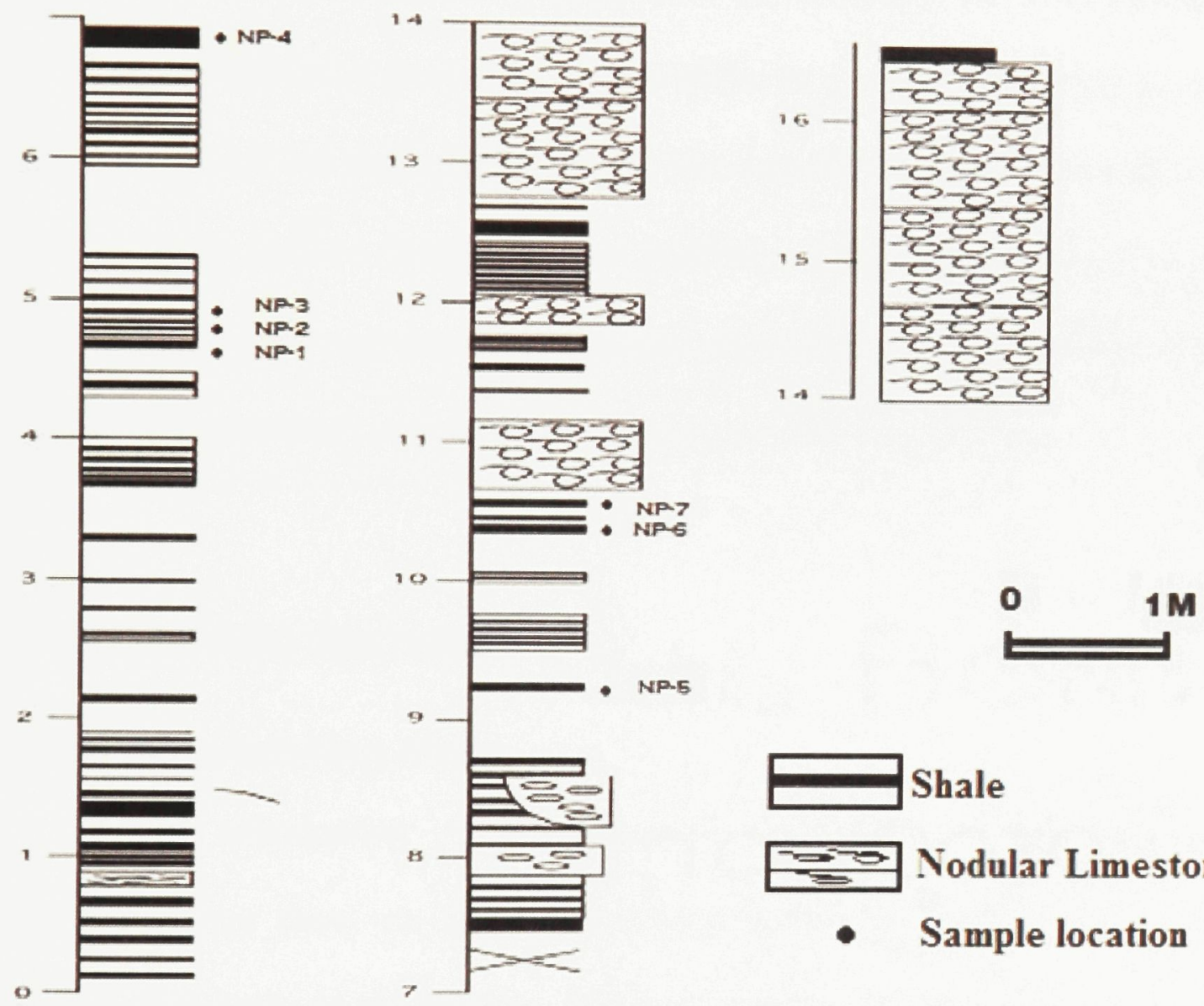

$=$ Nodular Limestone

- Sample location 
The twin friable shales in the Russell core occur about $10 \mathrm{~m}$ below the top of the Verulam Formation. This is the approximate stratigraphic level at which an altered ash bed (Kbentonite) was described in outcrop from central Ontario by Liberty (1969), positioned about $27 \mathrm{ft}(\sim 8 \mathrm{~m})$ below the top of this formation. On the basis of geophysical well $\log$ (gamma ray) SW-NE transect extending from the Michigan Basin to southern Quebec (Fig. 9), the stratigraphic level of the friable shales in the Russell core appear to correlate with a documented ash bed in the Michigan Basin (Kolata et al., 1996), and a possible ash bed in southern Quebec (Bergström and Mitchell, 1994). Of note, however, this same stratigraphic level corresponds to a prominent westward shift of the Laurentian carbonate platform-basin transition now preserved in southern Quebec (Fig. 9).

\section{LITHOLOGY AND TEXTURE}

\section{Friable mica-bearing shale}

Visual examination of the core with hand lens and binocular microscopy demonstrates that there are abrupt boundaries between the basal shale bed and the bounding limestones in the Billings Bridge and Russell cores. However, the boundary between the upper shale bed and the overlying limestones is gradational: in the Billings Bridge core, sample BB13 (Fig. 5) reveals a relatively common presence of skeletal carbonate (including crinoid ossicles, brachiopod shells); in the Russell core, the upper friable shale bed (samples RU 5 and 6) is overlain by "normal" shale (sample RU 7), then limestone. The friable shales are light grey, speckled with dark brown mica flakes. 
Figure 9. Regional correlation of the friable shale interval. Based on geophysical well logs (gamma ray), the friable shale of the Verulam Formation is correlated with a wireline log and lithology changes that can be traced over $\sim 1000 \mathrm{~km}$ from the Michigan Basin to southern Quebec according to comparison with previously published transects. 
$\hat{N}$

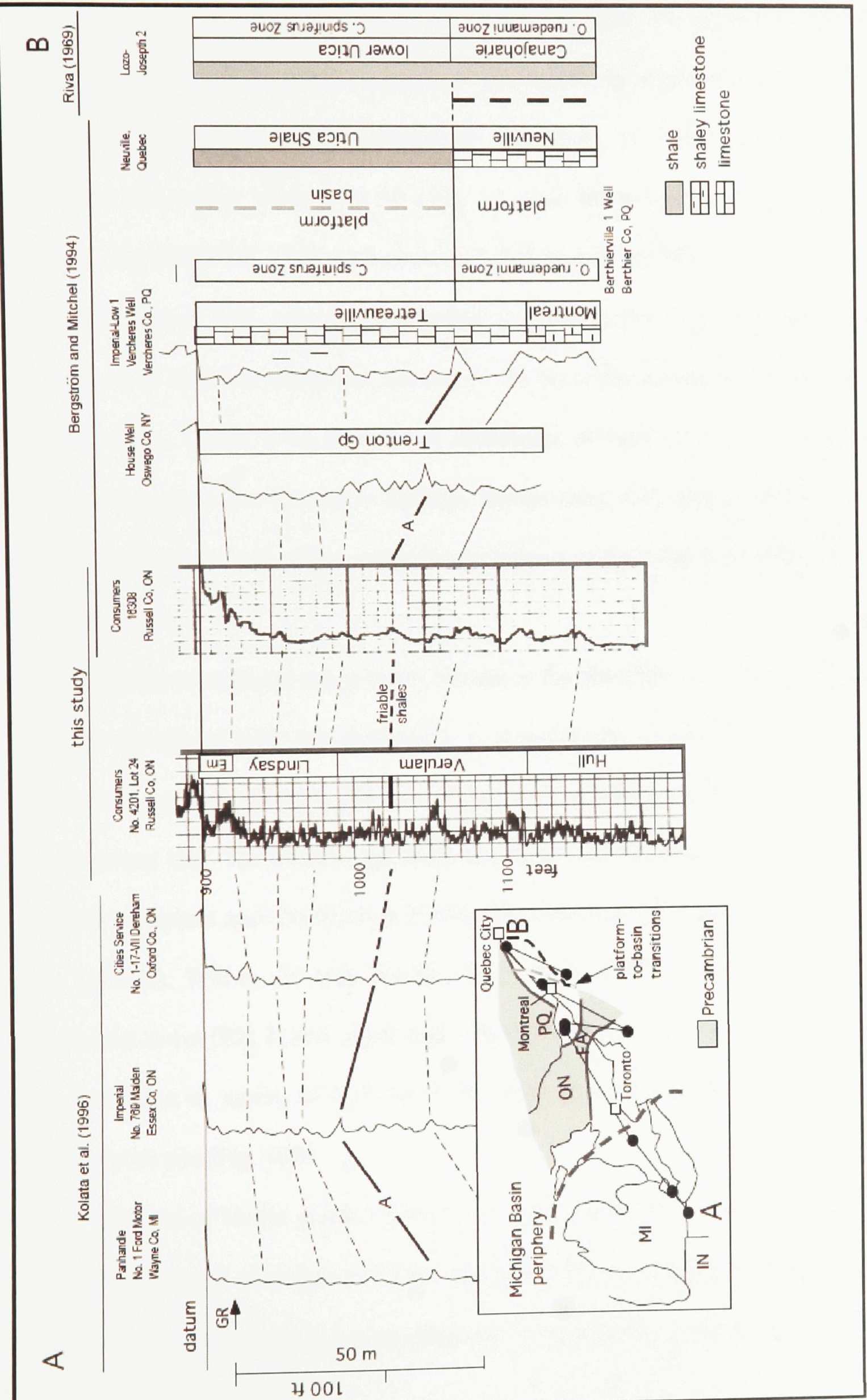


They are soft and highly friable, and easily disaggregate in water. With binocular microscopy, the ash bulk sample consists of the following components: silt to sand-size anhedral to subhedral (pseudohexagonal) fragments of dark brown, red-brown, colourless, and yellow mica $(<10 \%$; (Fig. 11 A \& B); anhedral silt to fine-sand-size grains of clay (60-65\%), often with contained dark brown crystals or fragments; rounded clumps of pyrite $(<5 \%)$; anhedral to euhedral opaque material (trace amounts); magnetite and gypsum in trace amounts; and, the rest $(\sim 20 \%)$ of the material consists of euhedral crystals of clay. There is an increase in abundance of light yellow to colourless mica flakes moving from the Russell to Billings Bridge sites, and, stratigraphically from the lower to upper shale beds. SEM analysis (see below) reveals that these have been altered to clay minerals.

In order to evaluate any primary change in the abundance of primary silicates that might be associated with ash deposition, it is necessary to remove the carbonate (by dissolution) and clay minerals (by centrifuge), both of which are unrelated to the source of the primary ash. The result is that there is a slight increase in percentage ( $93 \%$ and 98 $\%$ ) of the carbonate and clay fraction moving from the Russell to the Lebreton cores (Fig. 10A, Table 1). Within the bulk residue, there is no significant stratigraphic variation between the lower (RU 3) and upper shale (RU 5 and 6) samples in the Russell core (Fig. $10 \mathrm{~B})$, but there is, again, an increase in the finer-grained fraction moving towards the Lebreton core site (Fig. 10B).

The size of biotite grains shows an obvious lateral change between the two core sites. Biotite is more abundant $(<10 \%)$ and grains are much larger $(300-400 \mu \mathrm{m})$ in the Russell core compared to a lesser abundance $(<5 \%)$ and finer-grained fraction in the 
Figure 10. Grain size and type abundance. A) Frequency \% histograms of the carbonate and clay mineral fraction versus remainder of bulk sediment. B) Percentage of mud versus sand-size material. Both approaches demonstrate that the mud-size fraction increases toward the northwest, and the non-clay content decreases toward the northwest. In the Russell core, samples RU6 and RU5 are from the Lower bed, RU3 is from the lower bed; in the Billings Bridge core, BB10 is from the Upper bed. LEB. 15 is from the equivalent compacted shale. 


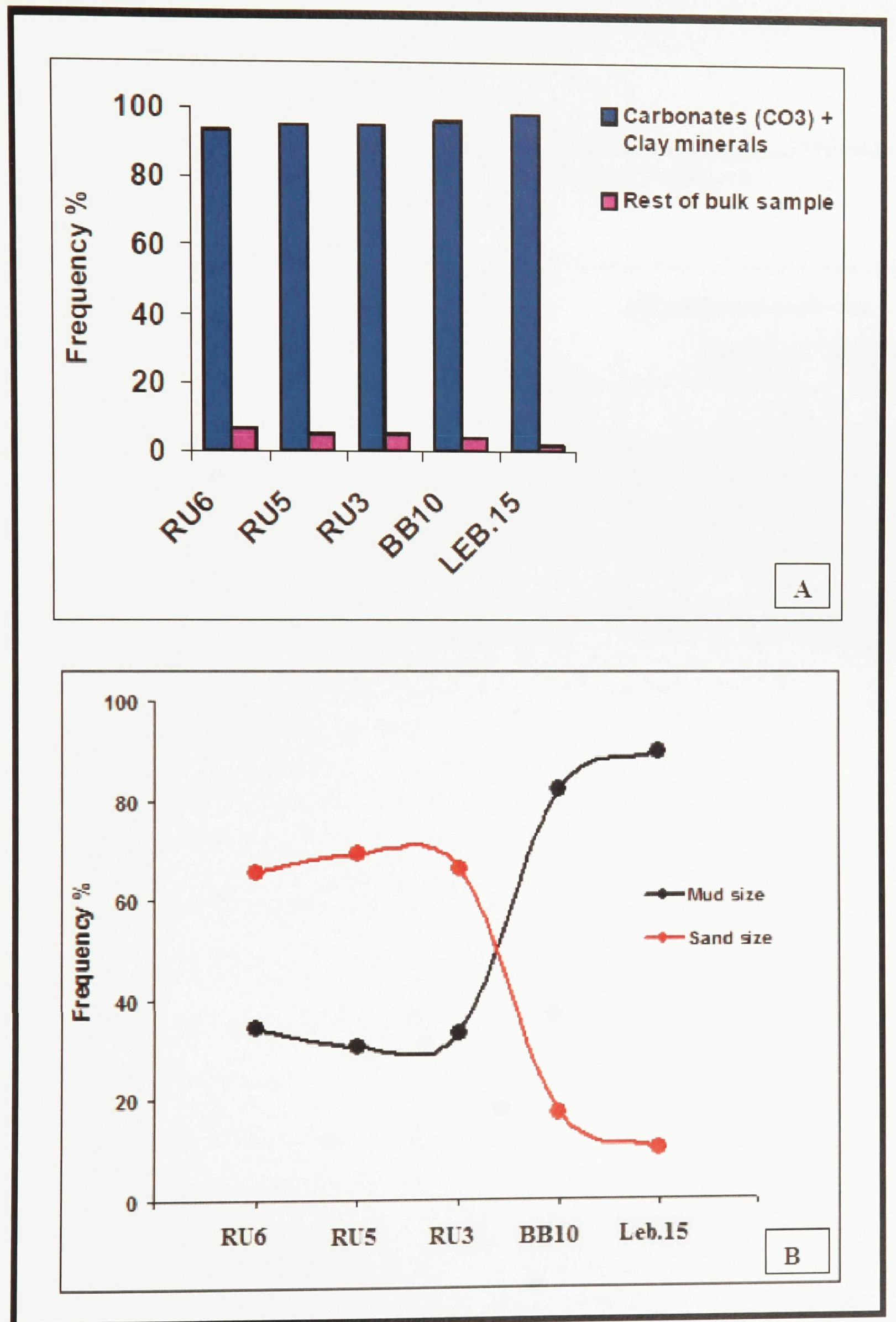


Table 1. Carbonate and clay mineral percentage and mineralogical grain size distribution of friable shale (Russell, Billings Bridge) and compacted shale (Lebreton).

\begin{tabular}{|c|c|c|c|c|c|c|c|c|c|c|c|}
\hline \multirow{3}{*}{$\begin{array}{l}\text { Ash } \\
\text { Core }\end{array}$} & \multirow{3}{*}{$\begin{array}{c}\text { Ash } \\
\text { Zone }\end{array}$} & \multirow{3}{*}{$\begin{array}{c}\text { Sample } \\
\#\end{array}$} & \multirow{3}{*}{$\begin{array}{l}\text { Total } \\
\text { Bulk } \\
\text { (gm) }\end{array}$} & \multicolumn{2}{|c|}{$\begin{array}{c}\text { Carbonate } \\
\text { material }(\mathrm{CO} 3)+ \\
\text { Clay minerals }\end{array}$} & \multicolumn{6}{|c|}{ Silt to fine sand-size residue } \\
\hline & & & & \multirow{2}{*}{$\begin{array}{l}\text { Quantity } \\
\text { (gm) }\end{array}$} & \multirow[t]{2}{*}{$(\%)$} & \multirow{2}{*}{$\begin{array}{c}\text { Quantity } \\
\text { (gm) }\end{array}$} & \multirow[t]{2}{*}{$(\%)$} & \multicolumn{2}{|c|}{$\begin{array}{l}\text { Mud-silt-size } \\
(<0.063 \mathrm{~mm})\end{array}$} & \multicolumn{2}{|c|}{$\begin{array}{l}\text { Fine sand-size } \\
(>0.063 \mathrm{~mm})\end{array}$} \\
\hline & & & & & & & & Q & $(\%)$ & Q & $(\%)$ \\
\hline \multirow[t]{3}{*}{ Russell } & \multirow[b]{2}{*}{ Lower } & RU6 & 1.02 & 0.95 & 93.14 & 0.07 & 6.86 & 0.024 & 2.35 & 0.046 & 4.51 \\
\hline & & RU5 & 0.915 & 0.869 & 94.97 & 0.046 & 5.03 & 0.014 & 1.53 & 0.032 & 3.5 \\
\hline & Upper & RU3 & 0.236 & 0.224 & 94.92 & 0.012 & 5.08 & 0.004 & 1.69 & 0.008 & 3.39 \\
\hline $\begin{array}{l}\text { Billings } \\
\text { Bridge }\end{array}$ & Upper & $\mathrm{BB} 10$ & 1.005 & 0.965 & 96.02 & 0.04 & 3.98 & 0.033 & 3.28 & 0.007 & 0.7 \\
\hline \multicolumn{2}{|c|}{ Lebreton } & LEB.15 & 0.106 & 0.104 & 98.11 & 0.002 & 1.89 & 0.0018 & 1.7 & 0.0002 & 0.19 \\
\hline
\end{tabular}


Billings Bridge core (50 to $75 \mu \mathrm{m}$; Fig. 11C). It remains uncertain if slight variations in biotite frequency among the lower and upper friable shales record stratigraphic variation given the potential larger error in measurement (related to sieving) compared to absolute differences in frequency. Examination of the equivalent dark-grey compacted shale within the Lebreton core reveals trace amounts of a subhedral dark brown mica, similar texturally to that found in the friable shales in the Billings and Russell cores, and of coarse silt size, similar to the mode in the Billings Bridge core.

Rounded silt- to sand-size $(<150$ microns) grains of clay, some with contained fragmental material, occur in both shale beds in both the Russell and Billings Bridge cores, but as with biotite, there is a NW-directed fining of these particles between core sites (Fig. 11D \& E). In the Russell core, the particles have elliptical to oval shapes with $\mathrm{L} / \mathrm{W}$ mean values of $1.64 \pm 0.24$ and $1.75 \pm 0.54$ for the upper and lower beds, respectively (Fig. 12). There is no stratigraphic significant difference in these values. Equivalent particles in the lower bed in the Billings Bridge core are predominantly elongate, with an $\mathrm{L} / \mathrm{W}$ mean value of $2.24 \pm 1.46$. There was insufficient material in the upper bed of the Billings core for a similar analysis. Thus, in addition to a NW fining in particle size, from Russell to Billings, there is also an increase in L/W ratios. A comparison of cumulative frequency plots (Fig. 12 and 13, Table 2, and Appendix A) demonstrate similar patterns.

In summary, there is a prominent decrease in grain size and abundance of mica moving northwest from the Russell core site, over $\sim 24 \mathrm{~km}$. There is a relatively abrupt drop in biotite abundance to trace amounts over a distance of $\sim 3 \mathrm{~km}$ between the 
Figure 11. Biotite abundance and grain size distribution. A and B) Different grain size, shape, and color of pseudohexagonal biotite. C) Decreasing grain size $(\mu)$ of biotite from SE (Russell Core) to NW (Billings Bridge). D and E) The change in the grain size of lithic fragments in Russell and Billings Bridge Cores, respectively. 

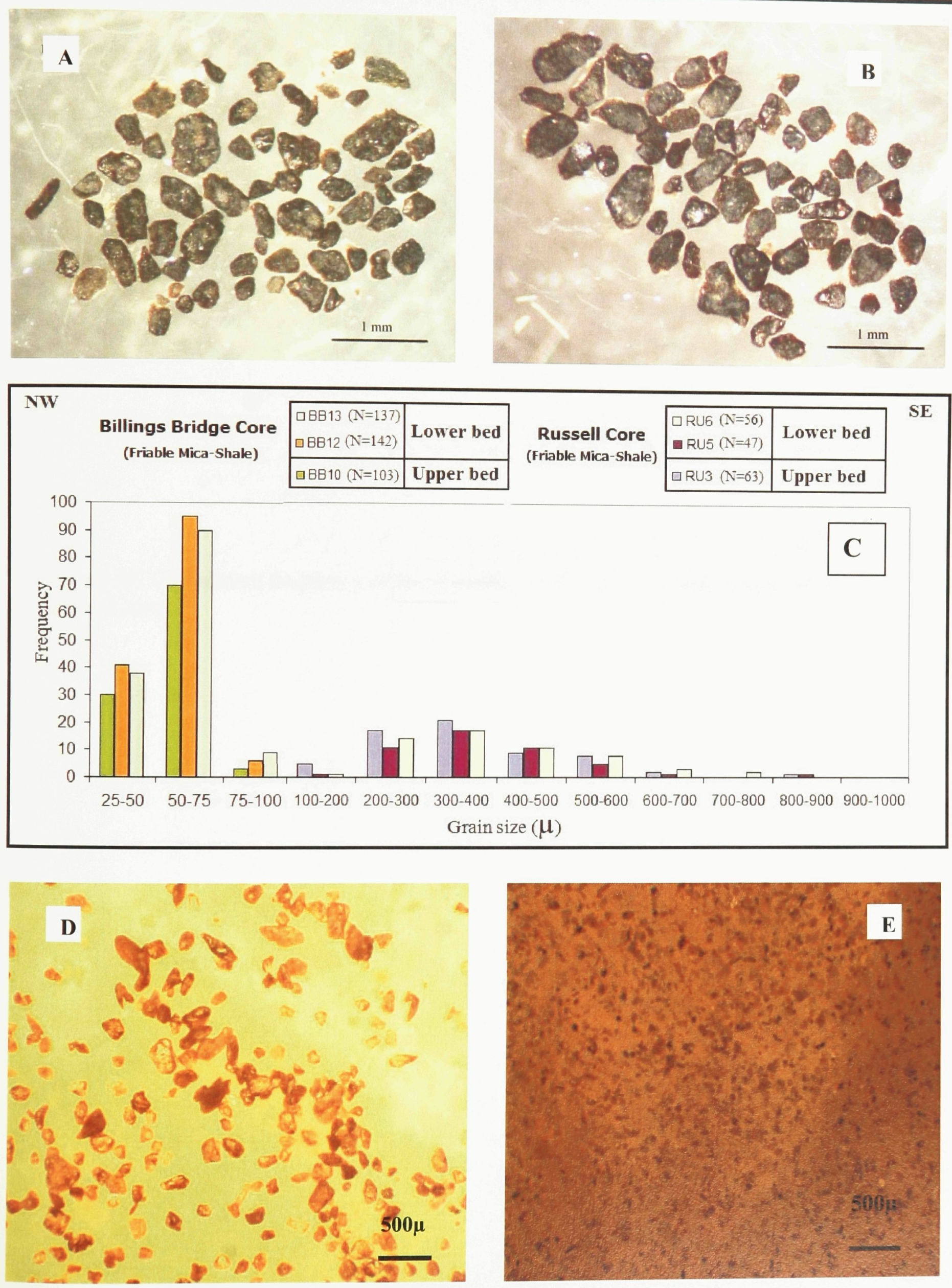
Figure 12. Cumulative frequency of length/width (L/W) ratios for lithic fragments in Russell core. 

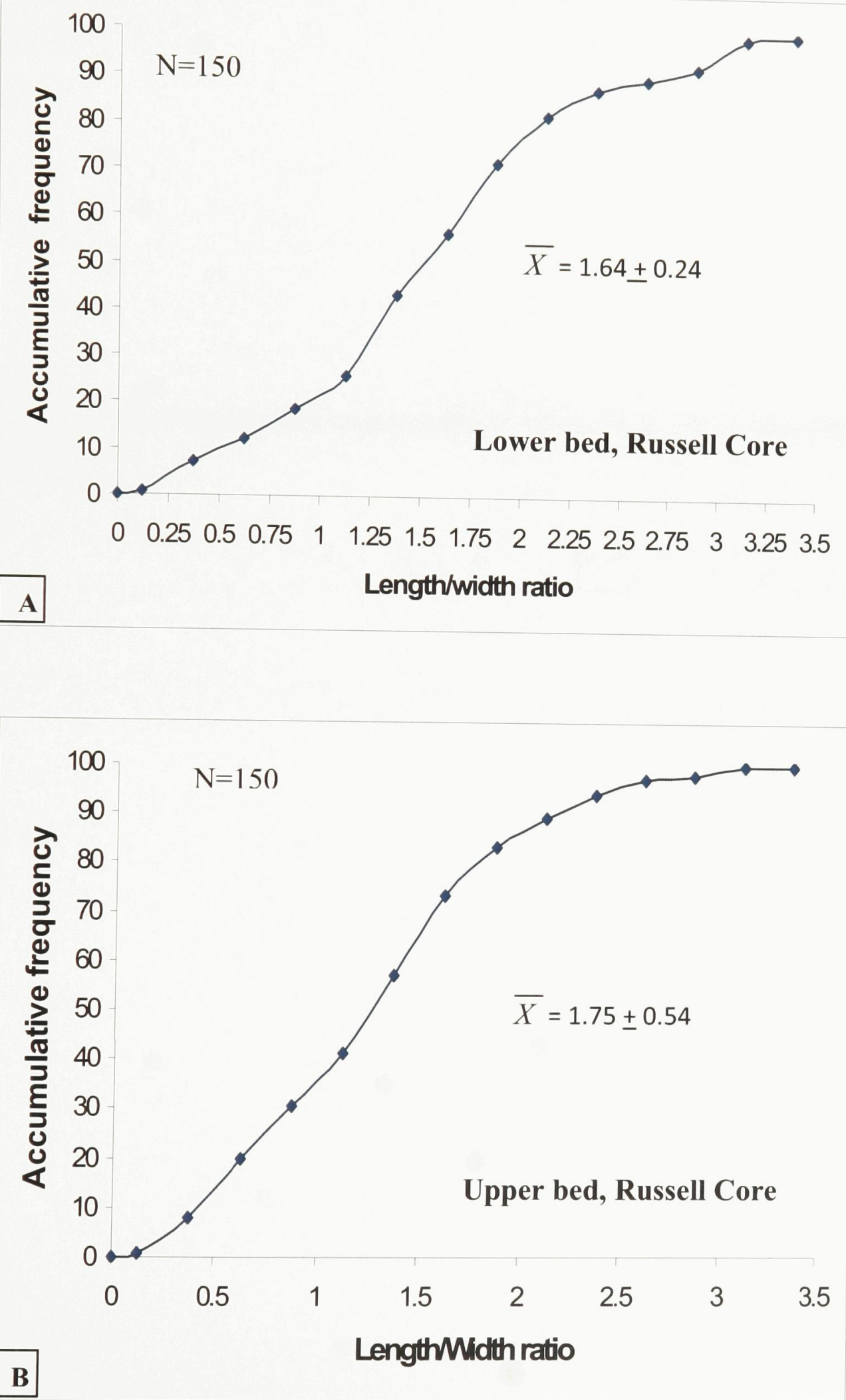
Figure 13. Cumulative frequency of length/width $(\mathrm{L} / \mathrm{W})$ ratio for lithic fragments in the Billings Bridge core. 


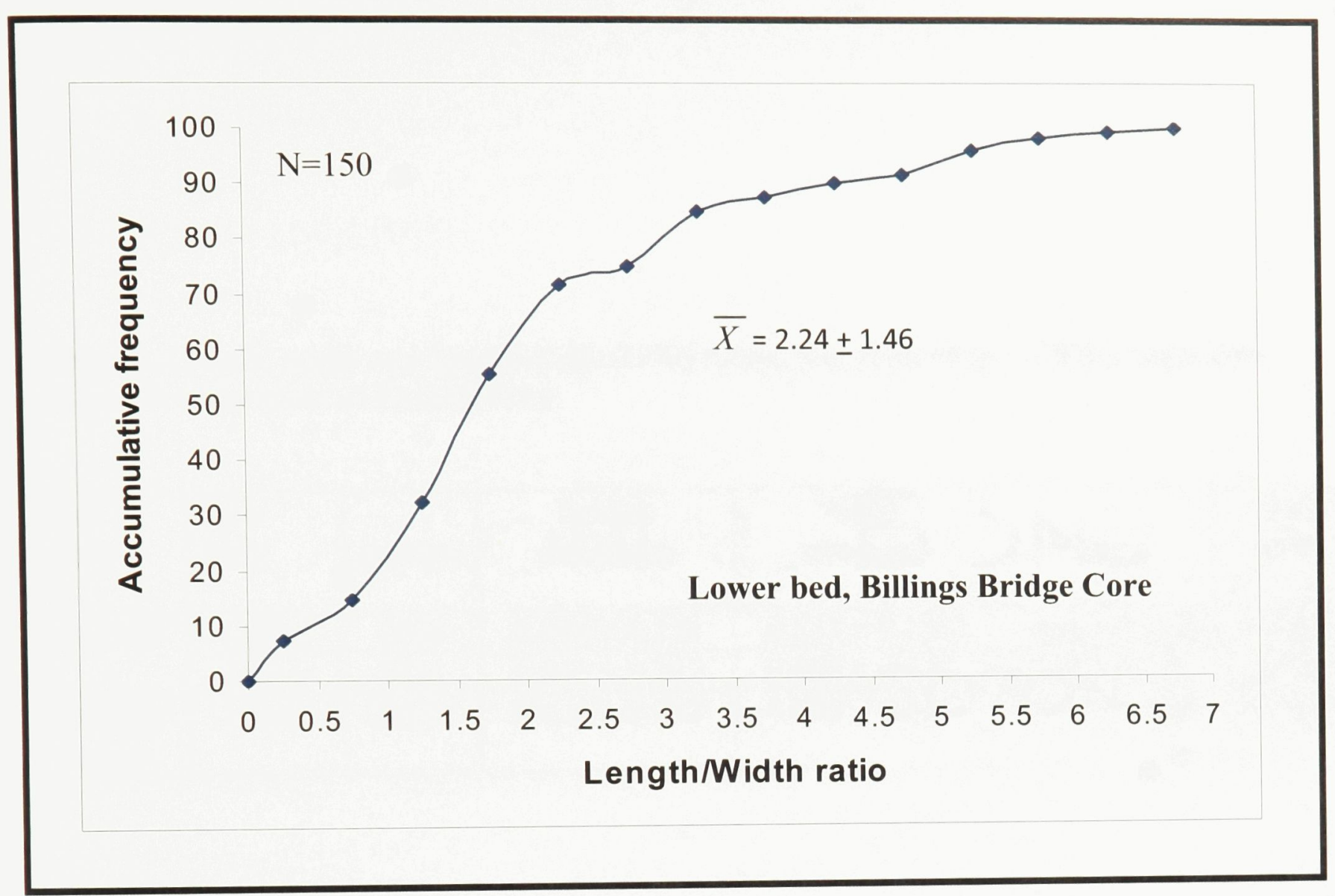


Table 2. Length, width and length/width (L/W) ratios, and grain shape of lithic fragments in friable mica-shale of Russell Core.

\begin{tabular}{|l|l|c|c|c|c|c|}
\hline $\begin{array}{l}\text { Ash } \\
\text { Core }\end{array}$ & $\begin{array}{l}\text { Ash } \\
\text { zone }\end{array}$ & Sample & $\begin{array}{c}\text { Length } \\
\text { (median) }\end{array}$ & $\begin{array}{c}\text { Width } \\
\text { (median) }\end{array}$ & L/W Ratio & $\begin{array}{c}\text { Grain } \\
\text { shape }\end{array}$ \\
\hline \multirow{2}{*}{ Russell } & Lower & RU6 & $0.946 \pm 0.238$ & $0.583 \pm 0.225$ & $1.641 \pm 0.238$ & Ellipse \\
\cline { 2 - 7 } & Upper & RU3 & $0.643 \pm 0.267$ & $0.362 \pm 0.042$ & $1.750 \pm 0.538$ & Oval \\
\hline $\begin{array}{c}\text { Billings } \\
\text { Bridge }\end{array}$ & Upper & BB10 & $0.175 \pm 0.082$ & $0.100 \pm 0.055$ & $2.241 \pm 1.460$ & Elongate \\
\hline
\end{tabular}


Billings Bridge and Lebreton core sites. No biotite was recovered from the Nepean site further supporting the northwest termination of biotite distribution.

\section{Interbedded carbonate}

Fossiliferous limestone bounds and lies interbedded with the friable shale beds in both the Russell and Billings Bridge cores (Fig. 5). The interbedded carbonate consists of blue-grey weathering bioclastic floatstone with a packstone to grainstone matrix. Thinsection analysis identifies fragments of crinoids, trilobites, brachiopods, and rare gastropods, as well as rare pellets. There is a prominent alignment of fossil material to bedding. The sediments are cemented by blocky intergranular calcite cement (Fig. 14A \& B). Using cathodoluminescence (CL), the intergranular cement has a deep red colour and dull luminescence.

\section{MINERALOGY OF FRIABLE SHALE}

Biotite: Biotite crystals are subhedral to euhedral (Fig. 15A-C), dark brown, and range from red to very dark brown in incident light, a change that might reflect the range in degree of alteration and magnetic intensity (Huff et. al, 1997). Biotite is pleochroic and some crystals contain rounded holes (Fig. 15A), likely where an inclusion has been removed (possibly during preparation). Back-scattered scanning electron microscopy of subhedral to rounded biotite clasts reveals that remnant lamellae of unaltered biotite within an illitic host or a mixture of illite and chlorite (Figs. 15E and F, Table 3). Biotite is not well represented in X-ray diffraction spectra (Fig. 16) as supported by the extensive alteration illustrated by SEM. 
Figure 14. Photomicrograph of skeletal floatstone with grainstone to packstone matrix of carbonates rocks associated with the friable shale. A, Russell Core, (RU4), B, Billings Bridge core, (BB11). 

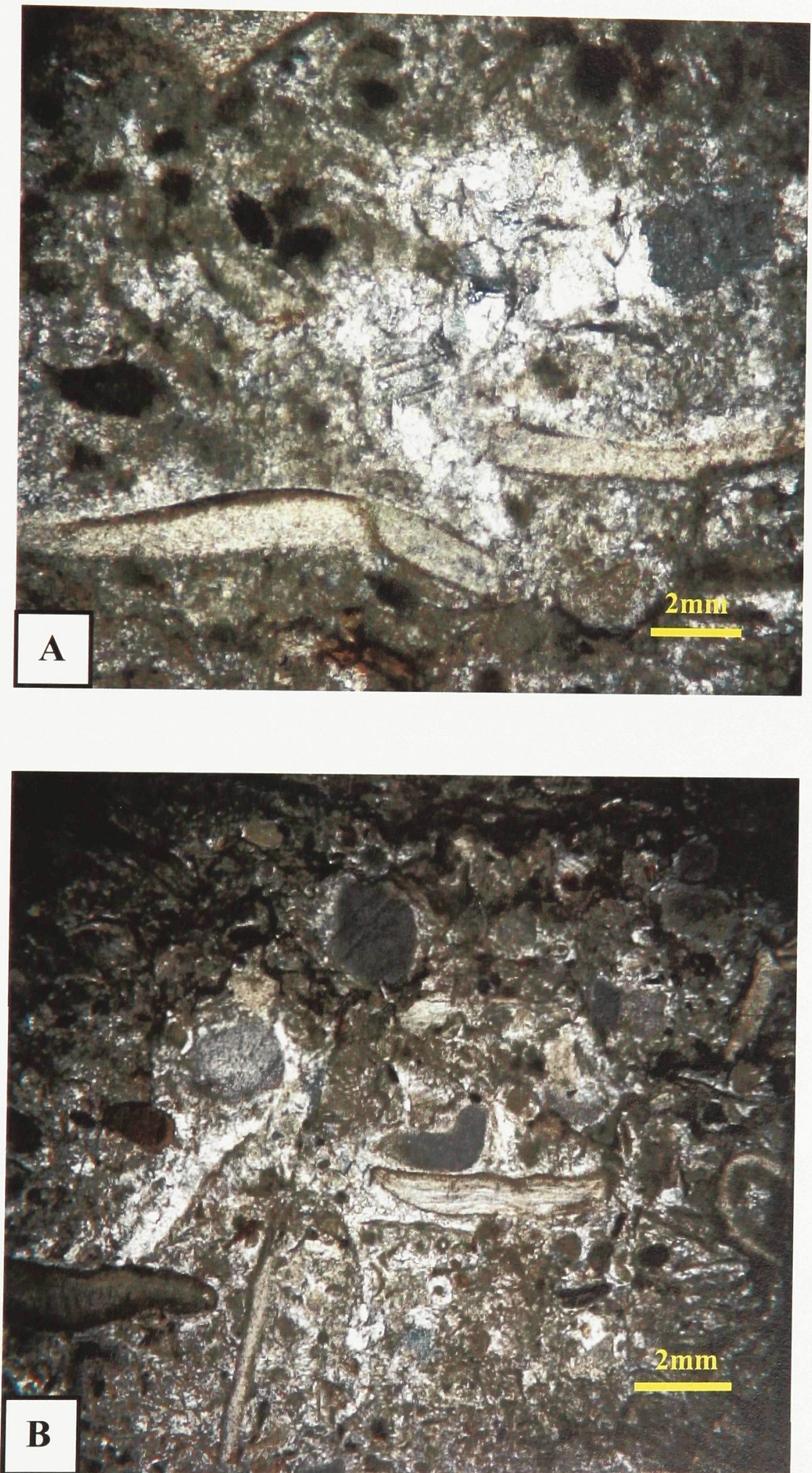
Figure 15. SEM photomicrographs of friable shale components. A), B), and C) are secondary electron photomigraphs showing subeuhedral and pseudohexagonal habits of biotite, with a zircon inclusion in $(\mathrm{C})$ below pink squares, which are raster areas over which analysis confirmed biotite composition. D) Back-scattered electron image of lithic fragment showing subrounded to angular fragments of quartz (qtz) and potassic feldspar (ksp), along with framboidal pyrite (py), illite clay (ill), and calcium carbonate (cc). E) and F) Back-scattered electron photomicrograph showing lamellae of relict biotite (bright) within a matrix of chlorite and illite, respectively. 


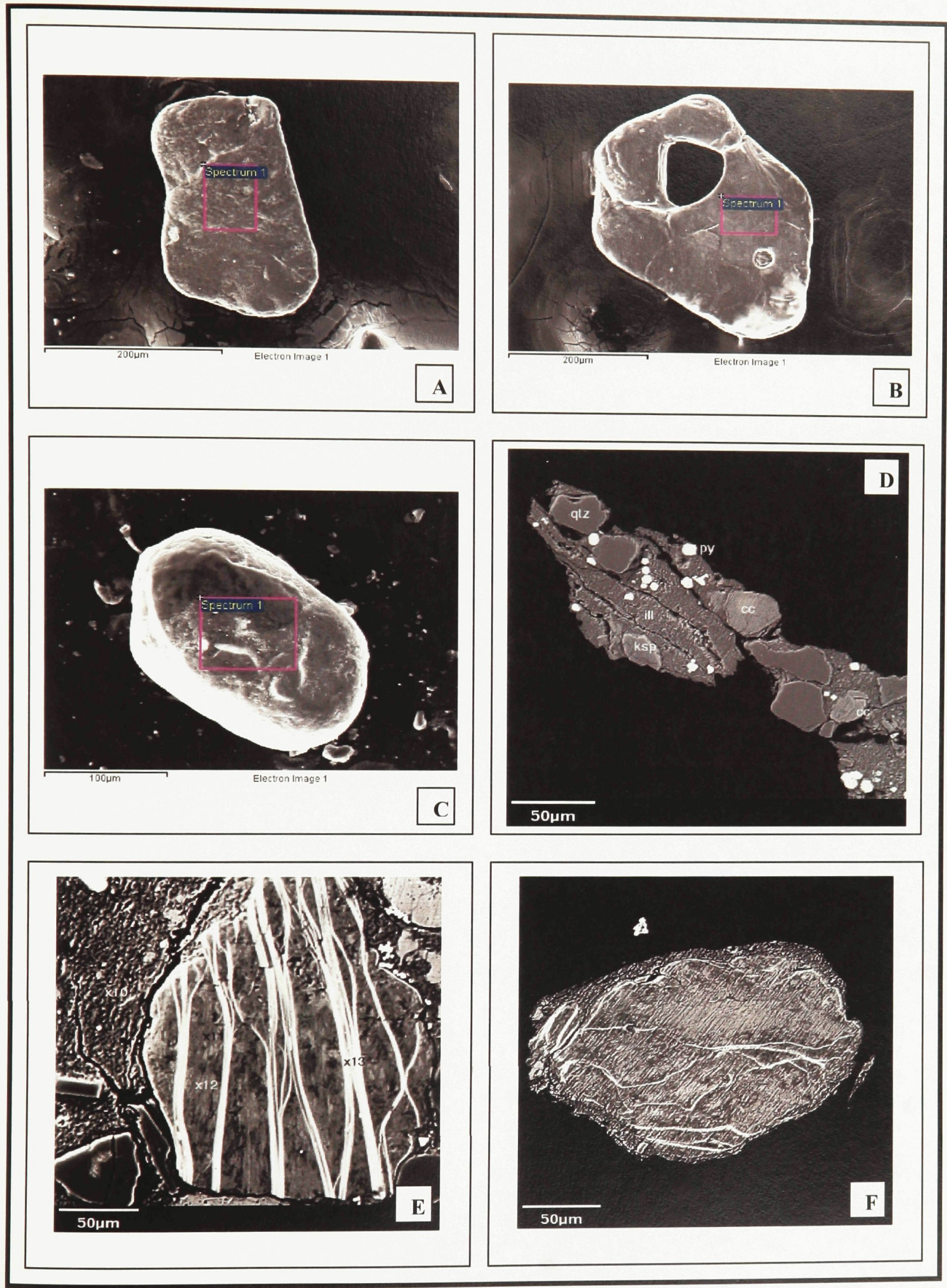


Figure 16. X-ray diffractograms of oriented samples. A) bulk shale, B) untreated clay size fraction. 

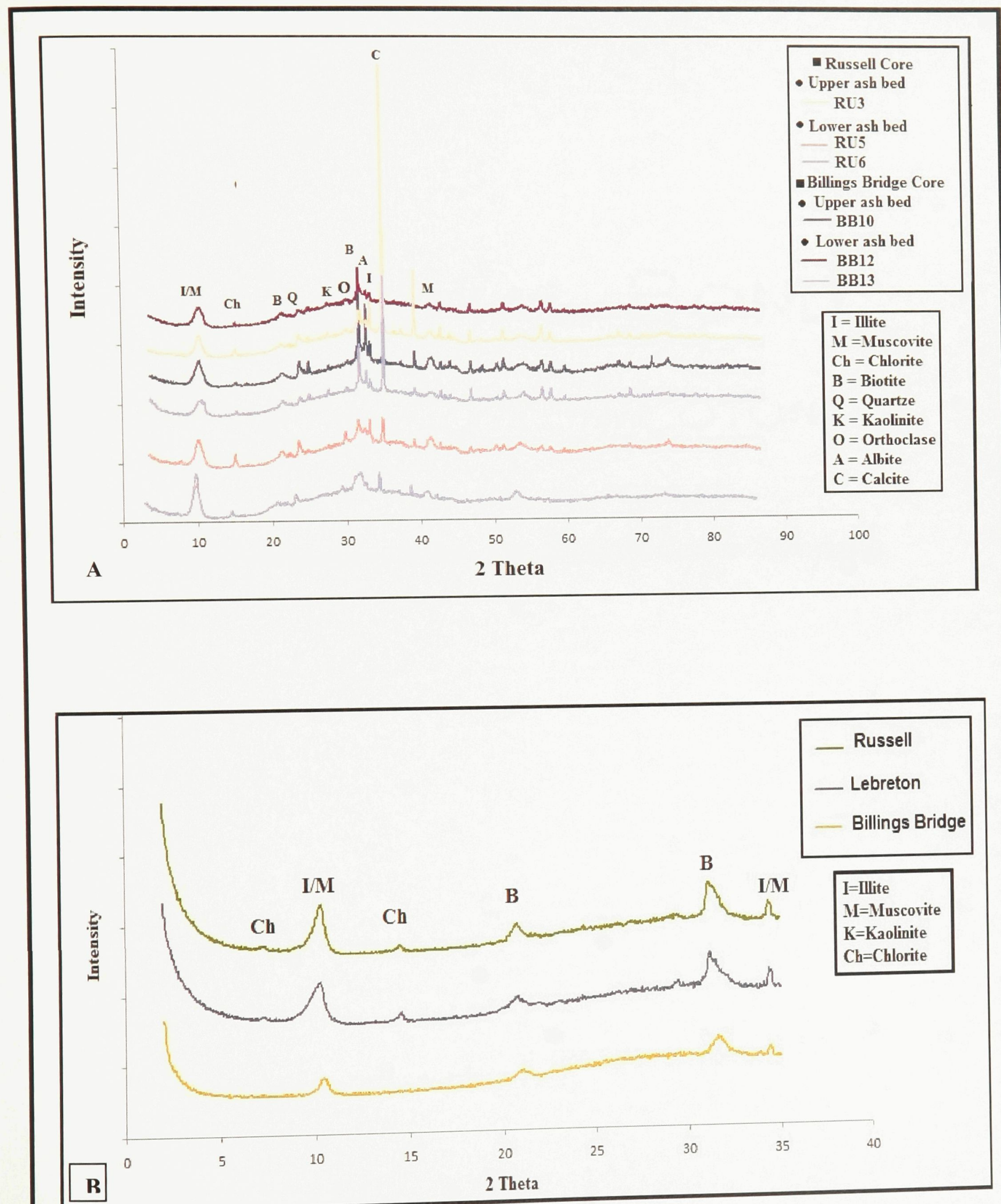
Table 3. Elemental composition of biotite, illite and chlorite in friable shale samples. 


\begin{tabular}{|c|c|c|c|c|c|c|c|c|c|c|c|c|}
\hline \multirow{3}{*}{ Oxides } & \multicolumn{6}{|c|}{ Verulam Ash-RUSSELL CORE } & \multicolumn{6}{|c|}{ Verulam Ash-BILLINGS BRIDGE CORE } \\
\hline & \multicolumn{3}{|c|}{$\begin{array}{l}\text { UPPER } \\
\text { (RU3) }\end{array}$} & \multicolumn{3}{|c|}{$\begin{array}{c}\text { LOWER } \\
(\text { RU6-RU5)* }\end{array}$} & \multicolumn{3}{|c|}{$\begin{array}{l}\text { UPPER } \\
\text { (BB10) }\end{array}$} & \multicolumn{3}{|c|}{$\begin{array}{c}\text { LOWER } \\
(\text { BB12-BB13)* }\end{array}$} \\
\hline & $\begin{array}{l}\text { Biotite } \\
\mathrm{N}=3\end{array}$ & $\begin{array}{l}\text { Illite } \\
\mathrm{N}=2\end{array}$ & $\begin{array}{l}\text { Chlorite } \\
\mathrm{N}=3\end{array}$ & $\begin{array}{l}\text { Biotite } \\
\mathrm{N}=3\end{array}$ & $\begin{array}{l}\text { Illite } \\
\mathrm{N}=6\end{array}$ & $\begin{array}{l}\text { Chlorite } \\
\mathrm{N}=10\end{array}$ & $\begin{array}{l}\text { Biotite } \\
\mathrm{N}=1\end{array}$ & \begin{tabular}{|l} 
Illite \\
$\mathrm{N}=2$
\end{tabular} & \begin{tabular}{|l|} 
Chlorite \\
$\mathrm{N}=2$
\end{tabular} & $\begin{array}{l}\text { Biotite } \\
\mathrm{N}=3\end{array}$ & $\begin{array}{l}\text { Illite } \\
\mathrm{N}=2\end{array}$ & $\begin{array}{l}\text { Chlorite } \\
\mathrm{N}=4\end{array}$ \\
\hline $\mathrm{SiO} 2$ & $\begin{array}{l}35.26 \\
(1.05) \\
\end{array}$ & $\begin{array}{l}55.49 \\
(4.51) \\
\end{array}$ & $\begin{array}{l}33.48 \\
(2.11) \\
\end{array}$ & $\begin{array}{l}35.70 \\
(0.24) \\
\end{array}$ & $\begin{array}{l}53.72 \\
(4.29) \\
\end{array}$ & $\begin{array}{l}33.07 \\
(0.33) \\
\end{array}$ & 34.24 & $\begin{array}{l}55.85 \\
(1.4) \\
\end{array}$ & $\begin{array}{l}37.37 \\
(1.87) \\
\end{array}$ & $\begin{array}{r}34.95 \\
(1.2) \\
\end{array}$ & $\begin{array}{r}56.73 \\
(3.17) \\
\end{array}$ & $\begin{array}{l}37.08 \\
(1.26) \\
\end{array}$ \\
\hline $\mathrm{Al} 2 \mathrm{O3}$ & $\begin{array}{l}15.94 \\
(1.17) \\
\end{array}$ & $\begin{array}{l}26.78 \\
(1.40) \\
\end{array}$ & $\begin{array}{l}25.72 \\
(1.39) \\
\end{array}$ & $\begin{array}{c}15.5 \\
(2.04) \\
\end{array}$ & $\begin{array}{l}27.25 \\
(2.08) \\
\end{array}$ & $\begin{array}{l}25.52 \\
(0.99)\end{array}$ & 16 & $\begin{array}{l}24.46 \\
(2.24)\end{array}$ & $\begin{array}{c}26.34 \\
(0.28)\end{array}$ & $\begin{array}{l}16.21 \\
(0.86) \\
\end{array}$ & $\begin{array}{l}26.08 \\
(2.62)\end{array}$ & $\begin{array}{l}26.67 \\
(1.47)\end{array}$ \\
\hline $\mathrm{TiO} 2$ & $\begin{array}{c}4.57 \\
(0.43) \\
\end{array}$ & $\begin{array}{c}0.19 \\
(0.03) \\
\end{array}$ & $\begin{array}{c}0.03 \\
(0.04) \\
\end{array}$ & $\begin{array}{c}4.49 \\
(0.48) \\
\end{array}$ & $\begin{array}{c}0.26 \\
(0.27) \\
\end{array}$ & $\begin{array}{l}0.052 \\
(0.04) \\
\end{array}$ & 3.94 & $\begin{array}{c}0.81 \\
(0.60) \\
\end{array}$ & $\begin{array}{c}0.05 \\
(0.01) \\
\end{array}$ & $\begin{array}{c}4.73 \\
(1.05) \\
\end{array}$ & $\begin{array}{c}(2.02) \\
0.68 \\
(0.57)\end{array}$ & $\begin{array}{c}(1.7) \\
0.01 \\
(0.01)\end{array}$ \\
\hline $\mathrm{Cr} 2 \mathrm{O} 3$ & 0.0 & 0.0 & $\begin{array}{c}0.02 \\
(0.02) \\
\end{array}$ & $\begin{array}{c}0.02 \\
(0.02) \\
\end{array}$ & $\begin{array}{c}0.01 \\
(0.01) \\
\end{array}$ & $\begin{array}{c}0.01 \\
(0.01) \\
\end{array}$ & 0.0 & $\begin{array}{c}0.01 \\
(0.01)\end{array}$ & 0.0 & $\begin{array}{c}0.01 \\
(0.02)\end{array}$ & $\begin{array}{c}0.01 \\
(0.01) \\
\end{array}$ & $\begin{array}{c}0.01 \\
(0.01)\end{array}$ \\
\hline $\mathrm{FeO}$ & $\begin{array}{l}22.10 \\
(0.95) \\
\end{array}$ & $\begin{array}{c}1.46 \\
(0.29) \\
\end{array}$ & $\begin{array}{r}14.41 \\
(2.54) \\
\end{array}$ & $\begin{array}{l}21.47 \\
(1.24) \\
\end{array}$ & $\begin{array}{c}1.76 \\
(2.08) \\
\end{array}$ & $\begin{array}{l}14.78 \\
(1.14) \\
\end{array}$ & 22.06 & $\begin{array}{c}1.36 \\
(0.33) \\
\end{array}$ & $\begin{array}{l}12.28 \\
(1.17) \\
\end{array}$ & $\begin{array}{r}19.75 \\
(1.46) \\
\end{array}$ & $\begin{array}{c}2.00 \\
(0.76) \\
\end{array}$ & $\begin{array}{c}14.03 \\
(0.22) \\
\end{array}$ \\
\hline $\mathrm{MgO}$ & $\begin{array}{c}9.92 \\
(0.74) \\
\end{array}$ & $\begin{array}{c}3.69 \\
(0.13) \\
\end{array}$ & $\begin{array}{r}13.18 \\
(0.96) \\
\end{array}$ & $\begin{array}{c}9.49 \\
(0.93) \\
\end{array}$ & $\begin{array}{c}3.68 \\
(1.37) \\
\end{array}$ & $\begin{array}{c}0.01 \\
(0.01)\end{array}$ & 10.34 & $\begin{array}{c}3.32 \\
(0.13) \\
\end{array}$ & $\begin{array}{l}0.025 \\
(0.04) \\
\end{array}$ & $\begin{array}{l}9.80 \\
(0.8) \\
\end{array}$ & $\begin{array}{c}3.62 \\
(0.41) \\
\end{array}$ & $\begin{array}{l}10.525 \\
(2.76) \\
\end{array}$ \\
\hline $\mathrm{MnO}$ & $\begin{array}{c}0.14 \\
(0.03) \\
\end{array}$ & 0.0 & $\begin{array}{c}0.04 \\
(0.04) \\
\end{array}$ & $\begin{array}{c}0.17 \\
(0.03) \\
\end{array}$ & $\begin{array}{c}0.01 \\
(0.01) \\
\end{array}$ & $\begin{array}{l}13.40 \\
(0.47) \\
\end{array}$ & 0.13 & $\begin{array}{c}0.01 \\
(0.02) \\
\end{array}$ & $\begin{array}{l}11.66 \\
(1.20)\end{array}$ & $\begin{array}{c}0.09 \\
(0.08) \\
\end{array}$ & $\begin{array}{c}0.02 \\
(0.01)\end{array}$ & $\begin{array}{c}0.02 \\
(0.01)\end{array}$ \\
\hline $\mathrm{NiO}$ & 0.0 & 0.0 & 0 & 0.0 & 0.0 & 0.0 & 0.0 & 0.0 & 0.0 & 0.0 & 0.0 & 0.0 \\
\hline K2O & $\begin{array}{c}6.74 \\
(1.02) \\
\end{array}$ & $\begin{array}{c}6.44 \\
(0.17) \\
\end{array}$ & $\begin{array}{c}1.12 \\
(0.57) \\
\end{array}$ & $\begin{array}{c}7.42 \\
(1.12)\end{array}$ & $\begin{array}{c}6.71 \\
(0.86) \\
\end{array}$ & $\begin{array}{c}0.99 \\
(0.25) \\
\end{array}$ & 6.16 & $\begin{array}{c}6.62 \\
(0.17) \\
\end{array}$ & $\begin{array}{c}0.12 \\
(0.01)\end{array}$ & $\begin{array}{r}6.19 \\
(1.07) \\
\end{array}$ & $\begin{array}{c}6.72 \\
(0.19) \\
\end{array}$ & $\begin{array}{c}1.81 \\
(0.13)\end{array}$ \\
\hline $\mathrm{CaO}$ & $\begin{array}{c}0.04 \\
(0.07)\end{array}$ & $\begin{array}{c}1.74 \\
(0.61)\end{array}$ & $\begin{array}{c}0.13 \\
(0.08) \\
\end{array}$ & $\begin{array}{c}0.07 \\
(0.03)\end{array}$ & $\begin{array}{c}1.47 \\
(2.06)\end{array}$ & $\begin{array}{c}0.12 \\
(0.01)\end{array}$ & 0.0 & $\begin{array}{c}1.86 \\
(1.29) \\
\end{array}$ & $\begin{array}{c}2.20 \\
(0.77)\end{array}$ & $\begin{array}{c}0.04 \\
(0.04)\end{array}$ & $\begin{array}{c}0.92 \\
(0.48)\end{array}$ & $\begin{array}{c}0.16 \\
(0.04)\end{array}$ \\
\hline $\mathrm{Na2O}$ & $\begin{array}{c}0.22 \\
(0.09)\end{array}$ & $\begin{array}{c}0.43 \\
(0.03)\end{array}$ & 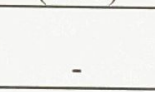 & $\begin{array}{c}0.26 \\
(0.06)\end{array}$ & $\begin{array}{c}0.35 \\
(0.09)\end{array}$ & - & 0.19 & $\begin{array}{c}0.52 \\
(0.33)\end{array}$ & - & $\begin{array}{c}0.21 \\
(0.06) \\
\end{array}$ & $\begin{array}{c}0.34 \\
(0.05)\end{array}$ & - \\
\hline $\mathrm{BaO}$ & $\begin{array}{c}0.56 \\
(0.06) \\
\end{array}$ & $\begin{array}{c}0.01 \\
(0.02)\end{array}$ & - & $\begin{array}{c}0.36 \\
(0.17) \\
\end{array}$ & $\begin{array}{c}0.02 \\
(0.02)\end{array}$ & - & 0.33 & $\begin{array}{c}0.01 \\
(0.01)\end{array}$ & - & $\begin{array}{c}0.38 \\
(0.14)\end{array}$ & $\begin{array}{c}0.02 \\
(0.01)\end{array}$ & - \\
\hline F & $\begin{array}{c}0.54 \\
(0.11) \\
\end{array}$ & $\begin{array}{c}1.19 \\
(0.16) \\
\end{array}$ & $\begin{array}{c}0.08 \\
(0.04)\end{array}$ & $\begin{array}{c}0.46 \\
(0.06)\end{array}$ & $\begin{array}{c}0.98 \\
(0.38)\end{array}$ & $\begin{array}{c}0.08 \\
(0.03)\end{array}$ & 0.59 & $\begin{array}{c}1.16 \\
(0.16)\end{array}$ & 0.07 & $\begin{array}{c}0.68 \\
(0.07)\end{array}$ & $\begin{array}{c}0.88 \\
(0.13) \\
\end{array}$ & 0.04 \\
\hline $\mathrm{Cl}$ & $\begin{array}{c}0.13 \\
(0.01) \\
\end{array}$ & $\begin{array}{c}0.03 \\
(0.01) \\
\end{array}$ & $\begin{array}{c}0.44 \\
(0.40)\end{array}$ & $\begin{array}{c}0.10 \\
(0.01)\end{array}$ & $\begin{array}{c}0.07 \\
(0.08)\end{array}$ & $\begin{array}{c}0.43 \\
(0.14) \\
\end{array}$ & 0.07 & $\begin{array}{c}0.06 \\
(0.33)\end{array}$ & 0.36 & $\begin{array}{c}0.1 \\
(0.02)\end{array}$ & $\begin{array}{c}0.04 \\
(0.01)\end{array}$ & 0.49 \\
\hline TOTAL & 96.16 & 97.48 & 88.65 & 95.51 & 96.29 & 88.46 & 94.05 & 96.04 & 90.45 & 93.10 & 98.02 & 90.82 \\
\hline TOT-O & 95.91 & 96.95 & 88.42 & 95.29 & 95.86 & 88.26 & 93.56 & 95.54 & 90.28 & 92.85 & 97.64 & 90.6 \\
\hline $\mathrm{Si}$ & 5.40 & 7.10 & 6.46 & 5.49 & 6.98 & 6.41 & 5.614 & 7.27 & 6.95 & 5.44 & 7.20 & 6.92 \\
\hline $\mathrm{Al}$ & 2.60 & 0.90 & 1.54 & 2.51 & 1.02 & 1.59 & 2.386 & 0.73 & 1.05 & 2.56 & 0.80 & 1.08 \\
\hline SUBT & 8 & 8 & 8 & 8 & 8 & 8 & 8 & 8 & 8 & 8 & 8 & 8 \\
\hline $\mathrm{Al}$ & 0.27 & 3.15 & 4.31 & 0.30 & 3.15 & 4.24 & 0.444 & 3.02 & 4.72 & 0.42 & 3.11 & 4.78 \\
\hline $\mathrm{Ti}$ & 0.53 & 0.02 & 0.004 & 0.52 & 0.02 & 0.01 & 0.49 & 0.079 & 0.007 & 0.56 & 0.06 & 0.00 \\
\hline $\mathrm{Cr}$ & 0 & 0 & 0.003 & 0.002 & 0.001 & 0.00 & 0.007 & 0.001 & 0.0 & 0.004 & 0.00 & 0.001 \\
\hline $\mathrm{Fe}$ & 2.83 & 0.16 & 2.33 & 2.76 & 0.20 & 2.40 & 2.634 & 0.15 & 1.91 & 2.57 & 0.21 & 2.19 \\
\hline $\mathrm{Mg}$ & 2.26 & 0.71 & 0.01 & 2.17 & 0.72 & 3.8 & 2.032 & 0.64 & 3.23 & 2.27 & 0.67 & 0.00 \\
\hline Mn & 0.02 & 0 & 3.8 & 0.02 & 0.001 & 0.00 & 0.024 & 0.00 & 0.0 & 0.01 & 0.00 & 2.93 \\
\hline $\mathbf{K}$ & - & - & 0.27 & - & - & 0.24 & - & - & 0.52 & - & - & 0.43 \\
\hline $\mathrm{Ca}$ & - & - & 0.03 & - & - & 0.03 & - & - & 0.02 & - & - & 0.03 \\
\hline $\mathrm{Ni}$ & 0 & 0 & 0 & 0 & 0 & 0 & 0 & 0 & 0 & 0 & 0 & 0 \\
\hline SUBT & 5.91 & 4.03 & 10.76 & 5.78 & 4.1 & 10.79 & 5.631 & 3.89 & 10.41 & 5.84 & 4.07 & 10.36 \\
\hline $\mathrm{K}$ & 1.32 & 1.06 & - & 1.46 & 1.11 & - & 1.509 & 1.11 & - & 1.23 & 1.10 & - \\
\hline $\mathrm{Ca}$ & 0.01 & 0.24 & - & 0.01 & 0.21 & - & 0.014 & 0.26 & - & 0.01 & 0.12 & - \\
\hline $\mathrm{Na}$ & 0.07 & 0.11 & - & 0.08 & 0.09 & - & 0.089 & 0.13 & - & 0.06 & 0.08 & - \\
\hline $\mathrm{Ba}$ & 0.034 & 0.00 & - & 0.02 & 0.00 & - & 0.032 & 0.00 & - & 0.02 & 0.00 & - \\
\hline SUBT & 1.42 & 1.41 & - & 1.57 & 1.41 & - & 1.644 & 1.50 & - & 1.32 & 1.30 & - \\
\hline $\mathbf{F}$ & 0.26 & 0.48 & 0.03 & 0.23 & 0.40 & 0.03 & 0.366 & 0.48 & 0.02 & 0.33 & 0.35 & 0.01 \\
\hline $\mathrm{Cl}$ & 0.034 & 0.01 & 0.27 & 0.03 & 0.01 & 0.26 & 0.024 & 0.01 & 0.21 & 0.03 & 0.01 & 0.29 \\
\hline SUBT & 0.294 & 0.49 & 0.30 & 0.25 & 0.41 & 0.29 & 0.390 & 0.49 & 0.23 & 0.36 & 0.36 & 0.30 \\
\hline
\end{tabular}




\section{Biotite:}

1. Russell Core, Upper Ash (RU3):

$\mathrm{K}_{1.52}\left(\mathrm{Mg}, \mathrm{Fe}^{+2}\right)_{5.43}\left(\mathrm{Fe}^{+3}, \mathrm{Al}, \mathrm{Ti}\right)_{0.77}\left[\mathrm{Si}_{5.48} \mathrm{Al}_{2.52} \mathrm{O}_{20}\right]\left[\left(\mathrm{F}_{0.24}, \mathrm{Cl}_{0.036}\right)(\mathrm{OH})_{4}\right] \ldots 1$ $\mathrm{K}_{1.58}\left(\mathrm{Mg}, \mathrm{Fe}^{+2}\right)_{5.04}\left(\mathrm{Fe}^{+3}, \mathrm{Al}, \mathrm{Ti}\right)_{0.78}\left[\mathrm{Si}_{5.45} \mathrm{Al}_{2.55} \mathrm{O}_{20}\right]\left[\left(\mathrm{F}_{0.23}, \mathrm{Cl}_{0.034}\right)(\mathrm{OH})_{4}\right] \ldots 2$ $\mathrm{K}_{1.70}\left(\mathrm{Mg}, \mathrm{Fe}^{+2}\right)_{5.19}\left(\mathrm{Fe}^{+3}, \mathrm{Al}, \mathrm{Ti}\right)_{0.88}\left[\mathrm{Si}_{5.26} \mathrm{Al}_{2.74} \mathrm{O}_{20}\right]\left[\left(\mathrm{F}_{0.22}, \mathrm{Cl}_{0.032}\right)(\mathrm{OH})_{4}\right] \ldots 3$

The average chemical formula is:

$\mathrm{K}_{1.60}\left(\mathrm{Mg}, \mathrm{Fe}^{+2}\right)_{5.22}\left(\mathrm{Fe}^{+3}, \mathrm{Al}, \mathrm{Ti}\right)_{0.81}\left[\mathrm{Si}_{5.40} \mathrm{Al}_{2.60} \mathrm{O}_{20}\right]\left[\left(\mathrm{F}_{0.242} \mathrm{Cl}_{0.034}\right)(\mathrm{OH})_{4}\right]$ 4

2. Russell Core, Lower Ash (RU6):

$\mathrm{K}_{1.77}\left(\mathrm{Mg}, \mathrm{Fe}^{+2}\right)_{4.93}\left(\mathrm{Fe}^{+3}, \mathrm{Al}, \mathrm{Ti}\right)_{0.71}\left[\mathrm{Si}_{5.56} \mathrm{Al}_{2.44} \mathrm{O}_{20}\right]\left[\left(\mathrm{F}_{0.25}, \mathrm{Cl}_{0.024}\right)(\mathrm{OH})_{4}\right] \ldots 5$

$\mathrm{K}_{1.67}\left(\mathrm{Mg}, \mathrm{Fe}^{+2}\right)_{4.97}\left(\mathrm{Fe}^{+3}, \mathrm{Al}, \mathrm{Ti}\right)_{0.74}\left[\mathrm{Si}_{5.54} \mathrm{Al}_{2.46} \mathrm{O}_{20}\right]\left[\left(\mathrm{F}_{0.23}, \mathrm{Cl}_{0.028}\right)(\mathrm{OH})_{4}\right] \ldots 6$

$\mathrm{K}_{1.64}\left(\mathrm{Mg}, \mathrm{Fe}^{+2}\right)_{4.67}\left(\mathrm{Fe}^{+3}, \mathrm{Al}, \mathrm{Ti}\right)_{0.88}\left[\mathrm{Si}_{5.61} \mathrm{Al}_{2.39} \mathrm{O}_{20}\right]\left[\left(\mathrm{F}_{0.37}, \mathrm{Cl}_{0.024}\right)(\mathrm{OH})_{4}\right] \ldots 7$

The average chemical formula is:

$\mathrm{K}_{1.71}\left(\mathrm{Mg}, \mathrm{Fe}^{+2}\right)_{4.86}\left(\mathrm{Fe}^{+3}, \mathrm{Al}, \mathrm{Ti}\right)_{0.78}\left[\mathrm{Si}_{5.57} \mathrm{Al}_{2.43} \mathrm{O}_{20}\right]\left[\left(\mathrm{F}_{0.28} \mathrm{Cl}_{0.025}\right)(\mathrm{OH})_{4}\right] \ldots \ldots \ldots \ldots . . .8$

3. Billings Bridge core, Upper Ash Bed (BB10):

$\mathrm{K}_{1.31}\left(\mathrm{Mg}, \mathrm{Fe}^{+2}\right)_{5.29}\left(\mathrm{Fe}^{+3}, \mathrm{Al}, \mathrm{Ti}\right)_{0.75}\left[\mathrm{Si}_{5.35} \mathrm{Al}_{2.65} \mathrm{O}_{20}\right]\left[\left(\mathrm{F}_{0.29}, \mathrm{Cl}_{\mathbf{0 . 0 2 0}}\right)(\mathrm{OH})_{4}\right] \ldots 9$

4. Billings Bridge core, Lower Ash (BB13 and BB13)

$\mathrm{K}_{1.23}\left(\mathrm{Mg}, \mathrm{Fe}^{+2}\right)_{4.70}\left(\mathrm{Fe}^{+3}, \mathrm{Al}, \mathrm{Ti}\right)_{1.16}\left[\mathrm{Si}_{5.48} \mathrm{Al}_{2.52} \mathrm{O}_{20}\right]\left[\left(\mathrm{F}_{0.31}, \mathrm{Cl}_{0.025}\right)(\mathrm{OH})_{4}\right] 10$

$\mathrm{K}_{1.09}\left(\mathrm{Mg}, \mathrm{Fe}^{+2}\right)_{5.12}\left(\mathrm{Fe}^{+3}, \mathrm{Al}, \mathrm{Ti}\right)_{0.85}\left[\mathrm{Si}_{5.23} \mathrm{Al}_{2.77} \mathrm{O}_{20}\right]\left[\left(\mathrm{F}_{0.33}, \mathrm{Cl}_{0.032}\right)(\mathrm{OH})_{4}\right] 11$

The average chemical formula is:

$\mathrm{K}_{1.16}\left(\mathrm{Mg}, \mathrm{Fe}^{+2}\right)_{4.96}\left(\mathrm{Fe}^{+3}, \mathrm{Al}, \mathrm{Ti}\right)_{1.00}\left[\mathrm{Si}_{5.23} \mathrm{Al}_{2.77} \mathrm{O}_{20}\right]\left[\left(\mathrm{F}_{0.33}, \mathrm{Cl}_{0.032}\right)(\mathrm{OH})_{4}\right] \quad 12$

The above formula, calculated on the basis of microprobe analysis, identifies that the biotite lies within the annite-siderophyllite composition field. From the available data there is no obvious geographic or stratigraphic variation in biotite composition.

Pyrite. In bulk samples, there are rounded fine- to coarse sand-size clumps of crystalline pyrite. Back-scattered electron microscopy shows that the clumps as well as pyrite that occurs within rounded clay fragments (Fig. 15D) consist of framboids. Similar framboidal pyrite occurs within altered biotite fragments.

Clay minerals. X-ray diffractometry spectra of clay $(<2$ micron $)$ separates from the friable shales (Fig. 16; Table 4), identify the predominance of illite and muscovite, with kaolinite and chlorite as minor constituents, whereas chlorite and illite are both 
Table 4. Relative abundance of minerals defined by XRD analysis in friable mica-shale and compacted shale. Letters in bold designate more abundant minerals. 


\begin{tabular}{|c|c|c|c|c|c|c|}
\hline \multirow{3}{*}{$\frac{\ddot{E}}{\vec{z}}$} & \multirow{3}{*}{ 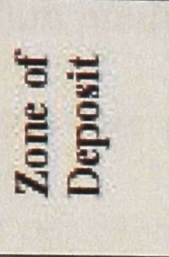 } & \multirow{3}{*}{ Location } & \multirow{3}{*}{$\begin{array}{l}\text { Sample } \\
\text { Number }\end{array}$} & \multicolumn{3}{|c|}{ Mineral Assemblage } \\
\hline & & & & \multicolumn{2}{|c|}{ Random Method } & $\begin{array}{l}\text { Oriented } \\
\text { Method }\end{array}$ \\
\hline & & & & $\begin{array}{c}\text { Clay } \\
\text { minerals }\end{array}$ & $\begin{array}{l}\text { Non-clay } \\
\text { minerals }\end{array}$ & $\begin{array}{c}\text { Clay } \\
\text { minerals }\end{array}$ \\
\hline \multirow{7}{*}{$\frac{\pi}{x}$} & \multirow{6}{*}{ 蕊 } & \multirow[t]{3}{*}{ Russell } & RU3 & $\mathrm{B}, \mathbf{K}, \mathbf{I}, \mathbf{M}$ & $\mathrm{Q}, \mathrm{C}, \mathrm{O}, \mathrm{P}$ & $\mathrm{K}, \mathrm{I}, \mathbf{M}, \mathbf{C h}$ \\
\hline & & & RU5 & $\mathbf{K}, \mathbf{B}, \mathbf{C h}, \mathrm{M}$ & $\mathrm{Q}, \mathrm{C}, \mathrm{O}, \mathrm{P}$ & $\mathrm{K}, \mathrm{B}, \mathrm{Ch}, \mathrm{M}$ \\
\hline & & & RU6 & $\mathrm{K}, \mathrm{I}, \mathrm{Ch}, \mathrm{B}, \mathbf{M}$ & $\mathrm{Q}, \mathrm{C}, \mathrm{P}, \mathrm{O}$ & $\mathrm{K}, \mathrm{I}, \mathrm{Ch}, \mathrm{M}$ \\
\hline & & \multirow{3}{*}{$\begin{array}{l}\text { Billings } \\
\text { Bridge }\end{array}$} & $\mathrm{BB} 10$ & $\mathrm{~K}, \mathbf{M}, \mathbf{B}, \mathrm{Ch}$ & Q, C, O, A, P & $\mathrm{K}, \mathbf{M}, \mathbf{B}, \mathrm{Ch}$ \\
\hline & & & $\mathrm{BB} 12$ & $\mathrm{~K}, \mathbf{M}, \mathrm{B}, \mathrm{Ch}$ & $\mathbf{Q}, \mathbf{C}, \mathrm{O}, \mathrm{A}, \mathbf{P}$ & $\mathrm{K}, \mathrm{M}, \mathrm{B}, \mathrm{Ch}$ \\
\hline & & & $\mathrm{BB} 13$ & $\mathrm{~K}, \mathbf{M}, \mathrm{B}, \mathrm{Ch}$ & $\mathrm{Q}, \mathrm{C}, \mathrm{O}, \mathrm{A}, \mathrm{P}$ & $\mathrm{K}, \mathbf{M}, \mathbf{B}, \mathrm{Ch}$ \\
\hline & Outcrop & Nepean & $\mathrm{NP7}$ & K, I Ch & $\mathrm{Q}, \mathrm{C}, \mathrm{O}, \mathrm{P}$ & $\mathrm{K}, \mathrm{ICh}$ \\
\hline \multirow{13}{*}{$\frac{3}{0}$} & \multirow{7}{*}{ 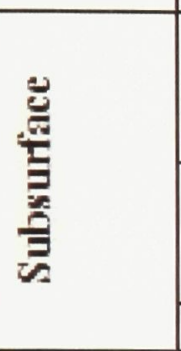 } & \multirow{3}{*}{ Russell } & RU3 & $\bar{K}, \mathbf{I}, \mathbf{M}$ & $\mathbf{C}, \mathbf{Q}$ & $\mathrm{K}, \mathrm{I}, \mathbf{M}$ \\
\hline & & & RU5 & K, I, & $\mathrm{Q}, \mathrm{C}, \mathrm{O}$ & $\mathbf{K}, \mathbf{I}, \mathbf{M}$ \\
\hline & & & RU6 & K, I & Q, C, O & $\bar{K}, \mathbf{I}$ \\
\hline & & \multirow{3}{*}{$\begin{array}{l}\text { Billings } \\
\text { Bridge }\end{array}$} & BB10 & $\mathrm{K}, \mathrm{M}, \mathrm{I}, \mathrm{B}$ & $\mathrm{Q}, \mathrm{C}_{2}$ & $\mathrm{~K}, \mathrm{M}, \mathrm{I}, \mathrm{B}$ \\
\hline & & & BB12 & $\mathrm{K}, \mathbf{I}, \mathbf{B}, \mathbf{M}, \mathrm{Ch}$ & $\mathrm{C}, \mathrm{G}$ & $\mathrm{K}, \mathrm{I}, \mathrm{B}, \mathrm{M}, \mathrm{Ch}$ \\
\hline & & & BB13 & $\mathrm{K}, \mathbf{B}, \mathbf{M}, \mathrm{Ch}$ & Q, C & $\mathrm{K}, \mathrm{B}, \mathbf{M}, \mathrm{Ch}$ \\
\hline & & Lebreton & Leb.15 & $\mathrm{I}, \mathrm{K}, \mathrm{Ch}, \mathrm{M}$ & Q.C & I. $\mathrm{K}, \mathrm{Ch}, \mathrm{MI}$ \\
\hline & \multirow{6}{*}{ 훌 } & \multirow{6}{*}{ Nepean } & NP1 & $\mathrm{K}, \mathrm{I}$, & $\mathrm{Q}, \mathrm{C}, \mathrm{O}$ & $\mathrm{K}, \mathrm{I}$, \\
\hline & & & NP2 & $\mathrm{K}, \mathrm{I}$, & $\mathrm{Q}, \mathrm{C}, \mathrm{O}$ & K, I, \\
\hline & & & NP3 & $\mathrm{K}, \mathrm{I}$, & $\mathrm{Q}, \mathrm{C}, \mathrm{O}$ & $\mathrm{K}, \mathrm{I}$, \\
\hline & & & NP4 & $\mathrm{K}, \mathrm{I}$, & $\mathrm{Q}, \mathrm{C}, \mathrm{O}$ & K, I, \\
\hline & & & NP5 & $\mathrm{K}, \mathrm{I}$, & $\mathrm{Q}, \mathrm{C}, \mathrm{O}$ & $\mathbf{K}, \mathbf{I}$, \\
\hline & & & NP6 & $\mathrm{K}, \mathrm{I}$ & $\mathrm{Q}, \mathrm{C}, \mathrm{O}$ & K, I, \\
\hline \multirow{10}{*}{$\frac{\frac{b}{E}}{\frac{\pi}{\sigma}}$} & \multirow{5}{*}{ 鄫 } & \multirow{4}{*}{ Russell } & RU1 & $\mathrm{I}, \mathrm{K}, \mathrm{Ch}, \mathrm{M}$ & $\mathrm{Q}, \mathrm{C}, \mathrm{D}, \mathrm{P}, \mathrm{Ma}$ & $\mathrm{I}, \mathrm{K}, \mathrm{Ch}, \mathrm{M}$ \\
\hline & & & RU2 & $\mathrm{I}, \mathrm{k}, \mathrm{M}, \mathrm{Ch}$ & $\mathrm{C}, \mathrm{Q}, \mathrm{P}, \mathrm{D}, \mathrm{Mg}$ & $\mathrm{I}, \mathrm{k}, \mathrm{M}, \mathrm{Ch}$ \\
\hline & & & RU7 & $\mathrm{I}, \mathrm{K}, \mathrm{M}, \mathrm{Ch}$ & C, Q, Ma D, P & $\mathrm{I}, \mathrm{K}, \mathrm{M}, \mathrm{Ch}$ \\
\hline & & & RUS & $\mathrm{I}, \mathrm{K}, \mathrm{M}, \mathrm{Ch}$ & C, Q, Ma, D, P & $\mathrm{I}, \mathrm{K}, \mathrm{M}, \mathrm{Ch}$ \\
\hline & & Lebreton & Leb.15 & I. K, Ch & C, Q, P, A & I $\mathrm{K}, \mathrm{Ch}$ \\
\hline & \multirow{5}{*}{ 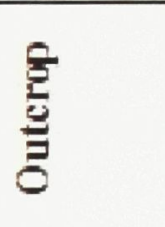 } & \multirow{5}{*}{ Nepean } & NP1 & $\mathbf{K} \mathbf{I}$ & $Q, C, O$ & $\mathbf{K}, \mathbf{I}$, \\
\hline & & & NP2 & K, I, & $\mathrm{Q}, \mathrm{C}, \mathrm{O}$ & $\bar{K}, \mathbf{I}$ \\
\hline & & & NP3 & K, I, & Q, C, O & K, I, \\
\hline & & & NP4 & K. I, & Q, C, O & $\mathrm{K}, \mathrm{I}$ \\
\hline & & & NP5 & K, I & Q, C, O & K. I \\
\hline
\end{tabular}

I=llilite, $\mathrm{K}=$ Kaolinite, $\mathrm{M}=$ mic a (Musc ovite), $\mathrm{Q}=\mathrm{Qu}$ artz, $\mathrm{C}=$ Calcite, $\mathrm{P}=$ Pyrite, $\mathrm{O}=$ Orthoclase $\mathrm{A}=\mathrm{Albite}, \mathrm{G}=$ arpsum, $\mathrm{Ch}=\mathrm{Chlorite}, \mathrm{B}=$ Biotite, $\mathrm{Am}=\mathrm{A}$ mphibole, $\mathrm{Ma}=\mathrm{Maqnefite}$ 
alteration products after biotite (Fig. 15E and F). Within the equivalent shales of the Lebreton core and at Nepean Point, only illite and kaolinite were present, and a small a mount of chlorite, as well (Table 4 and 5).

Chemical formulae for chlorite and illite are summarized below;

\section{Illite:}

1. Russell Core

Upper Ash bed, $\mathrm{N}=3$, the average chemical formula is:

$$
\mathrm{K}_{1.02 \pm 0.04} \mathrm{Al}_{4}\left[\mathrm{Si}_{7.13 \pm 0.17} \mathrm{Al}_{0.86 \pm 0.17+} \mathrm{O}_{20]}(\mathrm{OH})_{4}\right.
$$

Lower Ash bed, $\mathrm{N}=10$, the average chemical formula is:

$$
\mathrm{K}_{1.02 \pm 0.05} \mathrm{Al}_{4}\left[\mathrm{Si}_{7.13 \pm 0.04} \mathrm{Al}_{0.86 \pm 0.04+} \mathrm{O}_{20]}(\mathrm{OH})_{4}\right.
$$

2. Billings Bridge Core:

Upper Ash bed, $\mathrm{N}=3$, the average chemical formula is:

$$
\mathrm{K}_{1.1 \pm 0.00} \mathrm{Al}_{4}\left[\mathrm{Si}_{7.27 \pm 0.02} \mathrm{Al}_{0.73 \pm 0.02+} \mathrm{O}_{20]}(\mathrm{OH})_{4}\right.
$$

Lower Ash bed, $\mathrm{N}=4$, the average chemical formula:

$$
\mathrm{K}_{1.06 \pm 0.04} \mathrm{Al}_{4}\left[\mathrm{Si}_{7.34 \pm 0.1} \mathrm{Al}_{0.66 \pm 0.09+} \mathrm{O}_{20]}(\mathrm{OH})_{4}\right.
$$

\section{Chlorite:}

1. Russell Core

Upper Ash Bed, (RU3), $\mathrm{N}=2$, the average chemical formula:

$\left(\mathrm{Mg}_{3.8 \pm 0.37} \mathrm{Fe}_{2.33 \pm 0.47} \mathrm{Mn}_{0.007 \pm 0.006} \mathrm{Al}_{4.31 \pm 0.42}\right)_{10.45}\left[(\mathrm{Si}, \mathrm{Al})_{8} \mathrm{O}_{20}\right](\mathrm{OH})_{16}$

Lower Ash bed, (RU6 \& RU5), N=6, the average chemical formula

$\left(\mathrm{Mg}_{3.99 \pm 0.21} \mathrm{Fe}_{2.18 \pm 0.00} \mathrm{Mn}_{0.007 \pm 0.001} \mathrm{Al}_{4.37 \pm 0.06}\right)_{10.55}\left[(\mathrm{Si}, \mathrm{Al})_{8} \mathrm{O}_{20}\right](\mathrm{OH})_{16}$

2. Billings Bridge Core:

Upper Ash Bed, (BB10), $\mathrm{N}=2$, the average chemical formula:

$\left(\mathrm{Mg}_{3.23 \pm 0.35} \mathrm{Fe}_{1.91 \pm 0.19} \mathrm{Mn}_{0.004 \pm 0.005} \mathrm{Al}_{4.72 \pm 0.20}\right)_{9.14}\left[(\mathrm{Si}, \mathrm{Al})_{8} \mathrm{O}_{20}\right](\mathrm{OH})_{16}$

Lower Ash Bed, (BB12 \& BB13), N=2, the average chemical formula:

$\left(\mathrm{Mg}_{2.37 \pm 0.79} \mathrm{Fe}_{2.20 \pm 0.02} \mathrm{Mn}_{0.001 \pm 0.001} \mathrm{Al}_{5.11 \pm 0.46}\right.$ 
Table 5. Relative distribution of clay minerals. Biotite, illite, muscovite, kaolinite, and chlorite are abundant in the friable shale, whereas illite and minor kaolinite characterize the clay-mineral composition of the compacted shale.

\begin{tabular}{|c|c|c|c|c|c|c|c|c|c|c|}
\hline \multirow{3}{*}{$\begin{array}{c}\text { Clay } \\
\text { Mineral }\end{array}$} & \multicolumn{3}{|c|}{ ASH } & \multicolumn{4}{|c|}{ CLAY } & \multicolumn{3}{|c|}{ SHALE } \\
\hline & \multicolumn{2}{|c|}{ Subsurface } & \multirow{2}{*}{$\begin{array}{l}\text { Outcrop } \\
\text { Nepean }\end{array}$} & \multicolumn{3}{|c|}{ Subsurface } & \multirow{2}{*}{$\begin{array}{l}\text { Outcrop } \\
\text { Nepean }\end{array}$} & \multicolumn{2}{|c|}{ Subsurface } & \multirow{2}{*}{$\begin{array}{c}\text { Outcrop } \\
\text { Nepean }\end{array}$} \\
\hline & Russell & $\begin{array}{c}\text { Billings } \\
\text { Bridge }\end{array}$ & & Russell & $\begin{array}{c}\text { Billings } \\
\text { Bridge }\end{array}$ & Lebreton & & Russell & Lebreton & \\
\hline Kaolinite & A & $\mathrm{A}$ & A & $\mathrm{A}$ & $\mathrm{C}$ & $\mathrm{C}$ & $\mathrm{A}$ & $\mathrm{C}$ & $\mathrm{C}$ & A \\
\hline Illite & A & $\mathrm{C}$ & A & A & $\mathrm{C}$ & $\mathrm{A}$ & $\mathrm{A}$ & $\mathrm{A}$ & A & A \\
\hline Muscovite & A & A & V.R & A & A & $\mathrm{C}$ & V.R & $\mathrm{C}$ & $\mathrm{R}$ & $\mathrm{C}$ \\
\hline Chlorite & $\mathrm{A}$ & A & $\mathrm{A}$ & & & $\mathrm{C}$ & V.R & $\mathrm{C}$ & $\mathrm{C}$ & V.R \\
\hline Biotite & $\mathrm{A}$ & A & $\mathrm{R}$ & $\mathrm{C}$ & A & V.R & V.R? & V.R & V.R & V.R? \\
\hline
\end{tabular}


Other minerals. Magnetite, apatite, and gypsum, all rare in occurrence, appear restricted to the friable shales and were not found in the compacted shale typical of the Verulam Formation. Magnetite makes up the opaque minerals noted in the bulk sample. In contrast, quartz, feldspar (trace percentages of plagioclase and K-Feldspar) and calcite form major non-clay constituents, but these also occur in the typical Verulam-type compacted shale (Fig. 16; Table 5).

Lithic clasts. Rounded brownish to clear or translucent clasts, many with incorporated angular darker clasts, are lithic fragments. The incorporated fragmental material includes quartz, biotite, as well framboidal pyrite, within a host of clay (illite) (Fig. 15D).

\section{GEOCHEMISTRY}

\section{Bulk rock elemental analysis}

Major oxides: Bulk rock analysis of the friable shales and shale typical of the Verulam Formation are shown in Figs. 17, and Table 6. There is a predominance of $\mathrm{SiO}_{2}$, $\mathrm{Al}_{2} \mathrm{O}_{3}$, and $\mathrm{K}_{2} \mathrm{O}$ and varying abundance of $\mathrm{CaO}$ associated with the friable shales, with a moderate but significant $\left(\mathrm{R}^{2}>0.5\right)$ positive linkage among $\mathrm{SiO}_{2}$ and $\mathrm{Al}_{2} \mathrm{O}_{3}$ and $\mathrm{K}_{2} \mathrm{O}(\mathrm{Fig}$. 17 , plate $1 \mathrm{~A}, \mathrm{C}$; plate $2 \mathrm{D}$ ) that likely reflects the abundance of illite (along with additional muscovite) that forms the abundant mud size sediment. A strong positive correlation $\left(\mathrm{R}^{2}=0.87\right)$ between $\mathrm{MgO}$ and $\mathrm{Al}_{2} \mathrm{O}_{3}$ (Fig. 17, plate $\left.2 \mathrm{~A}\right)$, coupled with a moderate $\left(\mathrm{R}^{2} \sim 0.5\right)$ correlations between $\mathrm{MgO}$ and $\mathrm{SiO}_{2}\left(\right.$ Fig. 17, plate 1D), between $\mathrm{Fe}_{2}$ $\mathrm{O}_{3}$ and $\mathrm{Al}_{2} \mathrm{O}_{3}$ (Fig. 17, plate 2B), and also between $\mathrm{MgO}$ and $\mathrm{Fe}_{2} \mathrm{O}_{3}$ (Fig. 17, plate 3I) likely represents the presence of chlorite (Calvert, 1976) as an alteration product of 
Figure 17. Friable shale and compacted shale oxide geochemistry. For each plate (1-3), the left column contains the friable shale data (Russell, Billings Bridge cores) whereas the right column contains data for equivalent oxide comparisons for the compacted shale (Lebreton, Nepean Point sections). 

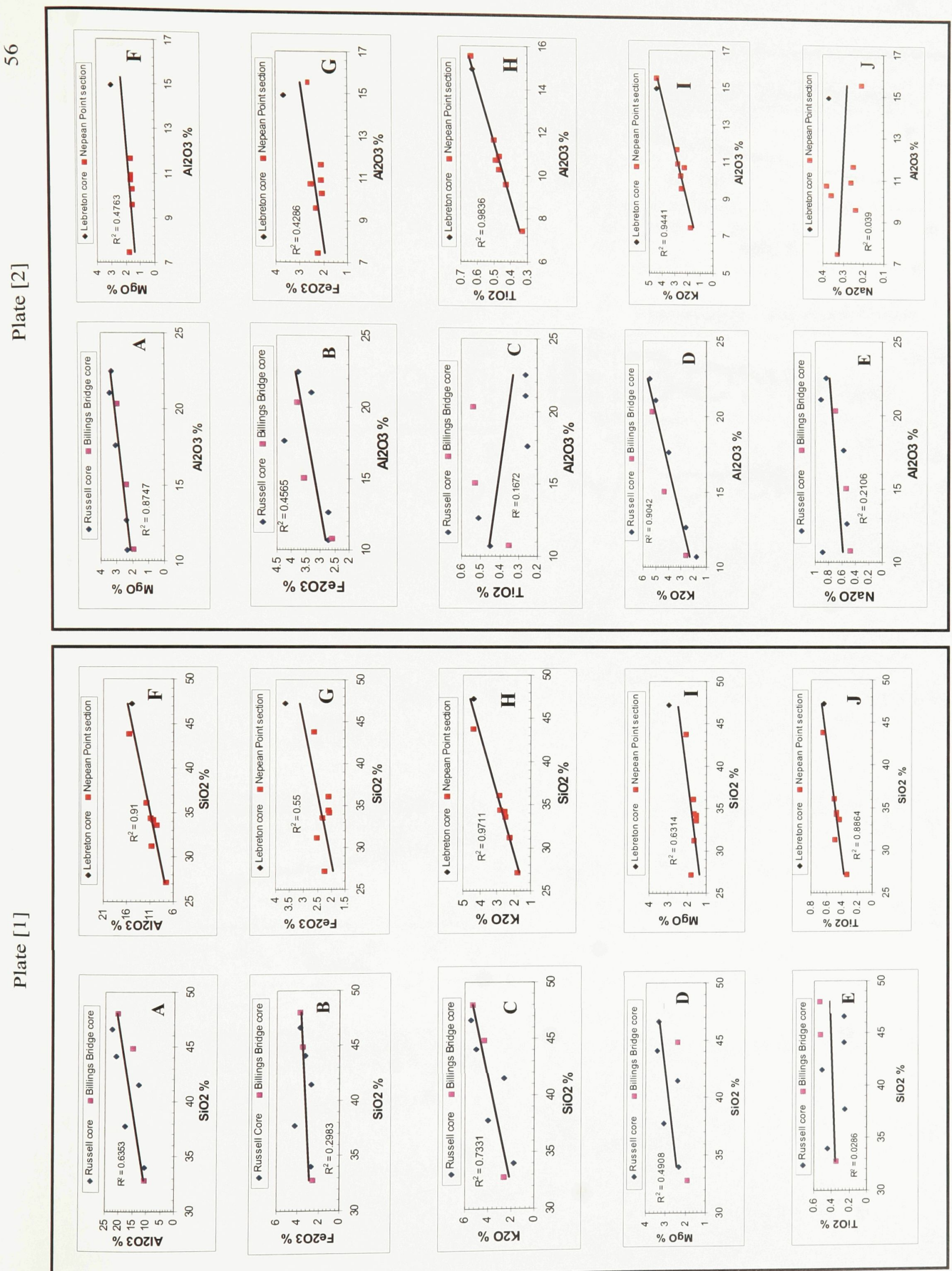

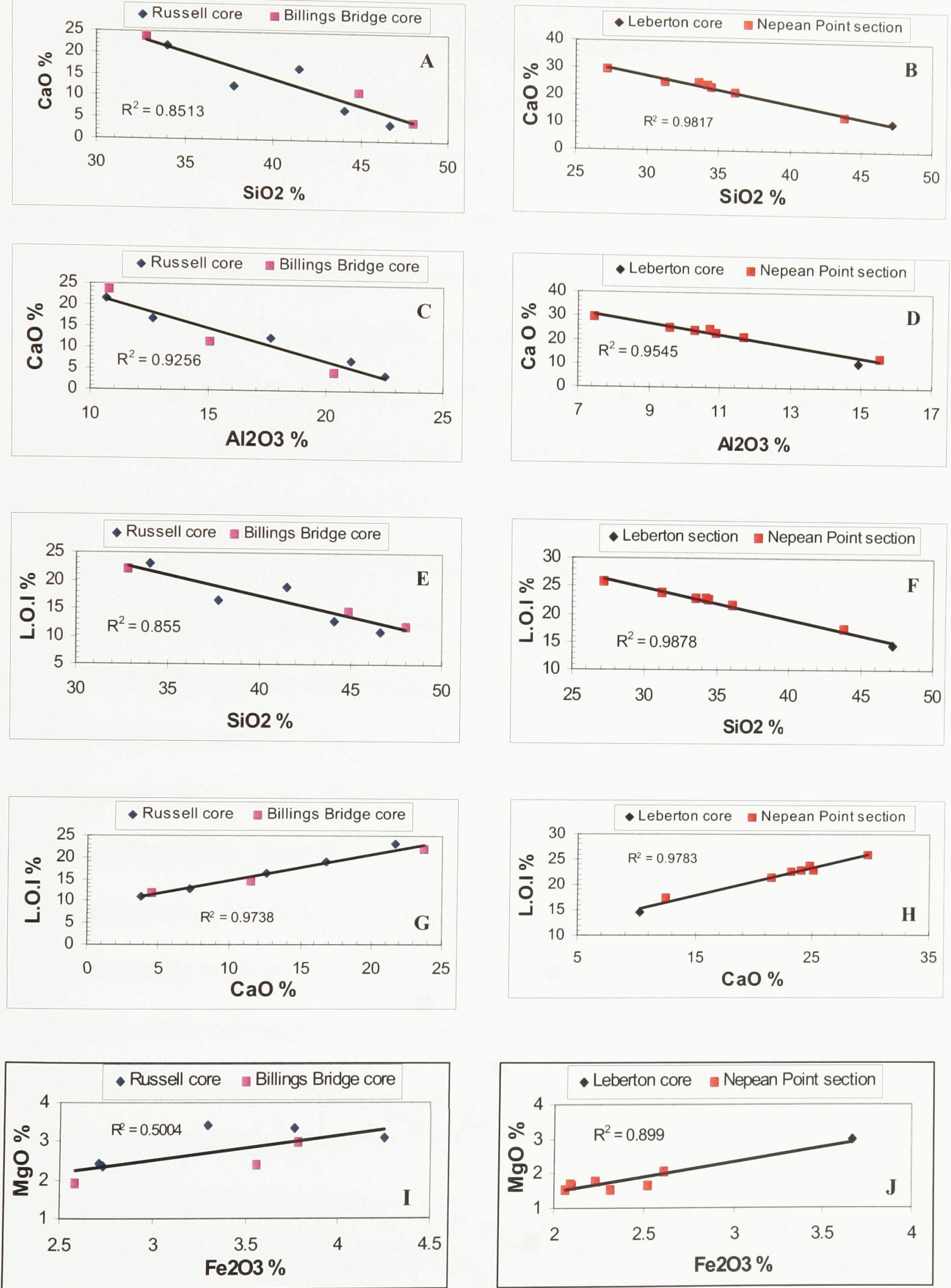
biotite. However, Mg can also substitute within the lattice structure of illite (Calvert, 1976). Given the elevated percentages of clay minerals (kaolinite, muscovite, illite and chlorite) within these rocks, there is a negative correlation between $\mathrm{SiO}_{2}$ and the combination of $\mathrm{CaO}$ and $\mathrm{LOI}$ (=loss on ignition) as well as between $\mathrm{CaO}$ and $\mathrm{Al}_{2} \mathrm{O}_{3}$ (Fig. 17 plate $3 A-G)$. This negative relationship defines loss of volatiles during the XRF analytical process which is independent of silicates. Heating to $1050 \mathrm{C}^{\circ}$ for two hours causes loss of $\mathrm{H}_{2} \mathrm{O}+$ associated with clay mineral structure, sulphur, and drives oxidation of organic matter (releasing $\mathrm{CO}_{2}$ ) above $550^{\circ} \mathrm{C}$. There is an increase in $\mathrm{Si}, \mathrm{Al}$ and $\mathrm{K}$ oxides from the lower to upper friable shale in the Russell core; in the Billings Bridge core the vertical stratigraphic reduction in the abundance of these oxides matches the visible (in core) and measured $(\mathrm{CaO} \%)$ Table 5) increase in carbonate.

Within the compacted shale typical of the Verulam Formation, stronger $\left(\mathrm{R}^{2}>0.8\right)$ correlation coefficients are associated among $\mathrm{SiO}_{2}, \mathrm{Al}_{2} \mathrm{O}_{3}, \mathrm{~K}_{2} \mathrm{O}$, and $\mathrm{TiO}_{2}$, as well as between $\mathrm{MgO}$ and $\mathrm{Fe}_{2} \mathrm{O}_{3}$. The higher correlation coefficient may reflect a greater abundance of illite within the outcrop sections compared to the friable shale. The presence of $\mathrm{TiO}_{2}$ likely identifies the presence of titanium minerals (e.g., Ti-magnetite), but these were not recognized by SEM or XRD. Moderate $\left(\mathrm{R}^{2}>.40\right.$ to .7$)$ positive relationships exist between $\mathrm{SiO}_{2}$ and both $\mathrm{Fe}_{2} \mathrm{O}_{3}$ and $\mathrm{MgO}$, and both these oxides with $\mathrm{Al}_{2} \mathrm{O}_{3}$; again, likely representing the influence of chlorite. The negative association found among $\mathrm{CaO}$, LOI and silicate values is explained as above.

Trace elements and REE. Selected trace elements and rare earth elements (REE), along with ratios are reported in Table 6 and Appendices B, C and D. The elements Th, U, Hf, Zr, Lu, Sm and Y are clearly more abundant within the friable mica- 
shale. Th, U, and $\mathrm{Zr}$ can be linked, at least in part, to the presence of biotite given that zircon inclusions were noted in this mineral (Fig. 15), and pleochroic haloes may be linked to radioactive minerals (Bailey, 1984; Giudotti, 1984). In addition, Th and U are often associated with lattice structure of illite (McLennan et al., 1993). In comparison, the elements $\mathrm{Ba}, \mathrm{Co}, \mathrm{La}, \mathrm{Rb}$, and $\mathrm{V}$ are more concentrated in the compacted shale (Table 6, Appendix D). Ba and $\mathrm{V}$ are often associated with sedimentary deposits and reflect the role of productivity and paleoceanographic conditions (Brumsack 1989; Ballanca et al., 1999). However, $\mathrm{Ba}$ and $\mathrm{Rb}$ can be found to occupy the $\mathrm{Ca}$ and $\mathrm{K}$ position in illite, and the compacted shale will contain more illite on the basis of less porosity and less diverse mineralogy.

The elements $\mathrm{Cr}, \mathrm{V}$, and $\mathrm{Sc}$ are positively correlated with $\mathrm{Al}$ (Appendix E) suggesting that they may be bound within clay structure. In combination with the above, and within the available standard error, the friable mica-shale has lower values of large lithophile elements (LILE: Rb, Ba, Th, Sr. and U) suggesting a different source compared to the compacted shale. This fits with lithological and textural information derived simply from visual examination in core (Fig. 4). The role of trace element analysis in this study is to develop a framework of elements that can be used to characterize the potential source, and this is dealt with in a later section.

REE analysis demonstrates that all samples contain a Eu anomaly, though of different magnitude among samples (Fig. 18). However, the difference in REE abundance between the Billings Bridge and compacted shale suites (Fig. 18) is likely one of greater dilution of REE by quartz or carbonate within the compacted shale. In contrast, the Russell friable shale samples show a range from depletion through enrichment in the 
Table 6. Major oxides, selected ratios and selected elements, bulk friable and compacted shales

\begin{tabular}{|c|c|c|c|c|c|c|c|c|}
\hline \multirow{4}{*}{ Oxides } & \multicolumn{6}{|c|}{ Friable mica-shale } & \multicolumn{2}{|c|}{ Compacted shale } \\
\hline & \multicolumn{3}{|c|}{ Russell Core } & \multicolumn{3}{|c|}{ Billings Bridge Core } & \multirow{3}{*}{$\begin{array}{c}\begin{array}{c}\text { Lebreton } \\
\text { Core }\end{array} \\
\text { Leb. } 16\end{array}$} & \multirow{3}{*}{$\begin{array}{c}\text { Nepean Pt. } \\
\text { section } \\
\begin{array}{c}\mathrm{NP}-7) \\
\mathrm{N}=7\end{array}\end{array}$} \\
\hline & \multirow{2}{*}{$\begin{array}{c}\text { Upper } \\
\text { Bed }\end{array}$} & \multicolumn{2}{|c|}{ Lower Bed } & \multirow{2}{*}{$\begin{array}{c}\begin{array}{c}\text { Upper } \\
\text { Bed }\end{array} \\
\text { BB10 }\end{array}$} & \multicolumn{2}{|c|}{ Lower Bed } & & \\
\hline & & (RU5) & (RU6) & & $\mathrm{BB} 12$ & BB13 & & \\
\hline $\mathrm{SiO}_{2}$ & 37.76 & 44.1 & 46.63 & 48.02 & 44.86 & 32.79 & 47.22 & $33.66(3.44)$ \\
\hline $\mathrm{Al}_{2} \mathrm{O}_{3}$ & 17.62 & 21.06 & 22.5 & 20.34 & 15.06 & 10.78 & 14.92 & $11.18(0.66)$ \\
\hline $\mathrm{Fe}_{2} \mathrm{O}_{3}$ & 4.25 & 3.29 & 3.76 & 3.78 & 3.56 & 2.58 & 3.67 & $2.31(0.30)$ \\
\hline MnO & 0.02 & 0.015 & 0.01 & 0.02 & 0.023 & 0.029 & 0.03 & $0.03(0.0)$ \\
\hline MgO & 3.08 & 3.42 & 3.34 & 2.97 & 2.4 & 1.9 & 2.97 & $1.66(0.03)$ \\
\hline $\mathrm{CaO}$ & 12.53 & 7.18 & 3.81 & 4.51 & 11.45 & 23.67 & 10.26 & $23.12(2.35)$ \\
\hline $\mathrm{Na}_{2} \mathrm{O}$ & 0.57 & 0.91 & 0.84 & 0.70 & 0.54 & 0.48 & 0.37 & $0.32(0.09)$ \\
\hline $\mathrm{K}_{2} \mathrm{O}$ & 3.98 & 5 & 5.49 & 5.3 & 4.34 & 2.61 & 4.41 & $2.54(0.41)$ \\
\hline $\mathrm{TiO}_{2}$ & 0.25 & 0.26 & 0.26 & 0.54 & 0.53 & 0.35 & 0.63 & $0.50(0.0)$ \\
\hline $\mathrm{P}_{2} \mathrm{O}_{5}$ & 0.2 & 0.17 & 0.15 & 0.13 & 0.15 & 0.16 & 0.11 & $0.18(0.06)$ \\
\hline $\mathrm{Cr}_{2} \mathrm{O}_{3}$ & $<0.01$ & 0.01 & $<0.01$ & $<0.01$ & $<0.01$ & $<0.01$ & 0.01 & $<0.01$ \\
\hline LOI & 16.41 & 12.76 & 10.96 & 11.94 & 14.63 & 22.07 & 14.41 & $23.8(1.62)$ \\
\hline TOTAL & 96.68 & 98.17 & 97.75 & 98.26 & 97.54 & 97.41 & 99.01 & 99.31 \\
\hline Ba & 94.9 & 10 & 46.2 & 73.3 & 110.2 & 79.1 & 305.6 & 291.7 \\
\hline Co & 3.4 & 3 & 4.3 & 5.1 & 6.2 & 4.7 & 10 & 7.2 \\
\hline $\mathrm{Cr}$ & $<24$ & $<24$ & $<24$ & $<24$ & 37 & 24 & 87 & 73 \\
\hline Eu & 0.38 & 0.11 & 0.403 & 0.94 & 0.822 & 0.866 & 0.45 & 0.45 \\
\hline Hf & 4.28 & 8.14 & 7.14 & 10.4 & 7.63 & 5.85 & 3.77 & 2.54 \\
\hline La & 13.84 & 1.39 & 9.04 & 18.59 & 26.34 & 25.48 & 26.5 & 19.28 \\
\hline Lu & 0.36 & 0.191 & 0.28 & 0.33 & 0.316 & 0.328 & 0.17 & 0.15 \\
\hline Nb & 4.78 & 8 & 9.25 & 19.61 & 16.58 & 11.5 & 11.43 & 9.17 \\
\hline $\mathbf{R b}$ & 79.6 & 18.8 & 42.6 & 77.2 & 159.7 & 116.1 & 194.2 & 143.4 \\
\hline Sc & 7.5 & 4.5 & 9.6 & 15.8 & 10.3 & 10.3 & 11.4 & 11 \\
\hline Sm & 4.29 & 1.09 & 4.07 & 4.42 & 4.28 & 4.72 & 2.29 & 2.31 \\
\hline Sr & 564 & 90 & 203 & 303 & 417 & 455 & 341 & 390 \\
\hline Th & 20.41 & 7.37 & 31.34 & 19.97 & 13.22 & 11.89 & 11.79 & 8.91 \\
\hline $\mathbf{U}$ & 4.45 & 10.33 & 9.89 & 3.11 & 2.23 & 1.85 & 1.7 & 1.83 \\
\hline $\mathbf{V}$ & 13 & 16 & 19 & 35 & 38 & 29 & 63 & 55 \\
\hline $\mathbf{Y}$ & 21.77 & 9.23 & 19.28 & 19.92 & 17.32 & 18.66 & 8.32 & 8.9 \\
\hline $\mathbf{Z n}$ & 28 & 22 & 36 & 16 & 25 & 21 & 27 & 18 \\
\hline $\mathrm{Zr}$ & 136 & 221 & 199 & 362 & 294 & 214 & 141 & 93 \\
\hline $\mathrm{K} 2 \mathrm{O} / \mathrm{Na}_{2} \mathrm{O}$ & 6.98 & 5.49 & 6.53 & 7.57 & 8.04 & 5.44 & 11.92 & 7.94 \\
\hline $\mathrm{CaO} / \mathrm{Na}_{2} \mathrm{O}$ & 21.98 & 7.89 & 4.54 & 6.44 & 21.37 & 49.31 & 27.73 & 72.25 \\
\hline $\mathrm{SiO}_{2} / \mathrm{Al}_{2} \mathrm{O}_{3}$ & 2.14 & 2.09 & 2.07 & 2.36 & 2.98 & 3.04 & 3.16 & 3.01 \\
\hline
\end{tabular}


Figure 18. Pattern of REE-Chondrite for bulk ash samples of friable and compacted shales showing the enrichment in LREE over HREE. RU5 sample revealed more depleted in LREE and has a longer negative Eu anomaly, and that suggest the depletion in trace element indicate depletion in monazite mineral. 


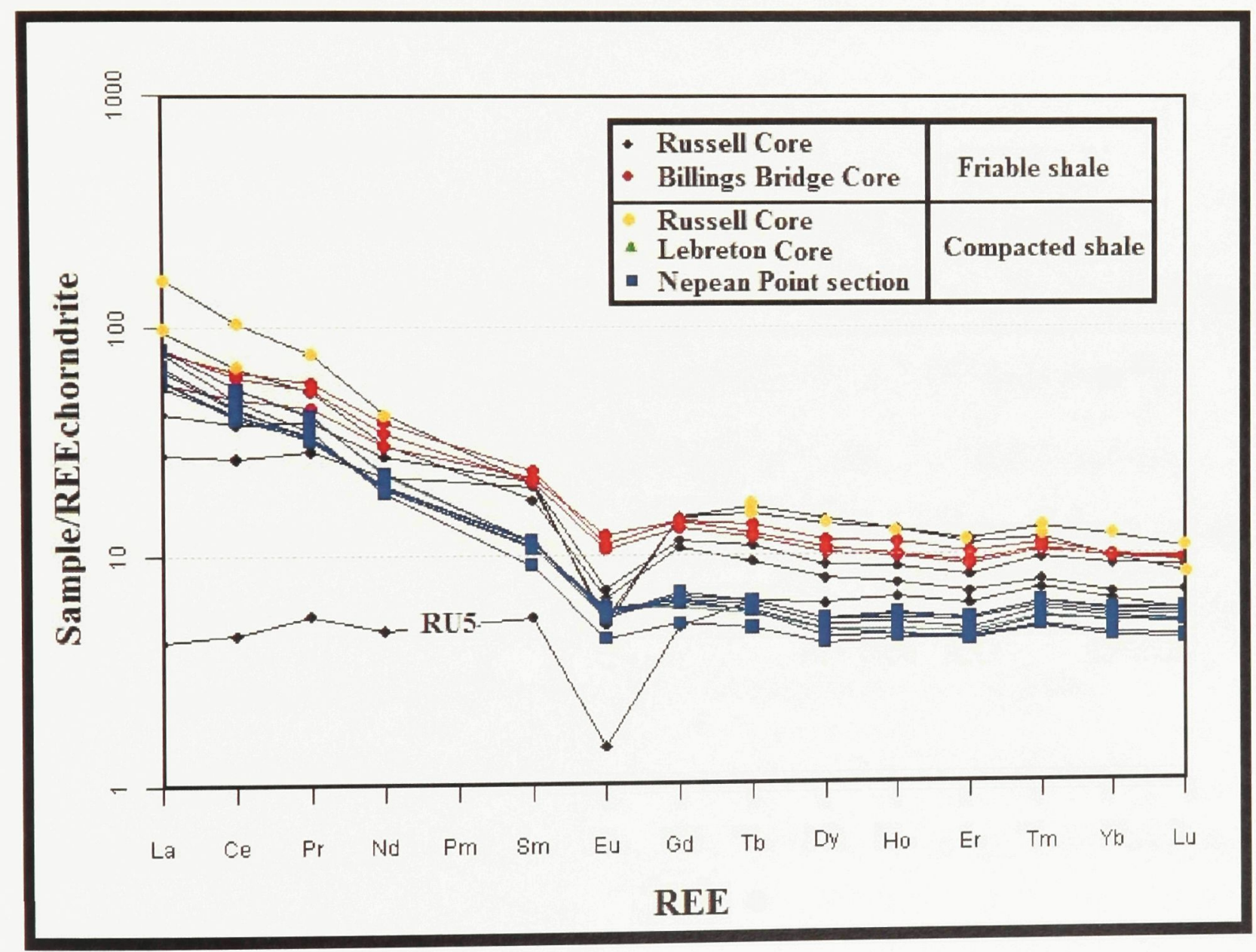


LREE range, with sample RU5 possessing a slightly negative LREE gradient. Flat or negative LREE gradients can be associated with mafic (e.g., basaltic) magmas, but also often associated with felsic magmatic liquid in which monazite has precipitated (Rollinson, 1995), reducing the LREE suite. Interestingly, however, both seawater and meteoric water show a similar LREE decline, yet alteration of REE values in sedimentary rocks through diagenesis is viewed as unlikely given that large water/rock ratios that are required.

\section{Interbedded Carbonate}

Stable (C,O) Isotope Analysis. All but one sample are of bulk rock, the exception being of diagenetic calcite from within the friable shale. One sample (RU-14a) is of brachiopod calcite, with $\delta^{13} \mathrm{C}$ and $\delta^{18} \mathrm{O}$ values of -0.14 and $-6.8 \%$ respectively. Stratigraphic variation relative to the Billings Bridge core (Fig. 19, Table 7) shows a marked positive shift in $\delta^{13} \mathrm{C}^{\prime}$ values, relative to $\mathrm{PDB}$, from $\sim-0.2 \%$ to $\sim<0.1 \%$, across the base of the lower friable shale. A matching $\left(\mathrm{R}^{2}=0.721\right)$ positive shift, but from -7.4 to $-6.5 \%$, is associated with $\delta^{18} \mathrm{O}$ over the whole core interval that was sampled.

Mean stable $(\mathrm{C}, \mathrm{O})$ isotope values for Late Ordovician (Caradoc/Mohawkian) brachiopods (shown along with maximum and minimum values; Shields et al., 2003) are compared to the Verulam sample range:

\begin{tabular}{|c|c|c|c|c|}
\hline $\begin{array}{c}\text { Global Signature } \\
\text { brachiopod }\end{array}$ & $\% 0)$ & \multicolumn{3}{|c|}{$\begin{array}{c}\text { Billings Bridge ash-bed interval (\%o) } \\
\text { skeletal }+ \text { cement }\end{array}$} \\
\hline mean $=0.1$ & (1.6 to -2.1$)$ & mean & -0.1 & -0.2 to 0.1 \\
\hline mean $=-4.8$ & $(-3.1$ to -6.8$)$ & mean & -6.8 & -7.4 to -6.7 \\
\hline
\end{tabular}


Figure $19 . \delta{ }^{18} \mathrm{O}$ and $\delta{ }^{13} \mathrm{C}$ values of bulk sample consisting of mixed brachiopods and interstitial cement. 

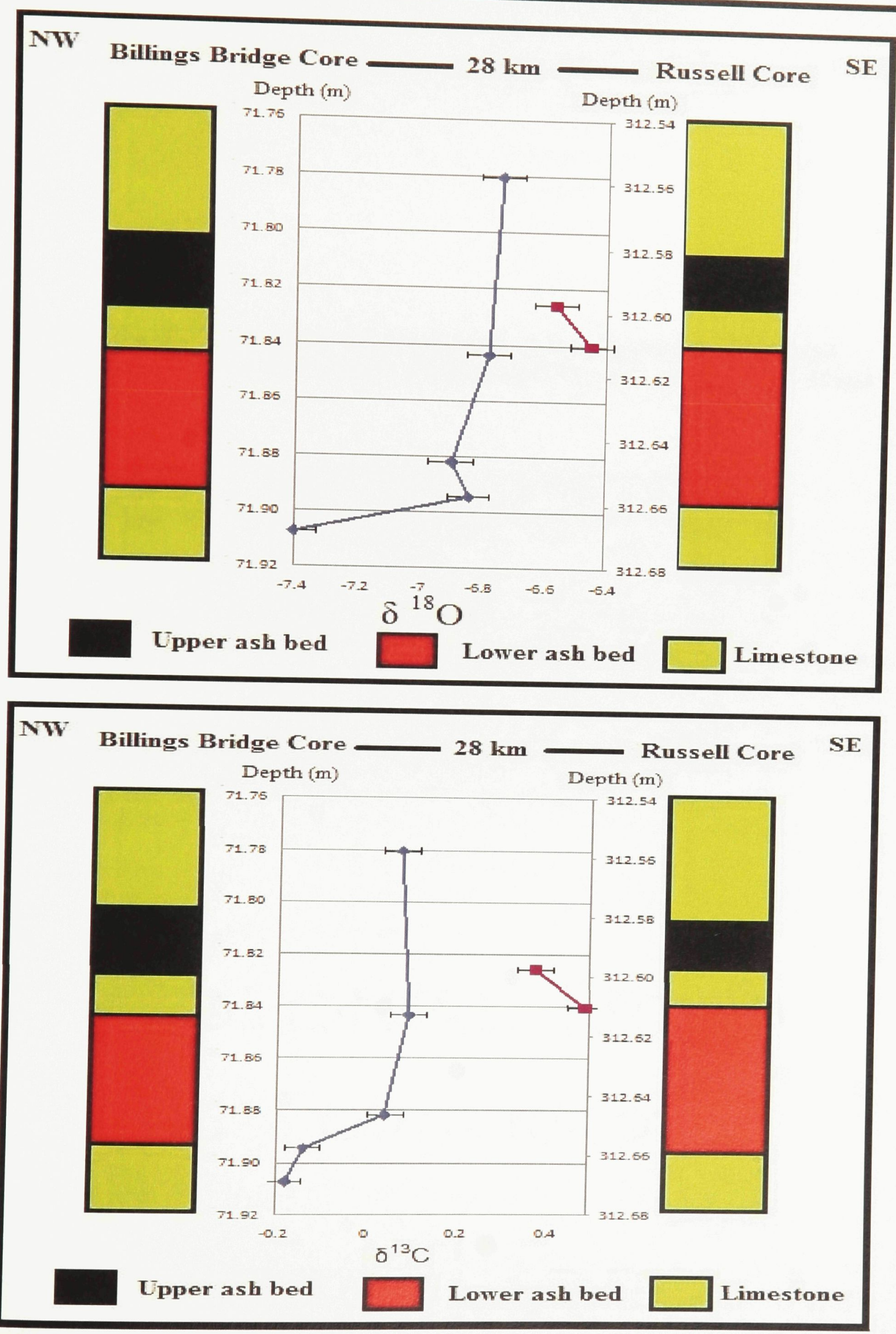
Table 7. $\delta{ }^{18} \mathrm{O}$ and $\delta{ }^{13} \mathrm{C}$ signatures of the carbonate rocks in Russel Core (RU4), and Billings Bridge core (BB9, BB11, BB13, BB14 and BB14a). Std error is $0.1 \% \delta^{18} \mathrm{O}$ and $\delta{ }^{13} \mathrm{C}$.

\begin{tabular}{|c|c|c|c|c|c|}
\hline Sample & Depth & Depth ft & Depth $\mathrm{m}$ & $\delta{ }^{13} \mathrm{C} \%$ & $\delta{ }^{18} \mathrm{O} \% 0$ \\
\hline $\begin{array}{c}\text { For } \\
\text { RU4 }\end{array}$ & $1025^{\prime} 7^{\prime \prime}$ & 1025.58 & 312.60 & 0.37 & -6.56 \\
\hline RU4a & $1025^{\prime} 7.5^{\prime}$ & 1025.63 & 312.61 & 0.48 & -6.45 \\
\hline BB9 & $235^{\prime} 6^{\prime \prime}$ & 235.50 & 71.78 & 0.07 & -6.74 \\
\hline BB11 & $235^{`} 8.5^{\prime \prime}$ & 235.71 & 71.84 & 0.09 & -6.78 \\
\hline BB13 & $235^{`} 10^{\prime \prime}$ & 235.83 & 71.88 & 0.04 & -6.9 \\
\hline BB14 & $235^{`} 10.5^{\prime \prime}$ & 235.88 & 71.89 & -0.14 & -6.84 \\
\hline BB14a & $235^{`} 11^{\prime \prime}$ & 235.92 & 71.91 & -0.18 & -7.4 \\
\hline
\end{tabular}


It appears that there has been diagenetic influence causing a negative shift in the oxygen isotope signature from the expected marine field, with a lesser shift in carbon or carbonate isotope signature.

Trace elements. Sr and Mn concentrations of bulk (skeletal and calcite cement) carbonate (Fig. 20) show differences in the fields defined for the Russell and Billings Bridge cores, with the latter having a higher $\mathrm{Mn} / \mathrm{Sr}$ ratio associated with lower $\delta^{13} \mathrm{C}$. This combined negative shift also reflects a greater degree of alteration in the presence of burial fluids (Brand and Veizer, 1981; Kaufmann and Knoll, 1995).

\section{Ar-Ar DATING: BIOTITE}

Three biotite grains selected for Ar-Ar analysis from the lower and upper friable shales in the Russell core yielded saddle-shaped release spectra (Fig.21). This type of result does not allow an age interpretation. The obvious alteration of biotite to illite and chlorite as illustrated by electron microscopy (Fig. 15) explains the analytical failure. As mentioned, the need to rapidly provide samples to the Geological Survey of Canada prior to sufficient examination of the material forms the basis of this failing. 
Figure 20: Bulk-rock elemental and stable isotope data, Verulam limestone. (A) $\delta 18$ Ocarbonate versus $\mathrm{Mn} / \mathrm{Sr}$. (B) $\delta 13 \mathrm{Ccarbonate}$ versus $\mathrm{Mn} / \mathrm{Sr}$. The Billings Bridge samples define a greater negative shift in both isotopes relative to elevated $\mathrm{Mn} / \mathrm{Sr}$ ratio, suggesting greater diagenetic influence. 


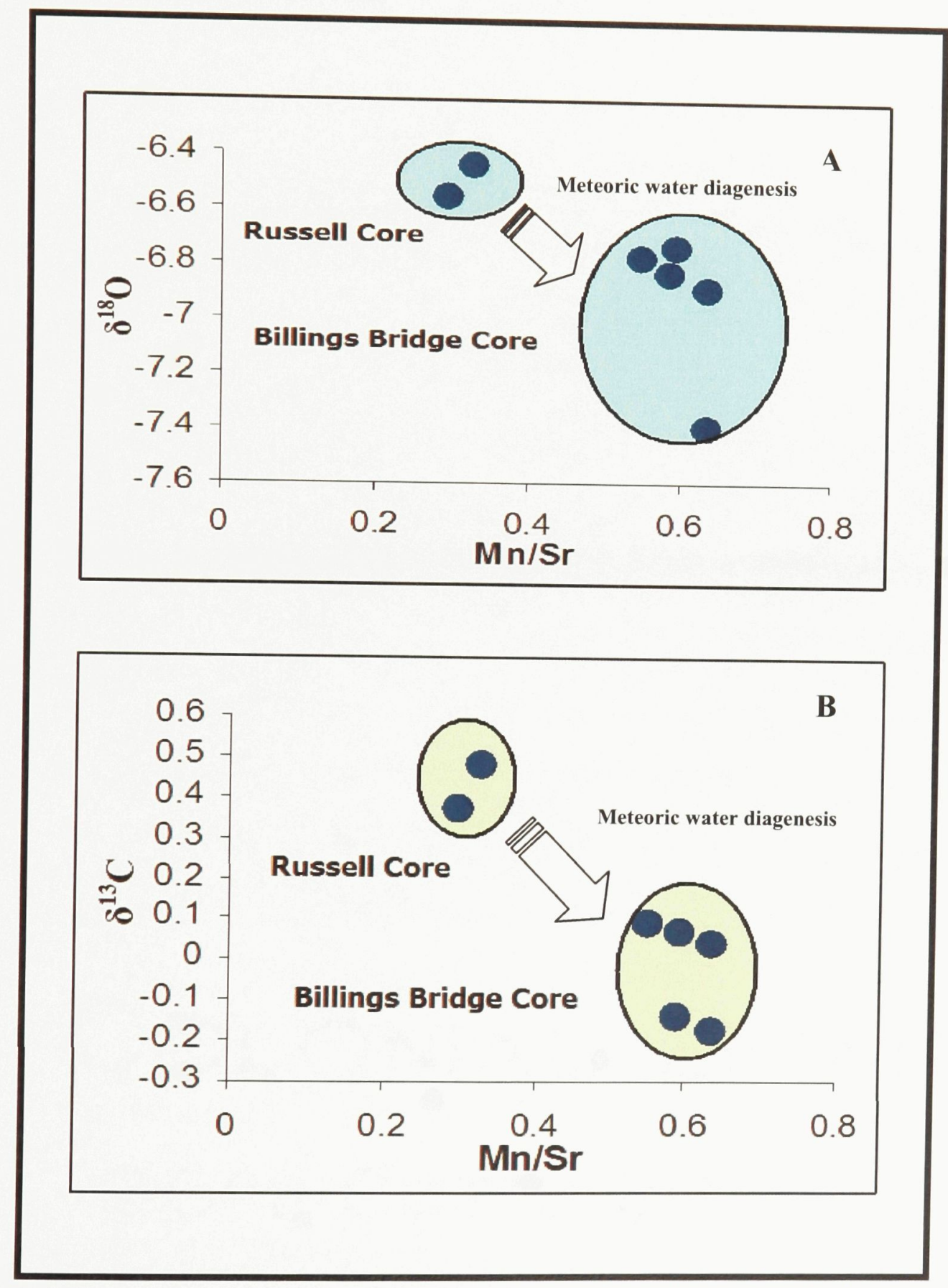


Figure 21. Failure age-dating using Ar-Ar method of biotite in friable shales. 


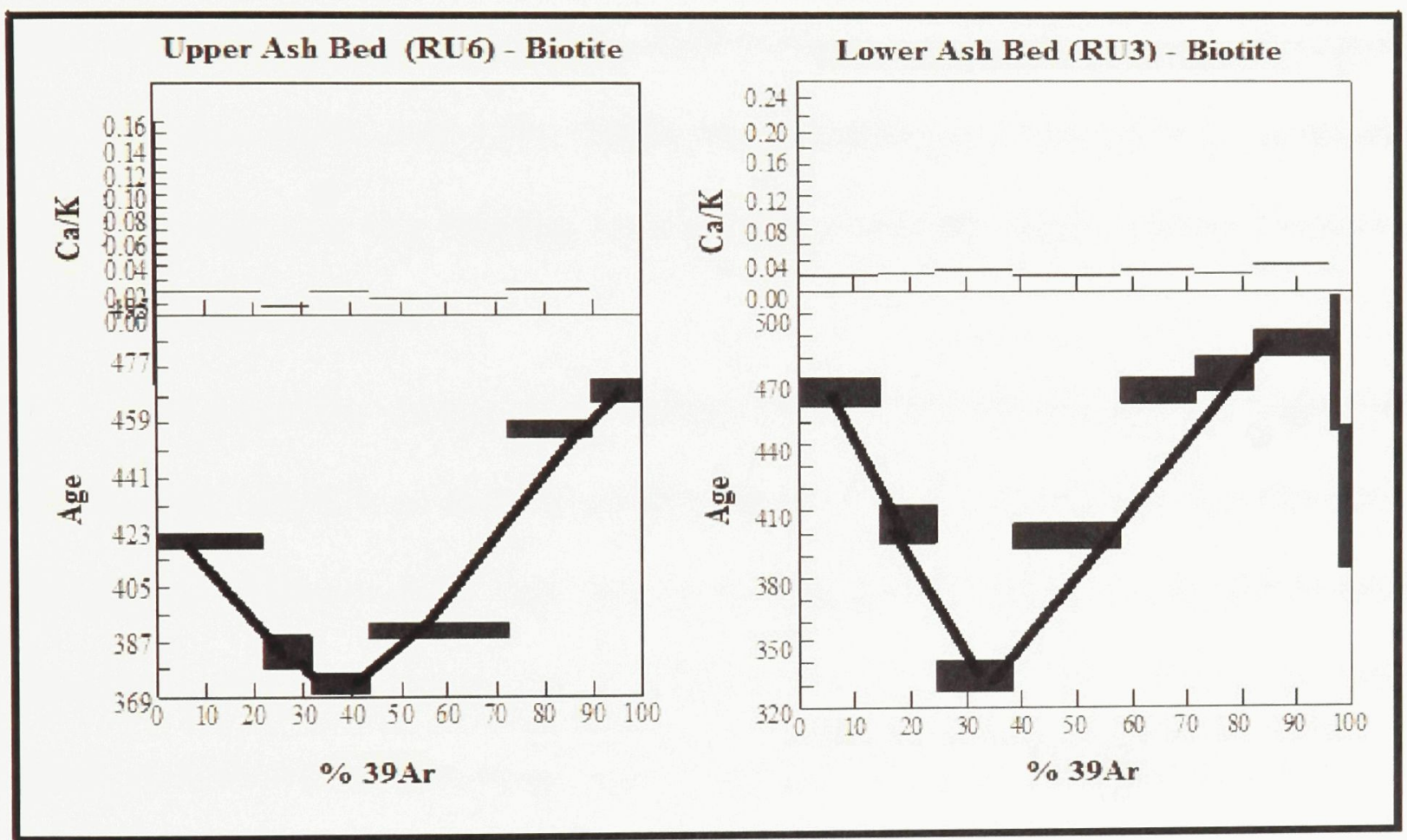




\section{ORIGIN OF THE VERULAM FRIABLE SHALE}

Visual, lithic, and geochemical attributes all demonstrate that the friable shale is of a different origin than the typical compacted shale of the Verulam Formation. Within the Upper Ordovician foreland basin along eastern North America, the additional depositional component of the vast carbonate platforms, which incorporate shale and minor sandstones as a function of sea level variation, are altered volcanic ash deposits, now bentonites (Huff et al. 1992; 2008). These deposits are typically thin (centimetrescale) and often only clay-bearing; some are illite-bentonites, others contain mixtures of illite and montmorillonite.

The following stratigraphic, lithological, and geochemical proxies demonstrate that the friable shale is an altered volcanic ash deposit. There is then an interpretation of the magmatic source along with how these deposits fit the evolving framework of volcanism along the eastern margin of Laurentia, and, then, the tectonostratigraphic significance of such volcanism on the development of the Laurentian foreland basin.

\section{THE VERULAM FRIABLE SHALES AS ALTERED VOLCANIC ASH Stratigraphic and lithological evidence}

Regional subsurface geophysical correlation of the Russell friable shale interval (Fig. 9) demonstrates a potential widespread occurrence of this unit extending from the Michigan Basin to southern Quebec, a distance of $\sim 1000 \mathrm{~km}$. Over this distance the geophysically defined interval is very thin, (Fig. 9), and in the Russell core $\sim 6 \mathrm{~cm}$ in thickness.

Studies of Quaternary ash deposits identify an overall decrease in grain size from source (Riley et al., 2003). The grain size reduction of biotite and lithic grains moving 
from the Russell to Billings Bridge sites, therefore, suggests waning transport along a direction $\left(290^{\circ}\right)$ that would indicate a potential source to the ESE. The origin of the lithic grains remains uncertain; they may be altered pumice fragments, given the prominent clay matrix (Fig. 15D). Pumice fragments would be very metastable and readily alter to clay (Sharma et al., 2005).

The double layer of friable shale illustrates that there were likely two eruptive events, the first being longer or containing more ash. The visually defined presence of increased skeletal carbonate within the upper bed at the Billings Bridge site compared to the equivalent bed in the Russell core might illustrate lesser NW transport of volcanic material and-or a decrease in settling rate of volcanic material. The absence of an altered ash bed in the Lebreton core and at Nepean Point is unexpected. It may have been eroded, with trace biotite defining only an erosional remnant; or, it was strongly diluted by background shale, although this requires a very elevated lateral gradient (over $3 \mathrm{~km}$ ) in dilution compared to the Russell to Billings Bridge $(24 \mathrm{~km})$ transect.

Alternatively, apart from the rare coarser-grained components, the ash was not deposited due to wave action or currents. Models of ash-laden seawater (Carey, 1997) demonstrate that it does not settle passively but accumulates in the air-water boundary layer until a density limit is exceeded creating vertical gravity currents (Fig. 4 in Carey, 1997). The absence of discreet altered ash beds in the Lebreton and Nepean Point sections may indicate, therefore, not the distal limit of the ash cloud, but the distal limit at which fair-weather wave or current action became sufficient (in comparison to the NWdecrease in ash transport) to dissipate ash and not exceed the critical limit of density plume formation. 
Geochemical proxies. There are a series of proxies used to help identify the potential magmatic source of altered volcanic ash (e.g., Huff et al., 1992; Haynes et al., 1995; Batchelor, 2003). These proxies are elemental plots associated with igneous endmember compositions that assume that elements carried by the original depositional components are retained, or mostly so, through diagenesis. Thus, these proxies are, at best, guides to general magmatic sources.

In general, these proxies demonstrate that the Verulam altered ash deposit reflects an andesitic source, one that is compatible with a source from the evolving volcanic arc adjacent to the Laurentian plate margin. Evidence is as follows:

a) $\mathrm{Al}_{2} \mathrm{O}_{3}-\mathrm{FeO}$ (Abdel-Rahman, 1994): A magmatic discrimination diagram (Fig. 22A) separates alkaline, peraluminous, and calc-alkaline groups of igneous rocks, and shows the bulk sample geochemistry of the Verulam deposits bracketing the division between peraluminous and calc-alkaline rocks.

b) $1 / 3 \mathrm{Hf}-\mathrm{Th}-\mathrm{Ta} / 16$ (Wood, 1980): This relationship (Fig. 22B) discriminates tectonic setting of volcanic rocks, and shows nearly all the data points from the bulk sample geochemistry falling well within the continental-activeboundary $(\mathrm{CAB})$ field.

c) $\mathrm{Zr} / \mathrm{TiO} 2$ vs Nb/Y (Winchester and Floyd, 1997): Bulk rock analysis reveals breadth of data occurring within the trachyandesite - rhyodacite/dacite fields (Fig. 22C). 
d) Nb vs Zr (Leat et al., 1986): These authors propose a division of volcanic rocks according to magmatic alkalinity (subalkaline, peralkaline). The Verulam data fall within their High-K, Subalkaline field.

e) $\mathrm{Rb}$ vs $(\mathrm{Ta}+\mathrm{Yb})$ (Pearce et al., 1984): This discrimination plot (Fig. 22D) separates granite types according to tectonic setting. The bulk rock data fall within the volcanic arc granite (VAG).

\section{Comparison with peri-lapetan Late Ordovician - Early Silurian volcanism}

Altered volcanic ash beds are common throughout the Upper Ordovician foreland carbonate platform succession and extending into the Early Silurian, all related to arc volcanism during the destructive phase of the Iapetan Ocean basin. Although many bentonite layers have been reported, only a few have been studied in detail (Haynes et al., 1995; Huff, 1996; Min et al., 2001; Batchelor, 2003; Sharma et al., 2005; this study). Of these, three events dominate: Deicke and Millbrig (USA, Canada), and Kinnekulle (Sweden). These three events, older than Verulam deposition, bracket the interval equivalent to the Chaumont Formation (Fig. 4), with the latter two events possibly being contemporaneous (Huff et al., 1992; Bergstrom et al., 2004) forming one of the largest volcanic eruptive events in geologic history (Fig. 23). Within the fallout area in North America, there is a NW-directed loss of metastable clay mineralogy (Fig. 23). 
Figure 22. Tectonomagmatic discrimination diagrams of A) biotite and $\mathrm{B}-\mathrm{D}$ ) bulk rock samples. A) $\mathrm{Al}_{2} \mathrm{O}_{3}-\mathrm{FeO}$ magmatic discrimination diagram for biotite composition based on Abdel-Rahman (1994), wherein the Verulam biotite brackets the division between peraluminous and calc-alkaline rocks. B) ${ }_{1 / 3} \mathrm{Hf}-\mathrm{Th}-\mathrm{Ta} / 16$ of Wood (1980) showing that the bulk sample fall well within the continental-active-boundary (CAB) field. C) $\mathrm{Zr} / \mathrm{TiO}_{2} \mathrm{vs} . \mathrm{Nb} / \mathrm{Y}$ of Winchester and Floyd (1997), indicating that the bulk rock samples occur within the trachyandesite - rhyodacite/dacite fields. D) Rb vs. ( $\mathrm{Ta}+\mathrm{Yb}$ ) of Pearce et al., (1984), showing that the bulk rock data fall within the volcanic arc granite (VAG). 


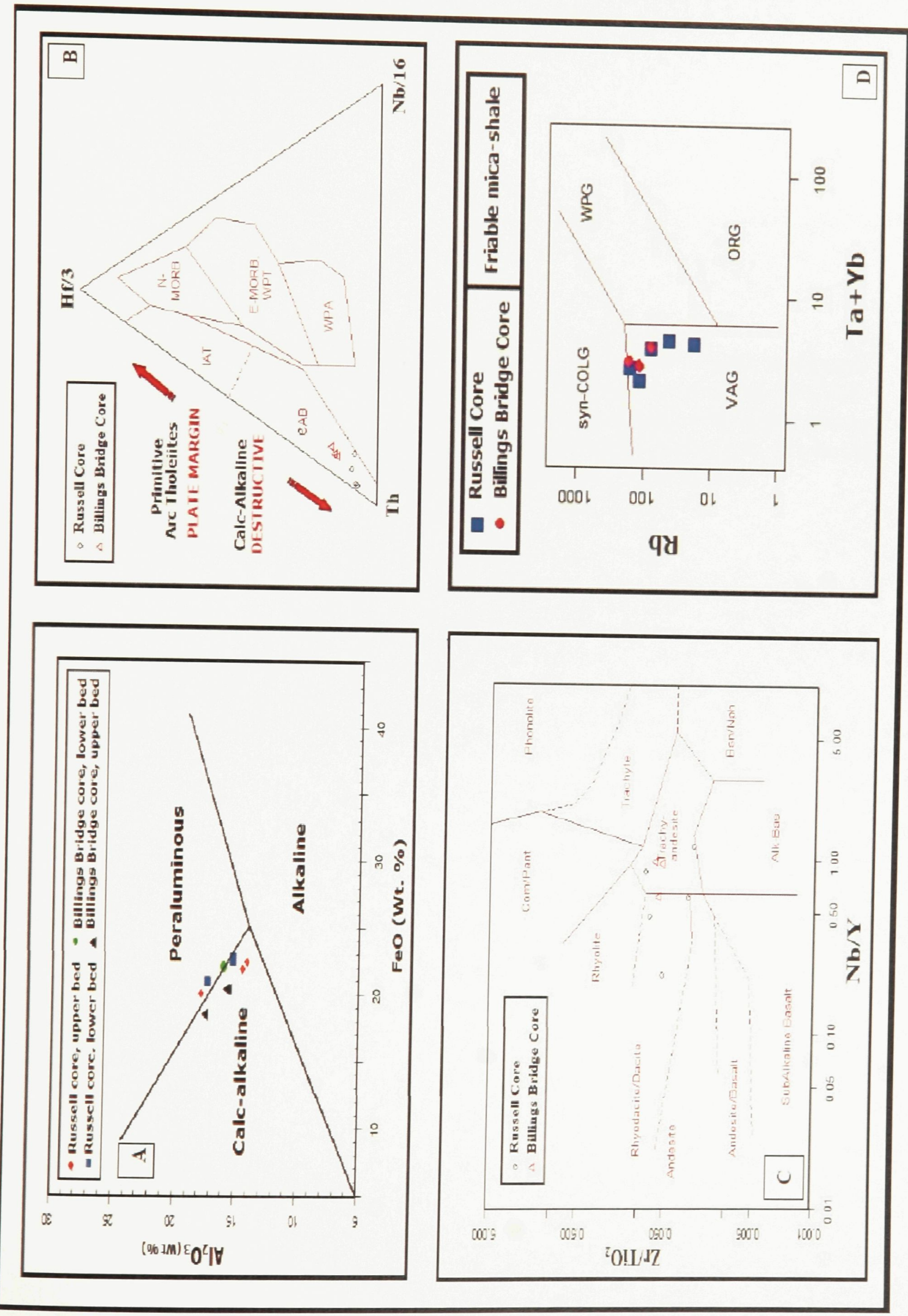


Figure 23 Postulated areal distribution of the Millbrig + Kinnekulle events in the periIapetan region. The green lines show the thickness in $\mathrm{cm}$ based on different locations (black dots) (Huff et al. 1996), and the red lines illustrate changing mineralogy moving from proximal to distal positions: Group 1 (GP.1 on the map), smectite plus S/I mixed layer (with $\mathrm{S}>\mathrm{I}$ ); Group 2 (Gp.2 on the map), smectite $+\mathrm{S} / \mathrm{I} \%$ (with I $>\mathrm{S}$ ). 


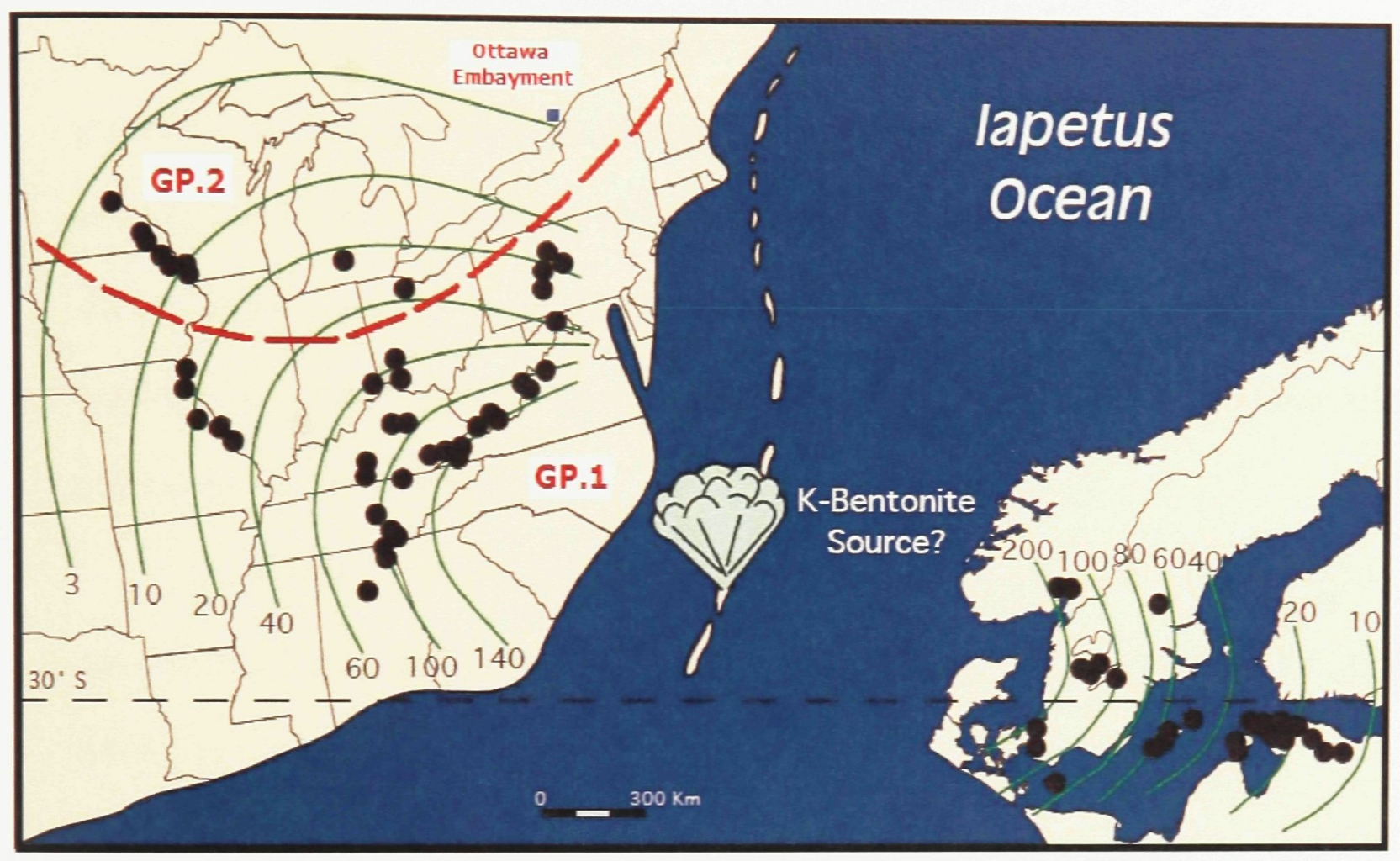


Figure 24 shows a series of bivariate geochemical plots showing the distribution of the Millbrig, Deicke, and Kinnekulle events (Huff, 2008) in comparison to the Verulam dataset (this study). Despite the obvious age difference between the first three and the Verulam altered ash there are strong overlapping fields illustrating likely similar geochemical source and-or post-depositional alteration as influenced by primary geochemistry. The datasets show that the Verulam field lies within that for the combined Millbrig and Kinnekulle whereas the Deicke field is mostly separate: this latter event is shifted farther into the calk-alkaline field and has a higher $\mathrm{Mg} \# \mathrm{Mg} \#$ (defined as: $\mathrm{Mg} / \mathrm{Mg}+\mathrm{Fe}$ ) or $\mathrm{MgO}$ content. The more elevated $\mathrm{Mg} \#$ refers to a less fractionated magma (Haynes et al. 1995; Huff, 2008).

Incorporating all available data related to altered volcanic ash deposits for the peri-Iapetan Upper Ordovician and a Lower Silurian succession (Fig. 25; Table 8 and 9) reveals the following:

a) biotite is the prominent related mica within volcanic ash deposits hosted by the carbonate foreland successions, whereas the more Mg-bearing phlogopite dominates the foreland shale basin and Lower Silurian platform successions (Fig. 25A).

b) $\mathrm{FeO}$ and $\mathrm{MgO}$ percentages (Fig. 25B) show two groups (Group I: Deicke, separated from younger (Group II) platform-hosted volcanic ash deposits, both different from samples (Group III) derived from the Ottawa Embayment foreland shale basin (Sharma et al., 2005) and Lower Silurian sections in western Europe and Scotland (Batchelor, 2003). 
Figure 24. Bivariate plots of oxides showing distribution of the Millbrig, Deicke, and Kinnekulle events compared with the Verulam dataset. Each of the fields for the older events contain $95 \%$ of all biotite samples reported by Huff (2008). The data suggest that the Deicke is of a slightly different magmatic source type. 

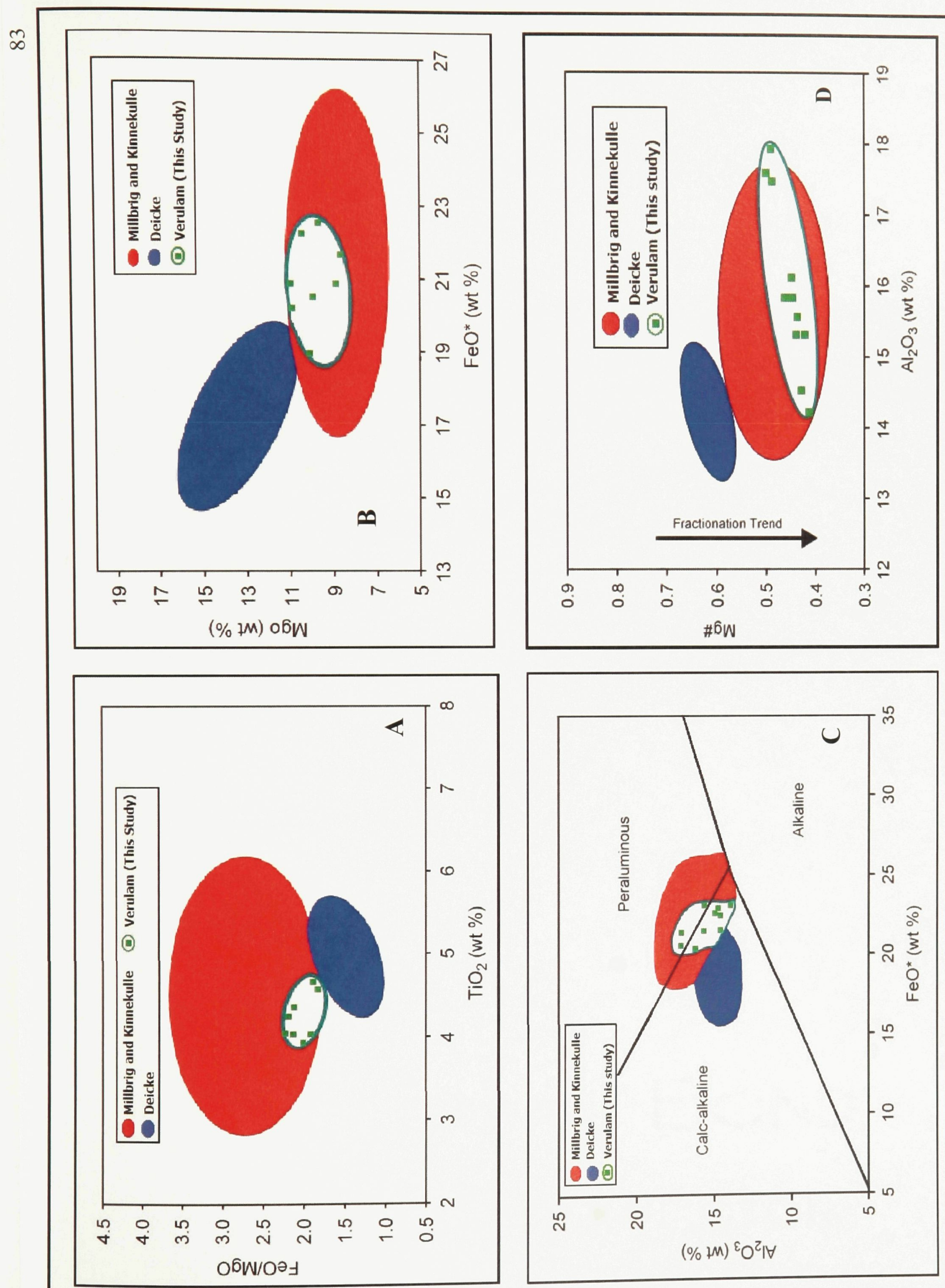
Figure 25. Biotite geochemistry relative to age of volcanic event, Late Ordovician to Early Silurian, peri-Iapetan region. Three groups are defined: Upper Ordovician platform bentonites: I, Deike; II, younger platform deposits; III, Upper Ordovician shale basin and Silurian platform deposits. A) Mica compositions; B) $\mathrm{FeO}$ and $\mathrm{MgO} \%$ according to age; C) $\mathrm{Mg} \#$ vs $\mathrm{MgO} / \mathrm{FeO}$; and D) $\mathrm{K}_{2} \mathrm{O} / \mathrm{CaO}$ (reflecting the extent of alteration) vs $\mathrm{MgO} / \mathrm{FeO}$. 


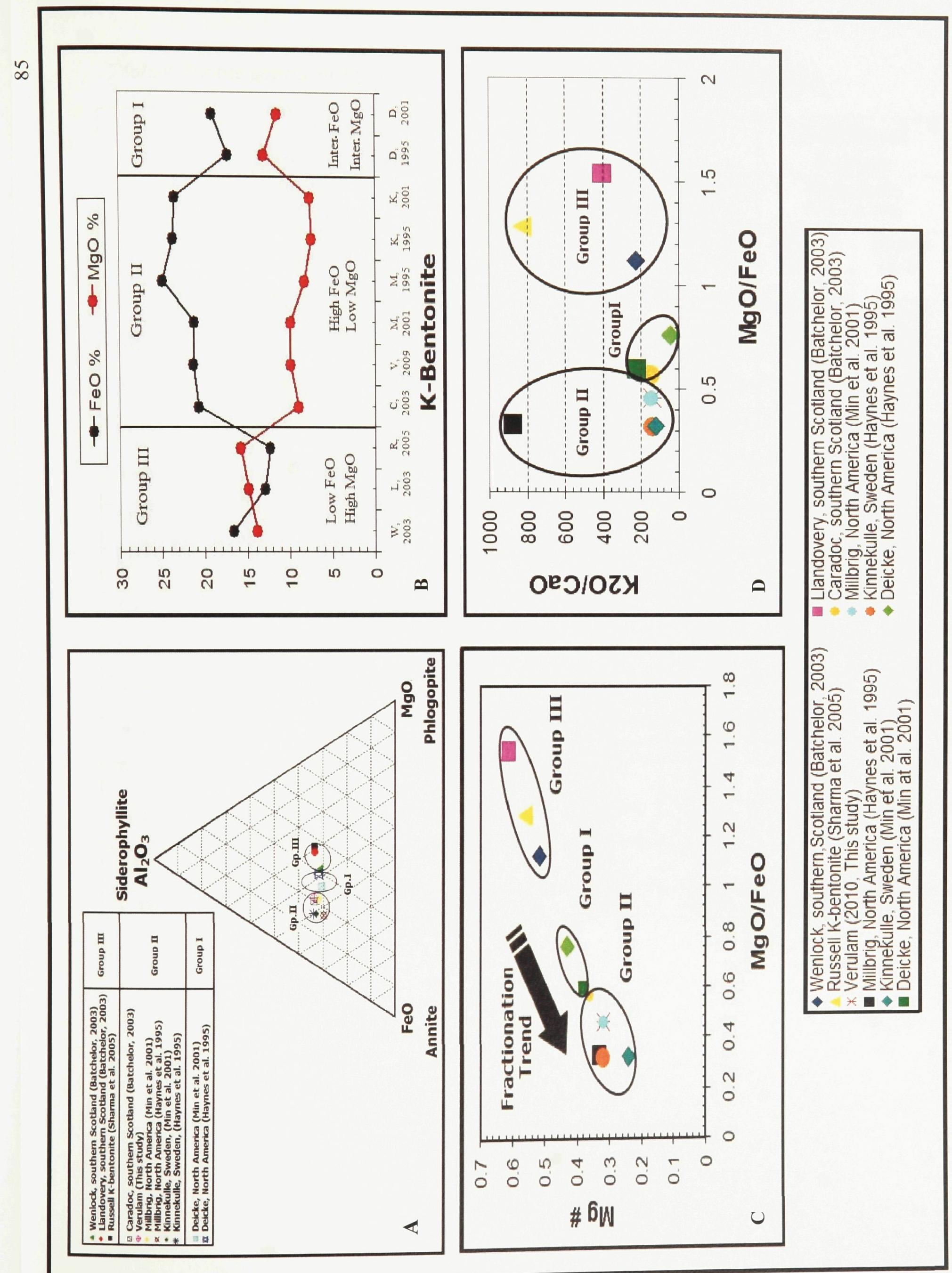


Table 8. Biotite geochemistry of K-bentonite in Canada, North America \& Europe.

\begin{tabular}{|c|c|c|c|c|c|c|c|}
\hline & \multicolumn{3}{|c|}{$\begin{array}{c}\text { Canada } \\
\text { (Eastern Ontario) }\end{array}$} & \multicolumn{2}{|c|}{$\begin{array}{c}\text { eastern North } \\
\text { America }\end{array}$} & \multirow{2}{*}{\multicolumn{2}{|c|}{$\begin{array}{l}\text { Northwest } \\
\text { Europe } \\
\text { Kinnekulle } \\
\text { (Min et al. 2001) }\end{array}$}} \\
\hline & \multicolumn{2}{|c|}{$\begin{array}{l}\text { Verulam Ash } \\
\text { (This Study) }\end{array}$} & \multirow{2}{*}{$\begin{array}{c}\text { Russell } \\
\text { (Sharma et. al. 2005) }\end{array}$} & \multirow{2}{*}{$\begin{array}{c}\begin{array}{c}\text { Deicke } \\
\text { (Min et al. 2001) }\end{array} \\
\begin{array}{c}\text { DK-LQ } \\
(n=8)\end{array}\end{array}$} & \multirow{2}{*}{$\begin{array}{c}\begin{array}{c}\text { Millbrig } \\
\text { (Min et al. } \\
2001\end{array} \\
\begin{array}{c}\text { MB-ST } \\
(n=7)\end{array}\end{array}$} & & \\
\hline & $\begin{array}{c}\text { Russell } \\
(n=6)\end{array}$ & $\begin{array}{c}\text { Billings } \\
\text { Bridge } \\
(n=4)\end{array}$ & & & & $\begin{array}{l}\text { SWE52 } \\
(n=6)\end{array}$ & $\begin{array}{c}\text { DEN22 } \\
(n=3)\end{array}$ \\
\hline & $\begin{array}{c}\text { Average } \\
\text { (S.D.) }\end{array}$ & $\begin{array}{c}\text { Average } \\
\text { (S.D.) }\end{array}$ & $\begin{array}{c}\text { Average } \\
\text { (S.D.) }\end{array}$ & $\begin{array}{c}\text { Average } \\
\text { (S.D.) }\end{array}$ & $\begin{array}{c}\text { Average } \\
\text { (S.D.) }\end{array}$ & $\begin{array}{l}\text { Average. } \\
\text { (S.D.) }\end{array}$ & $\begin{array}{c}\text { Average } \\
\text { (S.D.) }\end{array}$ \\
\hline $\mathrm{SiO}_{2}$ & $\begin{array}{c}35.48 \\
(0.06)\end{array}$ & $\begin{array}{l}\mathbf{3 4 . 7 7} \\
(1.46)\end{array}$ & $\begin{array}{l}36.99 \\
(0.07)\end{array}$ & $\begin{array}{l}\mathbf{3 6 . 0 6} \\
(0.90)\end{array}$ & $\begin{array}{l}\mathbf{3 5 . 5 4} \\
(0.68)\end{array}$ & $\begin{array}{l}\mathbf{3 4 . 4 2} \\
(0.26)\end{array}$ & $\begin{array}{l}\mathbf{3 3 . 8 2} \\
(0.34)\end{array}$ \\
\hline $\mathrm{TiO}_{2}$ & $\begin{array}{l}4.53 \\
(0.14) \\
\end{array}$ & $\begin{array}{l}4.54 \\
(0.02)\end{array}$ & $\begin{array}{l}\mathbf{5 . 4 7} \\
(0.06)\end{array}$ & $\begin{array}{l}\mathbf{4 . 7 5} \\
(0.30) \\
\end{array}$ & $\begin{array}{l}\mathbf{4 . 3 0} \\
(0.37)\end{array}$ & $\begin{array}{l}4.89 \\
(0.48)\end{array}$ & $\begin{array}{l}\mathbf{4 . 1 4} \\
(0.20) \\
\end{array}$ \\
\hline $\mathbf{A l}_{2} \mathbf{O}_{3}$ & $\begin{array}{l}\mathbf{1 5 . 7 2} \\
(0.78)\end{array}$ & $\begin{array}{l}\mathbf{1 6 . 1 6} \\
(0.33)\end{array}$ & $\begin{array}{c}14.17 \\
(0.1)\end{array}$ & $\begin{array}{l}\mathbf{1 3 . 4 8} \\
(0.28) \\
\end{array}$ & $\begin{array}{l}\mathbf{1 4 . 5 7} \\
(1.16)\end{array}$ & $\begin{array}{l}\mathbf{1 5 . 2 4} \\
(0.10)\end{array}$ & $\begin{array}{l}\mathbf{1 5 . 6 8} \\
(0.32)\end{array}$ \\
\hline $\mathrm{FeO}$ & $\begin{array}{l}\mathbf{2 1 . 7 9} \\
(0.07) \\
\end{array}$ & $\begin{array}{l}\mathbf{2 0 . 3 3} \\
(1.19)\end{array}$ & $\begin{array}{c}12.33 \\
(0.2)\end{array}$ & $\begin{array}{l}\mathbf{1 9 . 1 6} \\
(2.05)\end{array}$ & $\begin{array}{l}\mathbf{2 1 . 2 3} \\
(1.85)\end{array}$ & $\begin{array}{l}23.49 \\
(0.45)\end{array}$ & $\begin{array}{l}\mathbf{2 4 . 6 0} \\
(0.12)\end{array}$ \\
\hline $\mathrm{MnO}$ & $\begin{array}{l}\mathbf{0 . 1 6} \\
(0.03) \\
\end{array}$ & $\begin{array}{l}\mathbf{0 . 1 6} \\
(0.04)\end{array}$ & $\begin{array}{l}\mathbf{0 . 1 8} \\
(0.02) \\
\end{array}$ & $\begin{array}{c}\mathbf{0 . 1 8} \\
(0.05) \\
\end{array}$ & $\begin{array}{l}\mathbf{0 . 1 4} \\
(0.05)\end{array}$ & $\begin{array}{l}\mathbf{0 . 2 6} \\
(0.02)\end{array}$ & $\begin{array}{l}\mathbf{0 . 2 9} \\
(0.02)\end{array}$ \\
\hline MgO & $\begin{array}{c}9.7 \\
(0.49)\end{array}$ & $\begin{array}{l}\mathbf{9 . 9 3} \\
(1.07) \\
\end{array}$ & $\begin{array}{l}\mathbf{1 5 . 8 4} \\
(0.12) \\
\end{array}$ & $\begin{array}{l}\mathbf{1 1 . 5 1} \\
(1.18)\end{array}$ & $\begin{array}{r}\mathbf{9 . 8 6} \\
(1.60) \\
\end{array}$ & $\begin{array}{l}7.62 \\
(0.14) \\
\end{array}$ & $\begin{array}{l}7.76 \\
(0.14) \\
\end{array}$ \\
\hline $\mathrm{CaO}$ & $\begin{array}{l}\mathbf{0 . 0 6} \\
(0.05)\end{array}$ & $\begin{array}{l}\mathbf{0 . 0 3} \\
(0.06)\end{array}$ & $\begin{array}{l}\mathbf{0 . 0 1} \\
(0.00)\end{array}$ & $\begin{array}{l}\mathbf{0 . 0 4} \\
(0.04)\end{array}$ & $\begin{array}{l}\mathbf{0 . 0 6} \\
(0.01)\end{array}$ & $\begin{array}{l}\mathbf{0 . 0 7} \\
(0.03)\end{array}$ & $\begin{array}{l}\mathbf{0 . 0 5} \\
(0.00)\end{array}$ \\
\hline $\mathrm{Na}_{2} \mathrm{O}$ & $\begin{array}{r}\mathbf{0 . 2 4} \\
(0.05)\end{array}$ & $\begin{array}{l}0.21 \\
(0.8)\end{array}$ & $\begin{array}{l}\mathbf{0 . 6 1} \\
(0.02)\end{array}$ & $\begin{array}{l}\mathbf{0 . 3 7} \\
(0.03)\end{array}$ & $\begin{array}{l}\mathbf{0 . 4 9} \\
(0.07)\end{array}$ & $\begin{array}{l}\mathbf{0 . 4 5} \\
(0.04)\end{array}$ & $\begin{array}{l}\mathbf{0 . 3 7} \\
(0.07)\end{array}$ \\
\hline $\mathbf{K}_{2} \mathbf{O}$ & $\begin{array}{c}7.08 \\
(0.76) \\
\end{array}$ & $\begin{array}{l}\mathbf{6 . 1 8} \\
(1.05) \\
\end{array}$ & $\begin{array}{r}\mathbf{8 . 2 6} \\
(0.05) \\
\end{array}$ & $\begin{array}{r}\mathbf{8 . 8 7} \\
(0.29) \\
\end{array}$ & $\begin{array}{r}\mathbf{8 . 8 9} \\
(0.17) \\
\end{array}$ & $\begin{array}{r}\mathbf{8 . 5 5} \\
(0.10) \\
\end{array}$ & $\begin{array}{r}8.24 \\
(0.25) \\
\end{array}$ \\
\hline $\mathbf{B a O}$ & $\begin{array}{r}\mathbf{0 . 4 6} \\
(0.25) \\
\end{array}$ & $\begin{array}{r}\mathbf{0 . 3 7} \\
(0.14) \\
\end{array}$ & $\begin{array}{l}\mathbf{1 . 6 4} \\
(0.06)\end{array}$ & $\begin{array}{r}\mathbf{0 . 4 5} \\
(0.18) \\
\end{array}$ & $\begin{array}{l}\mathbf{0 . 4 1} \\
(0.24) \\
\end{array}$ & $\begin{array}{l}\mathbf{0 . 5 6} \\
(0.29) \\
\end{array}$ & $\begin{array}{l}\mathbf{0 . 3 5} \\
(0.08) \\
\end{array}$ \\
\hline $\mathbf{P}_{2} \mathbf{O}_{5}$ & N.A & N.A. & N.A. & $\begin{array}{c}\mathbf{0 . 0 0} \\
(0.01) \\
\end{array}$ & $\begin{array}{c}\mathbf{0 . 0 1} \\
(0.01) \\
\end{array}$ & $\begin{array}{c}\mathbf{0 . 0 0} \\
(0.01)\end{array}$ & $\begin{array}{r}\mathbf{0 . 0 2} \\
(0.02) \\
\end{array}$ \\
\hline $\mathrm{Cl}$ & \begin{tabular}{|l|}
$\mathbf{0 . 5 1}$ \\
$(0.01)$ \\
\end{tabular} & $\begin{array}{r}\mathbf{0 . 0 8} \\
(0.01) \\
\end{array}$ & \begin{tabular}{|c|}
$\mathbf{0 . 0 3}$ \\
$(0.00)$ \\
\end{tabular} & \begin{tabular}{|l}
$\mathbf{0 . 1 4}$ \\
$(0.07)$ \\
\end{tabular} & $\begin{array}{c}\mathbf{0 . 1 1} \\
(0.03) \\
\end{array}$ & $\begin{array}{l}\mathbf{0 . 2 0} \\
(0.01) \\
\end{array}$ & $\begin{array}{r}\mathbf{0 . 2 2} \\
(0.02) \\
\end{array}$ \\
\hline $\mathbf{F}$ & $\begin{array}{c}\mathbf{0 . 1 1} \\
(0.04) \\
\end{array}$ & $\begin{array}{l}\mathbf{0 . 6 6} \\
(0.11)\end{array}$ & $\begin{array}{c}\mathbf{0 . 9 6} \\
(0.02) \\
\end{array}$ & \begin{tabular}{|c|}
$\mathbf{0 . 5 9}$ \\
$(0.18)$ \\
\end{tabular} & $\begin{array}{c}\mathbf{0 . 7 6} \\
(0.19) \\
\end{array}$ & $\begin{array}{r}\mathbf{0 . 7 5} \\
(0.06) \\
\end{array}$ & \begin{tabular}{|l|}
$\mathbf{0 . 5 8}$ \\
$(0.09)$ \\
\end{tabular} \\
\hline Total & 95.84 & 93.46 & 96.50 & 95.61 & 96.38 & 96.50 & 96.13 \\
\hline
\end{tabular}




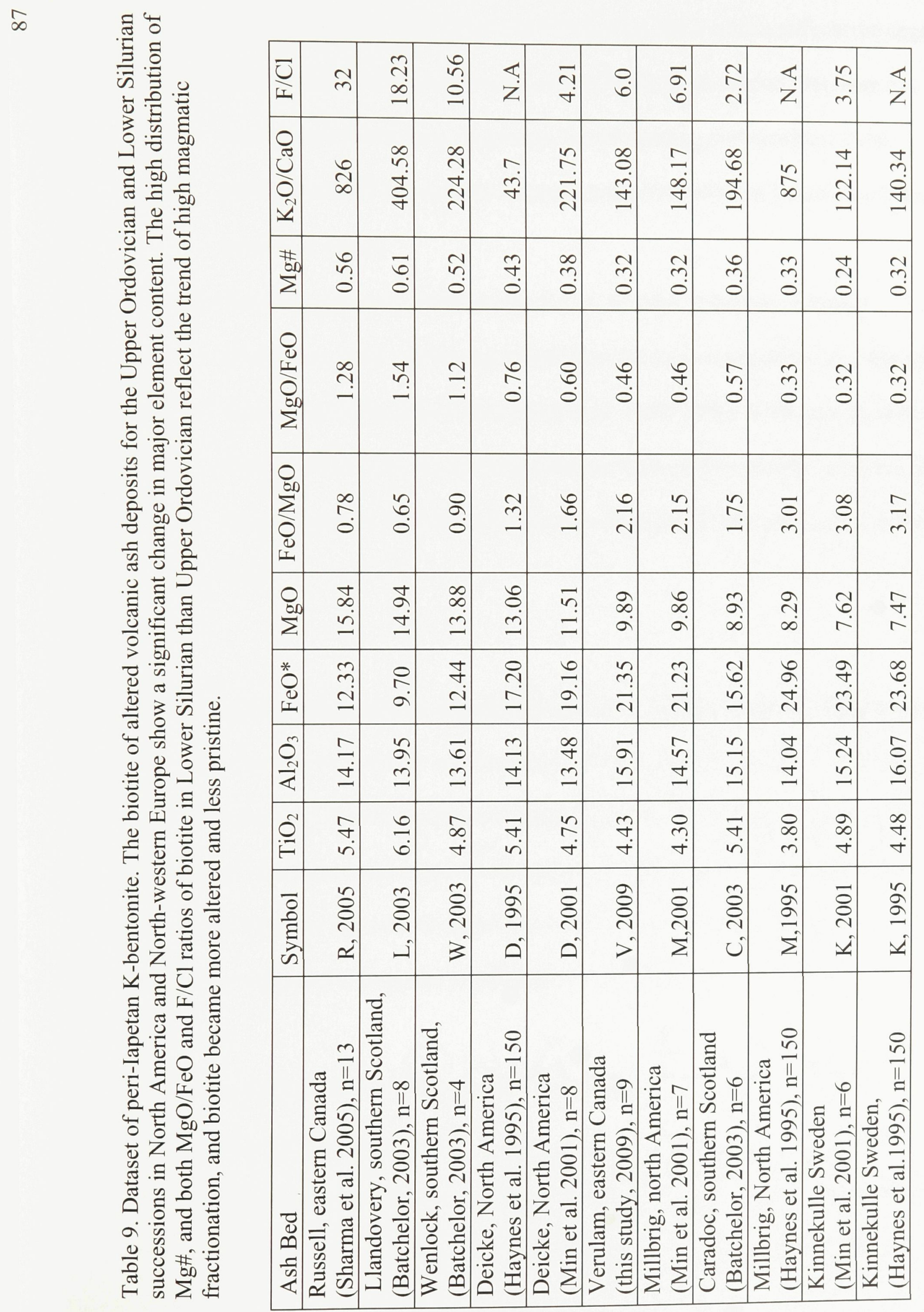


c) Evaluation of the $\mathrm{Mg} \#(\mathrm{Mg} /(\mathrm{Mg}+\mathrm{Fe}))(\mathrm{Fig}$. 25C), which reflects the degree of magma fractionation (Haynes, 1995; Huff, 2008), shows that there is a good separation among the groups, but illustrating that, combined, the platform-hosted volcanic ash deposits were derived from a more fractionated magmatic source.

d) Through increased alteration the $\mathrm{K}_{2} \mathrm{O} / \mathrm{CaO}$ ratio of biotite decreases (Batchelor, 2003), reflecting a shift from the ideal composition accompanying the particular $\mathrm{MgO} / \mathrm{FeO}$ ratio (Huff et al. 1992; 2008). With exception of one dataset $(n=150)$ for the Millbrig (Haynes et al., 1995), all other samples fall below a ratio of 400 , with slightly more than half below a ratio of 200 (Fig. 25D).

\section{New tectonostratigraphic significance of Late Ordovician volcanism}

Most studies on volcanic ash deposits in the Ordovician and Lower Silurian have focussed on the use of biotite and geochemical proxies to help identify magmatic source and extent of volcanism, and temporal correlation of geographically separate events.

Study of the Verulam deposits reveal two new discoveries related to Late Ordovician platform development in response to tectonics and volcanism bordering the Iapetan Ocean basin.

Step-back in the Laurentian platform margin. Subsurface correlation extending from the Michigan Basin through to southern Quebec (Fig. 9), based on incorporating previous correlations of Kolata et al. (1996) and Bergström and Mitchell (1994) with the Russell core (this study), reveals that the Verulam volcanic event 
coincides with a prominent regression of the Laurentian margin within the Quebec Reentrant, as preserved near Quebec City (Fig. 9). This coincidece would appear to identify increased subsidence within the foreland basin contemporaneous with volcanic eruption. Joy et al. (2000) have documented abrupt changes in stages of subsidence rates associated with Trenton age platform and related basin sedimentation along the eastern Laurentian margin.

Magmatic stages of volcanic arc evolution. There appears to be a prominent stratigraphic grouping of $\mathrm{Mg} \#$ derived from the altered volcanic ash deposits in the Upper Ordovician-Lower Silurian succession (Fig. 26). These groupings are similar to the three groups defined in Fig. 25, and show that the Chatfieldian volcanism represented by the Millbrig and Kinnekulle deposits was possibly more fractionated than that of the Diecke event, but strongly fractionated relative to the bentonite in the post-platform Taconic shale basin (Sharma et al., 2005) and younger Silurian events.

Sharma et al. (2005) had recognized a distinct difference in $\mathrm{MgO}$ vs $\mathrm{FeO}$ with those of volcanics in the older platform carbonates, and had proposed that this may signal a change in the volcanic arc development coincident with platform foundering and formation of the Taconic deep-water shale basins. Incorporating more recent data, including this study, now appears to identify descrete time periods of different stages of volcanism. The position of the Chatfieldian-Turinian boundary is currently debated (Fig. 26): traditionally, it was placed between the Millbrig and Deicke volcanic events, but recent revision (Brett et al., 2004) places it above the Millbrig event. Distribution of Mg\# support the latter, the Millbrig / Kinnekulle and Verulam eruptive events have similar Mg\#. This suggests a corresponding change in the magmatic evolution of the orogen. 
Figure 26. Magmatic stages of volcanic arc evolution according to changing $\mathrm{Mg \#}$ associated with micas in volcanic events along the Laurentian continental margin. Chatfieldian volcanism represented by the Millbrig, Kinnekulle, and Verulam deposits was possibly more fractionated than that of the Deicke event, but more so in comparison to the events after platform collapse that produced the Taconic shale basin (Sharma et al., 2005 ) as well as younger Silurian events. 


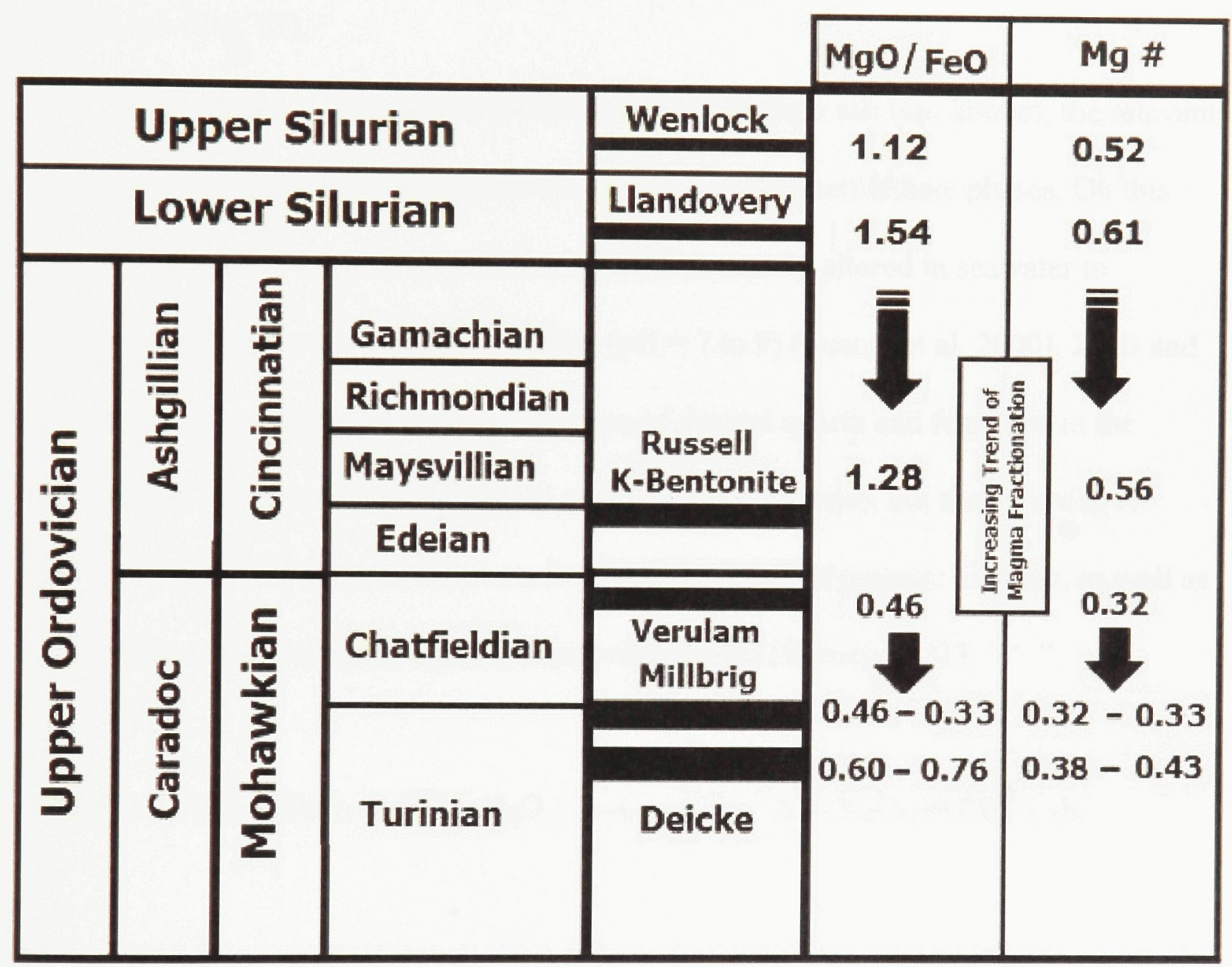




\section{DIAGENESIS OF VOLCANIC ASH, VERULAM FORMATION}

Volcanic ash is highly reactive within the marine and burial environments such that alteration is usually rapid. One of the most common end-products is clay. The type of clay is a function of magma composition and primary minerals associated with the derived ash (Fig. 27).

With regard to the postulated source of the Verulam ash (see above), the relevant clay mineral products will be restricted to the acidic and intermediate phases. On this basis, primary glass associated with the ash will be rapidly altered in seawater to montmorillonite (most commonly) or illite ( $\mathrm{pH}=7$ to 9) (Fuente et al. 2000). XRD and microscopy demonstrate an apparent absence of detrital quartz and feldspars in the Verulam ash deposits (supports high-Si andesite or rhyodacite), but the presence of kaolinite. This latter mineral can form from the alteration of potassic feldspar, as well as the alteration of muscovite (1) and montmorillonite (2) (Berner, 1971):

${ }_{2} \mathrm{KAl}_{2}\left(\mathrm{SiO}_{3} \mathrm{AlO}_{10}\right)(\mathrm{OH})_{2}+{ }_{2} \mathrm{H}^{+}+{ }_{3} \mathrm{H}_{2} \mathrm{O} \longrightarrow \mathrm{Al}_{4}\left(\mathrm{Si}_{4} \mathrm{O}_{10}\right)(\mathrm{OH})_{8}+{ }_{2} \mathrm{~K}^{+}$

${ }_{4} \mathrm{NaAlMgSi}_{4} \mathrm{O}_{8}(\mathrm{OH})_{2}+{ }_{6} \mathrm{H}_{2} \mathrm{CO}_{3} \longrightarrow{ }_{3} \mathrm{Al}_{4} \mathrm{Si}_{4} \mathrm{O}_{8}(\mathrm{OH})_{4}+{ }_{2} \mathrm{Mg}^{2+}+{ }_{2} \mathrm{Na}+{ }_{6} \mathrm{HCO}_{3}+{ }_{10} \mathrm{H}_{4} \mathrm{SiO}_{4}$

Once formed, broad clay mineralogical transformations occur with burial.

Montmorillonite changes to $\mathrm{I} / \mathrm{S}$ combinations over the range of $50-80^{\circ} \mathrm{C}$; then, the 
Figure 27 Clay mineral products relative to rock types and magma source. 


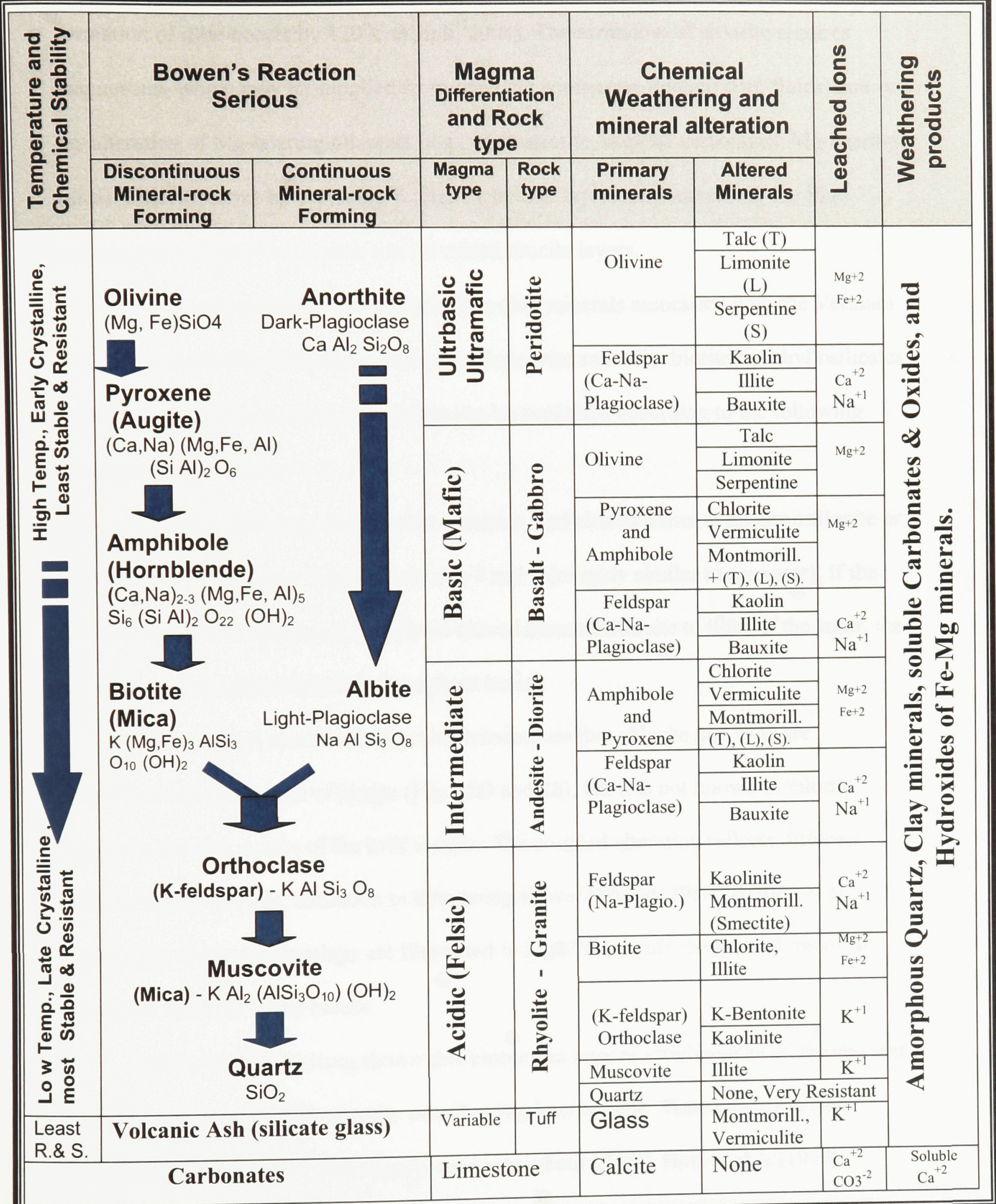


formation of illite occurs by $120^{\circ} \mathrm{C}$ (Kiipli, 2004). The formation of chlorite requires magnesium, which may be supplied from seawater (or marine-derived pore fluids) and-or the alteration of Mg-bearing minerals (e.g., Mg-smectite, skeletal carbonates, Mg-bearing mica). Chlorite forms by replacing $\mathrm{K}$ sites by brucite layers, and increasing the $\mathrm{Mg}$ concentration in octahedral sites and the added brucite layers.

$\mathrm{XRD}$ analysis shows that the principle clay minerals associated with the Verulam are illite and chlorite, with minor kaolinite. Muscovite and relict biotite are phyllosilicates present as well. This mineral assemblage can be explained according to the following paragenetic succession:

Volcanic glass was deposited in seawater, and altered either to montmorillonite or illite; the latter can occur at $\mathrm{pH}$ of between 7 and 9 (roughly similar to seawater). If the former, then burial diagenesis completed altered montmorillonite to illite. If the latter, the mineralogy was relatively stable throughout burial.

The Verulam altered ash deposits demonstrate that chlorite and illite are associated with alteration of biotite (Fig. 15D and 28), but it is not known if chlorite occurs in the clay matrix of the bulk sample. The coupled alteration reflects different rates of transformation, alteration to illite being slower (Morad, 1984; Weaver et al., 1984). The above relationships are illustrated in Fig. 28 showing the general trends of alteration from the initial biotite.

Huff et al. (1997) have shown that biotite can alter to interlamellae of chlorite and smectite, presumably with smectite transforming later to illite. Transformation from mixed (I/S) layer clays to illite usually occurs by about $120^{\circ} \mathrm{C}$. Huff et al.'s (1997) paragenetic history supports chlorite alteration at temperatures below this limit. Iijima 
(1978) found diagenetic chlorite in volcanic ash deposits associated with burial temperatures of between $90^{\circ}$ and $120^{\circ} \mathrm{C}$, and Velde (1985) reports diagenetic chlorite forming at temperatures between $80^{\circ}$ and $160^{\circ} \mathrm{C}$.

The thermal history of the Ottawa Embayment offers some constraints on thermal conditions for chlorite development. Work by Legall et al. (1981) showed that conodont alteration indices of 3.0 are associated with Upper Ordovician limestones within, and to the southeast of, the City of Ottawa. This translates into a burial history that subjected the platform carbonates to maximum burial temperatures of $\sim 110$ to $120^{\circ} \mathrm{C}$. Dix and Robinson (2003) demonstrated that local hydrothermal mineralization in the Ottawa Embayment achieved temperatures of $>180^{\circ} \mathrm{C}$, but there is no evidence for such influence in the vicinity of the Russell or Billings Bridge sites. In summary, further study of the chlorite is needed, and previous authors dealing with Ordovician volcanic ash deposits have not addressed the biotite-to-chlorite reactions. 
Figure 28 Chlorite and illite formation by alteration of biotite. A, B, and C) Chlorite and illite form from biotite alteration, with the diagenetic trends illustrated by arrows. D) General compositional areas for the three minerals showing why biotite is preferentially easily altered to chlorite rather than illite. 


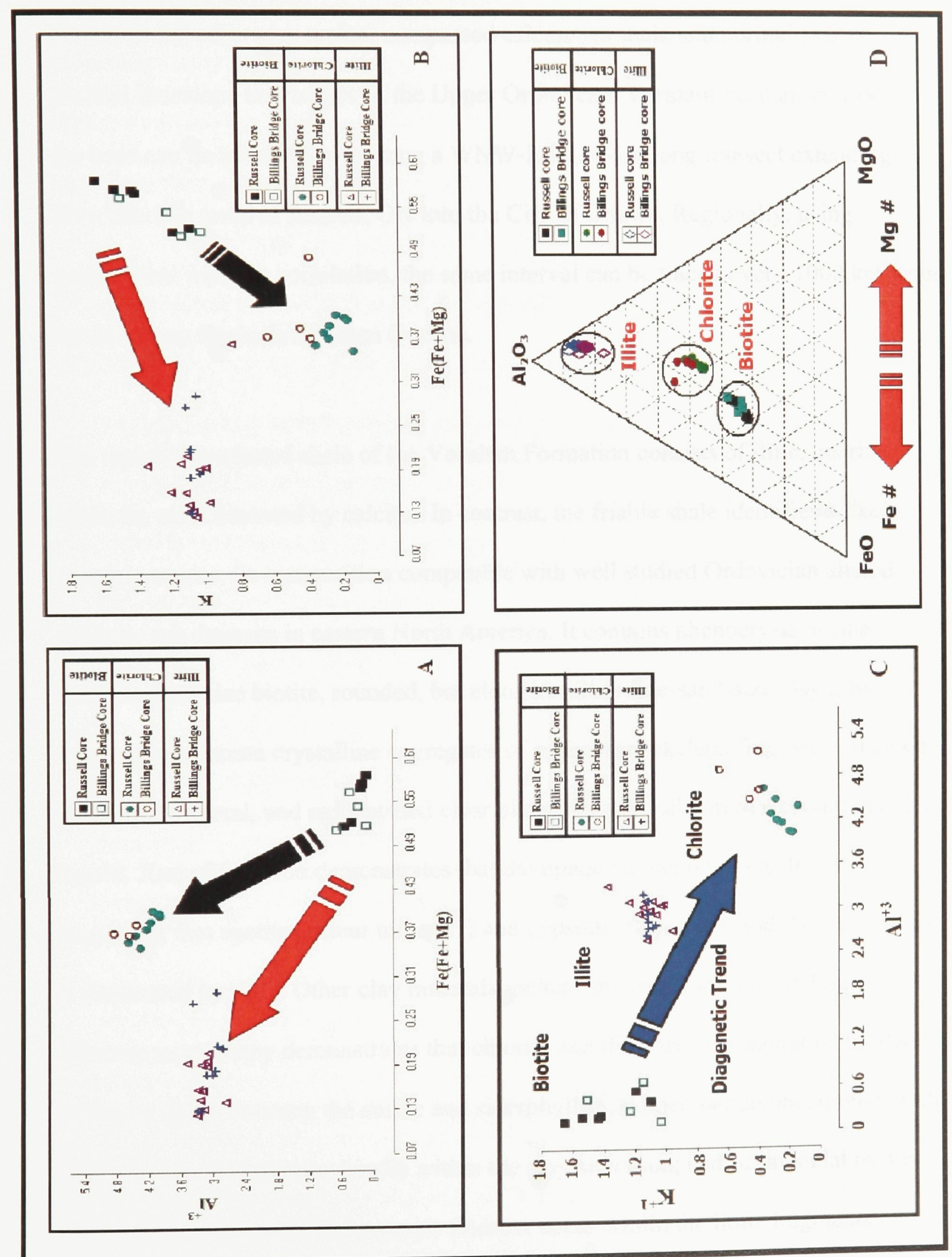




\section{CONCLUSIONS}

1- Two anomalous (friable) thin ( $\mathrm{cm}$-scale) biotite-bearing shales form a couplet that punctuate the otherwise typical compacted calcareous shale and normal-marine skeletal limestone that makes of the Upper Ordovician Verulam Formation. Locally, the beds can be traced in core along a WNW-ESE 24-km long transect extending from near the town of Russell, ON into the City of Ottawa. Regionally, using geophysical well log correlation, the same interval can be traced over $\sim 1000 \mathrm{~km}$ from the Michigan Basin to southern Quebec.

2- The typical compacted shale of the Verulam Formation consists of illite, quartz, kaolinite, and cemented by calcite. In contrast, the friable shale identifies a likely volcanic source, its composition compatible with well studied Ordovician altered volcanic ash deposits in eastern North America. It contains phenocrysts of fine- to medium sand-size biotite, rounded, but elongate silt to fine-sand-size clay lithic fragments, common crystalline aggregates of pyrite, rare skeletal fragments of calcite, an opaque mineral, and unidentified clear platey crystals, all within a crystalline clay matrix. Xray diffraction demonstrates that the opaque mineral is most likely magnetite, that apatite (=clear mineral?) and gypsum are present, and the clay matrix is dominated by illite. Other clay minerals include chlorite, kaolinite, and muscovite. Electron microscopy demonstrates that chlorite and illite are both alteration products of biotite (lying between the annite and siderphyllite endmember compositions), with only thin lamellae of relict biotite within the clay alteration; and, framboidal pyrite and fragments of quartz and potassic feldspar occur within the lithic fragments 
otherwise dominated by illite.

3- Use of standard geochemical proxies to type composition and tectonic setting of the magmatic source reveals a trachyandesite to rhyodacite/dacite source fitting a calcalkaline volcanic arc suite associated with a destructive (e.g., subduction-related) plate boundary. This is supported by lack of quartz, thus less evolved than rhyolite.

4- Within the framework of volcanic ash deposition, several observations carry extra significance:

i) assuming that airfall transport within a volcanic ash plume is reasonably unidirectional, a noted decrease in grain size of altered biotite phenocrysts from 300-400 microns to 50-75 microns over the $\sim 24-\mathrm{km}$ distance from near Russell, ON, to the City of Ottawa, may identify a source positioned to the ESE within the volcanic arc that lay along the developing foreland basin along eastern Laurentia;

ii) absence of any altered ash bed interval only $3 \mathrm{~km}$ northwest of its presence in the City of Ottawa may identify erosion of the once deposited ash, or depositional controls such as wave or current agitation preventing deposition;

iii) The observed clay-dominated lithic fragments may represent altered fragments of pumice or congealed magma transported during eruption. The measured aspect (length/width: L/W) ratios for the lower altered ash bed at the two core sites show an apparent increase in variation in the NW-directed decrease in grain size; $\sim 1.75$ 
\pm 0.54 to $2.24 \pm 1.46$, respectively. The upper altered ash bed to the SE has a similar ratio as the older bed, but no material was available to compare moving NW along the transect.

5- The predominance of illite within the altered ash beds is compatible with the known burial history of the Ottawa Embayment wherein burial temperatures reached at least $120^{\circ} \mathrm{C}$. Formation of chlorite as an alteration product of biotite may be more problematic although published studies place the temperature range of diagenetic chlorite (in absence of biotite) as 90 to $160^{\circ} \mathrm{C}$.

6- Attempt to determine the age of the biotite from Ar-Ar analysis failed due to too extensive alteration by chlorite and illite.

7- Placed in the regional framework of Late Ordovician volcanism within the periIapetan (eastern Laurentia, western Baltica) region, a successive stratigraphic change in the Mg\# of altered ash deposits moving from the base of the Late Ordovician (Chatfieldian Stage; 457 Ma) to the Early Silurian (Wenlock Stage; 425 Ma) identifies decrease in magmatic fractionation with the greatest change appear to occur coincident with regional foundering of the foreland carbonate platform and development of deep-water shale basins. 


\section{REFREFRENCE}

Abdel-Rahman, A. (1994) nature of Biotite from Alkaline, Calc-alkaline, and Peraluminous Magmas. Journal of Geology, Vol. 35, Part 2, pp.525-541.

Bailey, S.W. (1984) Classification and structure of micas. Crystal chemistry the true micas. Reviews in Mineralogy, Min. Soc. America., 13, 1-60.

Batchelor, R.A. (2003) Geochemistry of biotite in metabentonites as an age discriminant, indicator of regional magma sources and potential correlating tool. Mineralogical Magazine; Vol. 67; no. 4; p. 807-817.

Bellanca, A., Massetti, D., Neri, R. and Venezia, F. (1999) Geochemical and Sedimentological evidence of productivity cycles recorded in Toarcian Black shales from the Belluno Basin, Southern Alps, Nothern Italy. J Sedimet Research, 69: 466-476.

Bergström, S.M, Huff, W.D., Saltzman, M.R., Kolata, D.R., and Leslie, S.A. (2004) Trans-Atlantic relations of the Ordovician Millbrig and Kinnekulle K-bentonites. The sedimentary Records, Vol. 2(4).

Bergström, S.M, and Mitchell, C.E. (1994) Regional relationships between Middle and early Late Ordovician standard succession in New York and Quebec and the Cincinnati region in Ohio, Indiana, and Kentucky. In Studies in stratigraphy and paleontology in honor of Donald W. Fisher (ed. E. Landing), pp.5-20. New York State Museum Bulletin 481.

Berkley J., and Baird G. (2002) Calcareous K-bentonite deposits in the Utica Shale and Trenton Group (Middle Ordovician), of the Mohawk Valley, New York State. Physics and chemistry of the Earth; 27, p.265-278.

Berner, R. (1971) Principles of chemical sedimentology. Publish, McGraw-Hill Book Co., 300 PP.

Brand, U., and Veizer, J., 1981, Chemical diagenesis of a multicomponent carbonate (system-1) Trace elements: Journal of Sedimentary petrology, V.50, p.1219-1236.

Brazier, S., Sparks, R.S.J., Carey, S.N., Sigurdsson, H., and Westgate, J.A. (1983) Bimodal grain size distribution and secondary thickening in air-fall ash layers. Nature, v. 301, p. 115-119.

Brett, C.E., McLaughlin, P.I., Cornell, S.R., and Baird, G. (2004) Comparative sequence stratigraphy of two classic Upper Ordovician succession, Trenton Shelf (New York-Ontario) and Lexington Platform (Kentucky - Ohio): implications for eustasy 
and local tectonism in eastern Laurentia. Palaeogeography, Palaeoclimatology, Palaeoecology, v. 210, p. 295-329.

Brumsack, H. J. (1989) Geochemistry of recent TOC-rich sediments from the Gulf of California:Geologische Rundschau, v.78, pp.851-882.

Calvert, S. E. (1976) The mineralogy and geochemistry of nearshore sediments. In: J.P. Riley and R. Chester, Editors, Chemical Oceangeography, University Press, New York: $187-280$.

Carey, S. (1997) influence of convective sedimentation on the formation of widespread tephra fall layers in the deep sea. Geology, v. 25; no.9; p.839; 4 figures.

Deer, W.A., Howie, r. A., and Zussman, J. (1992) An introduction to the rock-forming minerals. Longman Scientific \& Technical, $\left(2^{\text {nd }}\right.$ ed. $)$, p. 696.

Dix, G. and Al Rodhan, Z. A. (2006) New geological framework for the Middle Ordovician Carillon Formation (uppermost Beekmantown Group, Ottawa Embayment): onset of Taconic foreland deposition and tectonism within the Laurentian platform interior. Can. J. Earth Sci.; 1376-1387.

Dix, G.R., and Robinson, G.W. (2003) The geochemical record of hydrothermal mineralization and tectonism inboard of the Appalachian Orogeny: the Ottawa Embayment. Chemical Geology, 197:29-53.

Fuente, S., Cuadros, S. and Linares, J. (2000) Electronic Microscopy study of the volcanic tuff alteration to illite-smectite under hydrothermal conditions. Clays and Clay Minerals, Vol. 48, 3, pp. 339-350.

Giudotti, C. V. (1984) Micas in metamorphic rocks. Review in mineralogy, Min. Soc. America, 357-67.

Haynes, G.T., Melson and Kunk, M.J. (1995) W.G., and Composition of biotite phenocrysts in Ordovician tephras casts doubt on the proposed trans-Atlantic correlation of the Millbrig K-bentonite (United States) and the Kinnekulle K-bentonite (Sweden). Geology; v. 23; no. $9 ;$ p. $847-850$.

Huff, W.D. (2008) Ordovician K-bentonite: Issues in interpreting and correlating ancient tephras. Quaternary International, 178, PP. 276-287.

Huff, Warren D., Bergstrom, S. and Kolata, D. (1992) Gigantic Ordovician volcanic ash fall in North America and Europe, biological tectonomagmatic, and eventstratigraphic significance: Geology, v. 20, p. 875. 
Huff, Warren D., Bergstrom, S., Kolata, D. and Sun, H. (1997) The Lower Silurian Osmundsberg K-bentonite. Part II: mineralogy, geochemistry, chemostratigraphy and tectonomagmatic significance. Geol. Mag. (135)(1), pp.15-26.

Iijima, A. (1978) Geological occurrences of zeolite in marine environments. In: L.B. Sand and F.A. Mumpton (Eds) Natural Zeolites: Occurrence, Use, Properties, Pergamon Press, New York, pp. 175-198.

Joy, M.P., Mitchell, C.E., and Adhja, S., (2000) Evidence of a tectonically driven sequence succession in the Middle Ordovician Taconic foredeep. Geology, v. 28, p. 727-730.

Kaufmann, A.J. and Knoll, A.H. (1995) Neoproterozoic variation in the C-isotopic composition of seawater biogeochemical implication: Precambrian Research, Vol. 73, p.27-49.

Kay, G.M. (1931) Stratigraphy of the Ordovician Hounsfield metabentonite. Journal of Geology 29 (1931), pp. 361-376.

Kay, G.M. (1935) Distribution of Ordovician altered volcanic materials and related clays. Geological Society of America, Bulletin 46 (1935), pp. 225-244.

Kay, G.M. (1942) Ottawa-Bonnechère graben and Lake Ontario homecline. Geological Survey of America Bulletin, 53:585-646.

Kiernan, J.P. (1999) Lithistratigraphy, sedimentology and diagenesis of the Upper Ordovician Hull Beds and Verulam formation, Upper Ottawa Group, eastern Ontario. Master thesis, Carleton University.

Kiipli, T., Kallaste, T., and Kiipli, E. (2004) Areal Variations in the composition of the Kinnekulle altered volcanic ash bed (Caradocian)-relations to sedimentary. WOGOGOB, 2004, pp.55-56.

Kolata, D.R., Huff, W.D., and Bergström, S. M. (1996) Ordovician K-bentonite of eastern North America: Geological Society of America special paper 313, 84p.

Kumerapelli, P.S. (1985) Vestiges of Iapetun rifting in the craton west of northern Appalachians. Geosciences Canada, 12:54-59.

Kumerapelli, P.S., and Saull, V.A. (1966) The St. Lawrence Valley System: a North American equivalent of the East African Rift Valley System. Canadian Journal Earth Sciences, 3:639-658.

Leat, P. T., Jackson, S.E., Thorpe, R.S. \& Stillman, C.J. (1986) Geochemistry of bimodal basalt-subalkaline/peralkaline rhyolite provinces within the southern british Caledonides. Journal of the Geological Society, London 143, 259-73. 
Legall, F.D., Barnes, C.R., and Macqueen, R.W. (1981) Thermal maturation, burial history and hotspot development, Paleozoic strata of southern OntarioQuebec, from conodont and acritarch colour alteration studies: Bulletin of Canadian Petroleum Geology, v. 29, p. 492-539.

Liberty, B.A. (1969) Paleozoic geology of the Lake Simoce area, Ontario. Geological Survey of Canada, Memoir 335.

McLennan, S.M., Hemming, S., McDaniel, D.K. and Hanson, G.N. (1993) Geochemical approaches to sedimenatation, provenance and tectonics. Geol Soc Am Spec Paper 285: 21-40.

Mereu, R.F., Wang, D., Kuhn, O., Forsyth, D.A., Green, A. G., Morel, P., Buchbinder, G.G.R., Crossely, D., Schwarz, E., Duberger, R., Brooks, C. \& Clowes, R. (1986) the 1982 COCRUST seismic experiment across the Ottawa-Bennechere graben and Grenville Front in Ontario and Quebec. Geoph. J. of the Royal Astron. Soc. 84, 491-514.

Min, K. Renne, P.R., and Huff, W.D. (2001) 40Ar/39Ar dating of Ordovician Kbentonite in Laurentia and Baltoscandia: Earth and Planetary Science Letters, v. 185, pp.121-134.

Morad, S. (1983) Diagenetic matrix in Proterozoic greywackes from Sweden. $J$. Sediment. Petrol., 53, pp.51-56.

Norrish, K. (1969) An accurate, speetrographic method for the analysis of a wide range of geological samples. Geochim. Cosmochim. Acta 33 (1969), pp. 431-453.

Ontario Geological Survey, City of Ottawa, based on www.ottawamap.com.

Pearce, J.A., Harris, N.B. and Tindle, A.G. (1984) Trace elements discrimination diagrams of the tectonic interpretation of granitic rocks. Journal of Petrology, 25, pp. 956-83.

Riley, C. M., Rose, W. I. and Bluth, G.J. (2003) Quantitative shape measurements of distal volcanic ash. Journal of Geophysical Research; Vol.108, no. B10, 2504.

Rollinson, H. (1995) Using geochemical data: evaluation, presentation, interpretation. Longman Group, UK. P.352.

Romando, R.E. and Benn, K. (2005) Evolution of faulting and paleo-stress field within the Ottawa graben, Canada Journal of Geodynamics, 39:337-360.

Salad Hersi, S, and Dix, G., (1999( Blackreivean (lower Mohawkian, upper Ordovician) litholostratigrahpy, rhythmicity, and paleogeography: Ottawa Embayment, Eastern Ontarion, Canada. Can. J. Earth Science, 36:2033-2055. 
Sanford, B. (1993a) St. Lawrence platform-geology. In sedimentary cover of the craton in Canada, Geology of Canada. Edited by D.F. Stott and J.D. Aitken., no.5. pp.723786.

Sharma, S., Dix, G. and Villeneuve, M. (2005) Petrology and potential tectonic significance of K-bentonite in a Taconic shale basin (eastern Ontario, Canada), northern Appalachians. Geol. Mag. 142(2), pp. 145-158.

Sharma, S., Dix, G. and Riva, JF.V. (2003) LateOrdovician platform foundering, its paleoceangraphy and burial, as preserved in separate (eastern Michigan, Ottawa Embayment) basins, southern Ontario: Canadian Journal of Earth Sciences, v. 40, pp.135-148.

Shields, G., Carden, G., Veizer, J. Meidla, T., Rong, J., and Yu Li, R. (2003). Sr, C, and O isotope geochemistry of Ordovician brachipods: A major isotopic event around the Middle-Late Ordovician transition. Geochimica et Cosmochimica Acta, Vol. 67, No.11, pp. 2005-2025.

Sloss, L.L. (1963) Sequences in the Craton of North America. Geological Society of America Bulletin. 74:93-114.

Trevail, R.A. (1990) Ordovician K-Bentonite in the Subsurface of South-western Ontario. In Proceedings, Ontraio Petroleum Institution conference 29 $9^{\text {th }}$ Annual Conference: Ontario Petroleum Institute, 18p.

Truax, T. (2008) Facies and stratigraphy of the Hull and Verulam Formations (Upper Ordovician) as defined from the Russell Core, eastern Ontario. Unpublished B.Sc. thesis, Carleton University, $51 \mathrm{pp}$.

Velde, B. (1985) Clay minerals: A physico-chemical explanation of their occurrence. Elsevier, PP. 427.

Weaver, C.E, Highsmith, P.H, and Wampler, J.M. (1984) Shale-Slate Metamorphism in Southern Appalachians. Developments in Petrology, 10, Elsevier, 235pp.

Williams, D.A. (1991) Paleozoic geology of Ottawa-St. Lawerence Lowlands, southern Ontario. Ontario Geological Survey, Open File Report 5770.

Winchester, J.A. and Floyd, P.A. (1977) Geochemical discrimination of different magma series and their differentiation products using immobile elements. Chem. Geol., 20, $325-43$.

Wood, D. (1980) The application of Th-Hf-Ta diagram to problems of tectonomagmatic classification and to establishing the nature of crustal contamination of basaltic lavas of the British Tertiary Volcanic Province. Earth and Planetary Science Letters, 50, pp.11-30. 
APPENDIX A : Length (L), width (W), and L/W ratios of lithic fragments in Upper ash bed, Russell core

1. Upper friable shale, Russell core, $n=150$

$\begin{array}{cccc}\text { Frequency } & \text { Length } & \text { Width } & \text { L/W Ratio } \\ 1 & 0.832 & 0.391 & 2.13 \\ 2 & 0.514 & 0.248 & 2.07 \\ 3 & 0.778 & 0.438 & 1.78 \\ 4 & 0.336 & 0.248 & 1.35 \\ 5 & 0.428 & 0.137 & 3.12 \\ 6 & 0.459 & 0.333 & 1.38 \\ 7 & 0.68 & 0.405 & 1.68 \\ 8 & 0.535 & 0.26 & 2.06 \\ 9 & 0.758 & 0.354 & 2.14 \\ 10 & 0.553 & 0.392 & 1.41 \\ 11 & 0.397 & 0.342 & 1.16 \\ 12 & 0.483 & 0.28 & 1.72 \\ 13 & 0.452 & 0.329 & 1.37 \\ 14 & 0.504 & 0.214 & 2.36 \\ 15 & 0.373 & 0.317 & 1.18 \\ 16 & 0.631 & 0.817 & 0.77 \\ 17 & 0.638 & 0.331 & 1.93 \\ 18 & 0.315 & 0.344 & 0.92 \\ 19 & 0.826 & 0.465 & 1.78 \\ 20 & 0.546 & 0.399 & 1.37 \\ 21 & 0.92 & 0.695 & 1.32 \\ 22 & 0.342 & 0.228 & 1.50 \\ 23 & 0.61 & 0.406 & 1.50 \\ 24 & 0.658 & 0.292 & 2.25 \\ 25 & 0.851 & 0.398 & 2.14 \\ 26 & 0.65 & 0.31 & 2.10 \\ 27 & 0.468 & 0.238 & 1.97 \\ 28 & 0.619 & 0.29 & 2.13 \\ 29 & 0.69 & 0.375 & 1.84 \\ 30 & 1.395 & 0.503 & 2.77 \\ 31 & 0.459 & 0.269 & 1.71 \\ 32 & 0.483 & 0.304 & 1.59 \\ 33 & 1.152 & 0.723 & 1.59 \\ 34 & 0.713 & 0.484 & 1.47 \\ 35 & 0.482 & 0.376 & 1.28 \\ 36 & 0.542 & 0.575 & 0.94 \\ 37 & 0.635 & 0.229 & 2.77 \\ 38 & 1.021 & 0.475 & 2.15 \\ 39 & 0.797 & 0.578 & 1.39 \\ 40 & 0.666 & 0.286 & 2.32 \\ 41 & 0.351 & 0.658 & 0.53 \\ 42 & 0.264 & 0.703 & 0.38 \\ 43 & 0.317 & 0.545 & 0.58 \\ & & & \end{array}$




\begin{tabular}{lccc}
44 & 0.322 & 0.65 & 0.49 \\
45 & 0.231 & 0.678 & 0.34 \\
46 & 0.54 & 0.497 & 1.09 \\
47 & 0.125 & 0.733 & 0.17 \\
48 & 0.271 & 0.49 & 0.55 \\
49 & 0.252 & 0.514 & 0.49 \\
50 & 0.4 & 0.668 & 0.60 \\
51 & 0.229 & 0.642 & 0.36 \\
52 & 0.302 & 0.498 & 0.61 \\
53 & 0.263 & 0.99 & 0.20 \\
54 & 0.658 & 0.411 & 1.61 \\
55 & 0.25 & 0.289 & 0.87 \\
56 & 0.449 & 0.557 & 0.81 \\
57 & 0.538 & 0.666 & 0.81 \\
58 & 0.397 & 0.517 & 0.77 \\
59 & 0.284 & 0.413 & 0.69 \\
60 & 0.284 & 0.664 & 0.43 \\
61 & 0.302 & 0.691 & 0.44 \\
62 & 0.442 & 0.797 & 0.55 \\
63 & 0.415 & 1.22 & 0.34 \\
64 & 0.342 & 0.772 & 0.44 \\
65 & 0.19 & 0.19 & 1.00 \\
66 & 0.697 & 0.637 & 1.09 \\
67 & 0.849 & 0.533 & 1.59 \\
68 & 0.561 & 0.2 & 2.81 \\
69 & 0.429 & 0.386 & 1.11 \\
70 & 0.503 & 0.201 & 2.50 \\
71 & 0.774 & 0.281 & 2.75 \\
72 & 1.099 & 0.486 & 2.26 \\
73 & 0.324 & 0.344 & 0.94 \\
74 & 0.322 & 0.256 & 1.26 \\
75 & 1.211 & 0.527 & 2.30 \\
76 & 0.615 & 0.365 & 1.68 \\
77 & 0.562 & 0.29 & 1.94 \\
78 & 0.627 & 0.304 & 2.06 \\
79 & 0.488 & 0.298 & 1.64 \\
80 & 0.463 & 0.286 & 1.62 \\
81 & 0.333 & 0.219 & 1.52 \\
82 & 0.365 & 0.326 & 1.12 \\
83 & 0.573 & 0.344 & 1.67 \\
84 & 0.502 & 0.275 & 1.83 \\
85 & 0.95 & 0.35 & 2.71 \\
86 & 0.518 & 0.419 & 1.24 \\
87 & 0.597 & 0.465 & 1.28 \\
88 & 0.594 & 0.28 & 2.12 \\
89 & 0.718 & 0.302 & 2.38 \\
90 & 0.723 & 0.366 & 1.98 \\
91 & 0.553 & 0.365 & 1.52 \\
92 & 0.427 & 0.271 & 1.58 \\
93 & 0.469 & 0.25 & \\
\hline 3 & & & \\
53 & 0.37 &
\end{tabular}




\begin{tabular}{|c|c|c|c|}
\hline 94 & 0.427 & 0.317 & 1.35 \\
\hline 95 & 0.695 & 0.347 & 2.00 \\
\hline 96 & 0.569 & 0.42 & 1.35 \\
\hline 97 & 0.619 & 0.423 & 1.46 \\
\hline 98 & 0.367 & 0.198 & 1.85 \\
\hline 99 & 0.523 & 0.261 & 2.00 \\
\hline 100 & 0.556 & 0.167 & 3.33 \\
\hline 101 & 0.55 & 0.296 & 1.86 \\
\hline 102 & 0.568 & 0.392 & 1.45 \\
\hline 103 & 0.799 & 0.246 & 3.25 \\
\hline 104 & 0.393 & 0.225 & 1.75 \\
\hline 105 & 0.642 & 0.332 & 1.93 \\
\hline 106 & 0.597 & 0.205 & 2.91 \\
\hline 107 & 0.314 & 0.177 & 1.77 \\
\hline 108 & 0.558 & 0.243 & 2.30 \\
\hline 109 & 0.405 & 0.28 & 1.45 \\
\hline 110 & 0.61 & 0.498 & 1.22 \\
\hline 111 & 0.701 & 0.661 & 1.06 \\
\hline 112 & 0.475 & 0.491 & 0.97 \\
\hline 113 & 0.471 & 0.244 & 1.93 \\
\hline 114 & 0.234 & 0.215 & 1.09 \\
\hline 115 & 0.48 & 0.24 & 2.00 \\
\hline 116 & 0.574 & 0.317 & 1.81 \\
\hline 117 & 0.61 & 0.273 & 2.23 \\
\hline 118 & 0.557 & 0.173 & 3.22 \\
\hline 119 & 0.664 & 0.419 & 1.58 \\
\hline 120 & 0.402 & 0.311 & 1.29 \\
\hline 121 & 0.656 & 0.35 & 1.87 \\
\hline 122 & 0.488 & 0.332 & 1.47 \\
\hline 123 & 0.658 & 0.393 & 1.67 \\
\hline 124 & 0.762 & 0.281 & 2.71 \\
\hline 125 & 0.992 & 0.61 & 1.63 \\
\hline 126 & 0.419 & 0.257 & 1.63 \\
\hline 127 & 0.619 & 0.209 & 2.96 \\
\hline 128 & 0.384 & 0.312 & 1.23 \\
\hline 129 & 0.303 & 0.238 & 1.27 \\
\hline 130 & 0.389 & 0.281 & 1.38 \\
\hline 131 & 0.583 & 0.369 & 1.58 \\
\hline 132 & 0.671 & 0.283 & 2.37 \\
\hline 133 & 0.345 & 0.155 & 2.23 \\
\hline 134 & 0.349 & 0.277 & 1.26 \\
\hline 135 & 0.658 & 0.363 & 1.81 \\
\hline 136 & 0.694 & 0.22 & 3.15 \\
\hline 137 & 0.38 & 0.215 & 1.77 \\
\hline 138 & 0.324 & 0.177 & 1.83 \\
\hline 139 & 0.847 & 0.55 & 1.54 \\
\hline 140 & 0.636 & 0.48 & 1.33 \\
\hline 141 & 0.548 & 0.221 & 2.48 \\
\hline 142 & 0.715 & 0.437 & 1.64 \\
\hline 143 & 0.606 & 0.427 & 1.42 \\
\hline
\end{tabular}




$\begin{array}{cccc}144 & 0.781 & 0.252 & 3.10 \\ 145 & 0.691 & 0.24 & 2.88 \\ 147 & 0.738 & 0.264 & 2.79 \\ 148 & 0.473 & 0.155 & 3.05 \\ 149 & 0.72 & 0.346 & 2.08 \\ 150 & 0.563 & 0.392 & 1.44 \\ 151 & 0.454 & 0.332 & 1.37 \\ \text { Median } & 0.643 & 0.3615 & 1.75 \\ \text { STD } & 0.267286 & 0.041719 & 0.54\end{array}$

2. Lower friable shale, Russell core, $\mathrm{N}=150$

\begin{tabular}{|c|c|c|c|}
\hline Frequency & Length & Width & $\begin{array}{l}\mathrm{L} / \mathrm{W} \\
\text { Ratio }\end{array}$ \\
\hline 1 & 0.778 & 0.423 & 1.84 \\
\hline 2 & 0.748 & 0.424 & 1.76 \\
\hline 3 & 0.792 & 0.491 & 1.61 \\
\hline 4 & 0.894 & 0.373 & 2.40 \\
\hline 5 & 0.615 & 0.451 & 1.36 \\
\hline 6 & 0.991 & 0.323 & 3.07 \\
\hline 7 & 0.446 & 0.275 & 1.62 \\
\hline 8 & 0.762 & 0.312 & 2.44 \\
\hline 9 & 0.505 & 0.271 & 1.86 \\
\hline 10 & 0.534 & 0.428 & 1.25 \\
\hline 11 & 0.567 & 0.364 & 1.56 \\
\hline 12 & 0.429 & 0.427 & 1.00 \\
\hline 13 & 1.042 & 0.501 & 2.08 \\
\hline 14 & 1.2 & 0.796 & 1.51 \\
\hline 15 & 0.969 & 0.55 & 1.76 \\
\hline 16 & 0.85 & 0.518 & 1.64 \\
\hline 17 & 0.979 & 0.543 & 1.80 \\
\hline 18 & 0.89 & 0.511 & 1.74 \\
\hline 19 & 0.445 & 0.357 & 1.25 \\
\hline 20 & 0.552 & 0.209 & 2.64 \\
\hline 21 & 0.827 & 0.416 & 1.99 \\
\hline 22 & 0.662 & 0.415 & 1.60 \\
\hline 23 & 0.787 & 0.49 & 1.61 \\
\hline 24 & 0.643 & 0.435 & 1.48 \\
\hline 25 & 0.899 & 0.532 & 1.69 \\
\hline 26 & 0.764 & 0.532 & 1.43 \\
\hline 27 & 0.928 & 1.115 & 0.83 \\
\hline 28 & 0.76 & 0.485 & 1.57 \\
\hline 29 & 0.776 & 0.493 & 1.57 \\
\hline 30 & 1.012 & 1.049 & 0.96 \\
\hline 31 & 0.648 & 0.526 & 1.23 \\
\hline 32 & 0.455 & 0.499 & 0.91 \\
\hline 33 & 0.917 & 0.502 & 1.83 \\
\hline 34 & 0.968 & 0.571 & 1.70 \\
\hline 35 & 0.671 & 0.532 & 1.26 \\
\hline 36 & 0.986 & 0.657 & 1.50 \\
\hline 37 & 1.07 & 0.492 & 2.17 \\
\hline
\end{tabular}




\begin{tabular}{rrrr}
38 & 0.984 & 0.535 & 1.84 \\
39 & 0.836 & 0.369 & 2.27 \\
40 & 0.527 & 0.387 & 1.36 \\
41 & 0.699 & 0.523 & 1.34 \\
42 & 0.508 & 0.805 & 0.63 \\
43 & 0.595 & 1.11 & 0.54 \\
44 & 0.706 & 0.611 & 1.16 \\
45 & 0.324 & 0.712 & 0.46 \\
46 & 0.323 & 0.349 & 0.93 \\
47 & 0.415 & 0.847 & 0.49 \\
48 & 0.693 & 0.632 & 1.10 \\
49 & 0.39 & 0.927 & 0.42 \\
50 & 0.488 & 0.824 & 0.59 \\
51 & 0.339 & 0.454 & 0.75 \\
52 & 0.605 & 0.621 & 0.97 \\
53 & 0.407 & 0.778 & 0.52 \\
54 & 0.385 & 0.722 & 0.53 \\
55 & 0.463 & 0.402 & 1.15 \\
56 & 0.766 & 0.502 & 1.53 \\
57 & 0.438 & 0.289 & 1.52 \\
58 & 0.616 & 0.288 & 2.14 \\
59 & 0.524 & 0.354 & 1.48 \\
60 & 0.272 & 0.28 & 0.97 \\
61 & 0.341 & 0.236 & 1.44 \\
62 & 0.516 & 0.302 & 1.71 \\
63 & 0.25 & 0.295 & 0.84 \\
64 & 0.9 & 0.511 & 1.76 \\
65 & 1.463 & 0.459 & 3.19 \\
66 & 0.531 & 0.373 & 1.42 \\
67 & 0.884 & 0.482 & 1.83 \\
68 & 0.672 & 0.451 & 1.49 \\
69 & 0.452 & 0.335 & 1.35 \\
70 & 1.034 & 0.825 & 1.25 \\
71 & 0.498 & 0.2 & 2.49 \\
72 & 0.793 & 0.376 & 2.11 \\
73 & 0.509 & 0.392 & 1.30 \\
74 & 0.629 & 0.346 & 1.82 \\
75 & 0.457 & 0.277 & 1.65 \\
76 & 0.432 & 0.281 & 1.54 \\
77 & 0.558 & 0.384 & 1.45 \\
78 & 0.585 & 0.317 & 1.85 \\
79 & 0.379 & 0.275 & 1.39 \\
80 & 0.978 & 0.752 & 1.30 \\
81 & 0.589 & 0.185 & 3.18 \\
82 & 0.656 & 0.377 & 1.74 \\
83 & 0.337 & 0.274 & 1.23 \\
84 & 0.38 & 0.315 & 1.21 \\
85 & 0.319 & 0.243 & 1.31 \\
86 & 0.485 & 0.224 & 2.17 \\
87 & 0.405 & 0.317 & 1.28 \\
\hline & & &
\end{tabular}




$\begin{array}{rrrr}88 & 0.607 & 0.208 & 2.92 \\ 89 & 0.585 & 0.324 & 1.81 \\ 90 & 0.451 & 0.376 & 1.20 \\ 91 & 0.494 & 0.335 & 1.47 \\ 92 & 0.555 & 0.431 & 1.29 \\ 93 & 0.498 & 0.323 & 1.54 \\ 94 & 0.675 & 0.275 & 2.45 \\ 95 & 0.756 & 0.433 & 1.74 \\ 96 & 0.787 & 0.379 & 2.08 \\ 97 & 0.857 & 0.317 & 2.70 \\ 98 & 0.541 & 0.312 & 1.73 \\ 99 & 0.442 & 0.356 & 1.24 \\ 100 & 0.42 & 0.21 & 2.00 \\ 101 & 0.292 & 0.199 & 1.48 \\ 102 & 0.638 & 0.24 & 2.66 \\ 103 & 0.335 & 0.271 & 1.24 \\ 104 & 0.245 & 0.182 & 1.35 \\ 105 & 0.326 & 0.142 & 2.30 \\ 106 & 0.386 & 0.346 & 1.12 \\ 107 & 0.629 & 0.347 & 1.81 \\ 108 & 1.148 & 0.528 & 2.17 \\ 109 & 0.435 & 0.359 & 1.21 \\ 110 & 0.557 & 0.551 & 1.01 \\ 111 & 0.303 & 0.317 & 0.96 \\ 112 & 0.277 & 0.345 & 0.80 \\ 113 & 0.17 & 0.473 & 0.36 \\ 114 & 0.448 & 0.541 & 0.83 \\ 115 & 0.335 & 0.346 & 0.97 \\ 116 & 0.28 & 0.199 & 1.41 \\ 117 & 0.111 & 0.247 & 0.45 \\ 118 & 0.159 & 0.626 & 0.25 \\ 119 & 0.355 & 0.594 & 0.60 \\ 120 & 0.324 & 0.354 & 0.91 \\ 121 & 0.221 & 0.302 & 0.73 \\ 122 & 0.229 & 0.343 & 0.67 \\ 123 & 0.252 & 0.501 & 0.50 \\ 124 & 0.281 & 0.617 & 0.46 \\ 125 & 0.313 & 0.345 & 0.91 \\ 126 & 0.302 & 0.435 & 0.69 \\ 127 & 0.238 & 0.402 & 0.59 \\ 128 & 0.3 & 0.616 & 0.49 \\ 129 & 0.359 & 0.436 & 0.82 \\ 130 & 0.257 & 0.368 & 0.70 \\ 131 & 0.281 & 0.596 & 0.47 \\ 132 & 0.383 & 0.872 & 0.44 \\ 133 & 0.382 & 0.601 & 0.63 \\ 134 & 0.245 & 0.41 & 0.60 \\ 135 & 0.28 & 0.533 & 0.53 \\ 136 & 0.348 & 0.622 & 0.56 \\ 137 & 0.333 & 1.055 & 0.32\end{array}$




$\begin{array}{rrrr}138 & 0.445 & 0.567 & 0.78 \\ 139 & 0.24 & 0.261 & 0.92 \\ 140 & 0.125 & 0.612 & 0.20 \\ 141 & 0.332 & 0.503 & 0.66 \\ 142 & 1.307 & 0.617 & 2.12 \\ 143 & 1.255 & 0.479 & 2.62 \\ 144 & 1.092 & 0.435 & 2.51 \\ 145 & 0.979 & 0.665 & 1.47 \\ 146 & 1.199 & 0.519 & 2.31 \\ 147 & 1.271 & 0.576 & 2.21 \\ 148 & 0.906 & 0.596 & 1.52 \\ 149 & 0.907 & 0.833 & 1.09 \\ 150 & 1.115 & 0.742 & 1.50 \\ \text { Average } & 0.9465 & 0.5825 & 1.67 \\ \text { STD } & 0.238295 & 0.225567 & 0.24\end{array}$

3. Upper friable shale, Billings Bridge core, $n=150$

$\begin{array}{cccc}\text { Frequency } & \text { Length } & \text { Width } & \text { Ratio } \\ 1 & 0.217 & 0.174 & 1.25 \\ 2 & 0.224 & 0.16 & 1.40 \\ 3 & 0.19 & 0.153 & 1.24 \\ 4 & 0.318 & 0.139 & 2.29 \\ 5 & 0.243 & 0.141 & 1.72 \\ 6 & 0.185 & 0.101 & 1.83 \\ 7 & 0.209 & 0.086 & 2.43 \\ 8 & 0.129 & 0.084 & 1.54 \\ 9 & 0.2 & 0.108 & 1.85 \\ 10 & 0.192 & 0.093 & 2.06 \\ 11 & 0.118 & 0.059 & 2 \\ 12 & 0.09 & 0.071 & 1.27 \\ 13 & 0.197 & 0.103 & 1.91 \\ 14 & 0.229 & 0.097 & 2.36 \\ 15 & 0.154 & 0.099 & 1.56 \\ 16 & 0.103 & 0.066 & 1.56 \\ 17 & 0.084 & 0.079 & 1.06 \\ 18 & 0.118 & 0.042 & 2.81 \\ 19 & 0.094 & 0.084 & 1.12 \\ 20 & 0.149 & 0.069 & 2.16 \\ 21 & 0.189 & 0.139 & 1.36 \\ 22 & 0.31 & 0.053 & 5.85 \\ 23 & 0.234 & 0.098 & 2.39 \\ 24 & 0.09 & 0.049 & 1.84 \\ 25 & 0.174 & 0.056 & 3.11 \\ 26 & 0.435 & 0.112 & 3.88 \\ 27 & 0.202 & 0.056 & 3.61 \\ 28 & 0.243 & 0.049 & 4.96 \\ 29 & 0.161 & 0.051 & 3.16 \\ 30 & 0.219 & 0.099 & 2.21\end{array}$




$\begin{array}{lccc}31 & 0.118 & 0.075 & 1.57 \\ 32 & 0.22 & 0.099 & 2.23 \\ 33 & 0.22 & 0.042 & 5.24 \\ 34 & 0.035 & 0.056 & 0.63 \\ 35 & 0.399 & 0.133 & 3.00 \\ 36 & 0.152 & 0.085 & 1.79 \\ 37 & 0.301 & 0.138 & 2.18 \\ 38 & 0.348 & 0.104 & 3.35 \\ 39 & 0.152 & 0.089 & 1.71 \\ 40 & 0.167 & 0.054 & 3.09 \\ 41 & 0.044 & 0.037 & 1.19 \\ 42 & 0.188 & 0.118 & 1.59 \\ 43 & 0.139 & 0.063 & 2.21 \\ 44 & 0.283 & 0.119 & 2.39 \\ 45 & 0.125 & 0.073 & 1.71 \\ 46 & 0.133 & 0.044 & 3.02 \\ 47 & 0.143 & 0.073 & 1.96 \\ 48 & 0.183 & 0.108 & 1.69 \\ 49 & 0.093 & 0.057 & 1.63 \\ 50 & 0.149 & 0.098 & 1.52 \\ 51 & 0.093 & 0.079 & 1.18 \\ 52 & 0.152 & 0.035 & 4.34 \\ 53 & 0.168 & 0.039 & 4.31 \\ 54 & 0.139 & 0.088 & 1.58 \\ 55 & 0.2 & 0.123 & 1.63 \\ 56 & 0.214 & 0.158 & 1.35 \\ 57 & 0.26 & 0.157 & 1.66 \\ 58 & 0.204 & 0.07 & 2.91 \\ 59 & 0.125 & 0.056 & 2.23 \\ 60 & 0.14 & 0.07 & 2.00 \\ 61 & 0.23 & 0.094 & 2.45 \\ 62 & 0.112 & 0.081 & 1.38 \\ 63 & 0.176 & 0.077 & 2.29 \\ 64 & 0.225 & 0.198 & 1.14 \\ 65 & 0.119 & 0.115 & 1.03 \\ 66 & 0.203 & 0.04 & 5.08 \\ 67 & 0.19 & 0.03 & 6.34 \\ 68 & 0.154 & 0.07 & 2.20 \\ 69 & 0.125 & 0.037 & 3.38 \\ 70 & 0.157 & 0.029 & 5.41 \\ 71 & 0.245 & 0.064 & 3.83 \\ 72 & 0.195 & 0.232 & 0.84 \\ 73 & 0.146 & 0.084 & 1.74 \\ 74 & 0.143 & 0.085 & 1.68 \\ 70 & 0.157 & 0.101 & 1.55 \\ 79 & 0.199 & 0.089 & 2.24 \\ 79 & 0.181 & 0.053 & 3.42 \\ 75 & 0.232 & 0.051 & 4.55 \\ 73 & 0.125 & 0.06 & 2.08 \\ 73 & 0.162 & 1.23\end{array}$




$\begin{array}{lccc}81 & 0.188 & 0.104 & 1.81 \\ 82 & 0.218 & 0.039 & 5.59 \\ 83 & 0.189 & 0.035 & 5.40 \\ 84 & 0.217 & 0.177 & 1.23 \\ 85 & 0.347 & 0.215 & 1.61 \\ 86 & 0.215 & 0.112 & 1.92 \\ 87 & 0.054 & 0.14 & 0.39 \\ 88 & 0.053 & 0.174 & 0.30 \\ 89 & 0.098 & 0.087 & 1.13 \\ 90 & 0.069 & 0.067 & 1.03 \\ 91 & 0.059 & 0.084 & 0.70 \\ 92 & 0.029 & 0.174 & 0.17 \\ 93 & 0.051 & 0.165 & 0.31 \\ 94 & 0.044 & 0.135 & 0.33 \\ 95 & 0.084 & 0.202 & 0.42 \\ 96 & 0.084 & 0.074 & 1.14 \\ 97 & 0.044 & 0.281 & 0.15 \\ 98 & 0.114 & 0.196 & 0.58 \\ 99 & 0.091 & 0.197 & 0.46 \\ 100 & 0.056 & 0.191 & 0.29 \\ 101 & 0.093 & 0.189 & 0.49 \\ 102 & 0.108 & 0.196 & 0.55 \\ 103 & 0.15 & 0.104 & 1.44 \\ 104 & 0.059 & 0.34 & 0.17 \\ 105 & 0.167 & 0.111 & 1.50 \\ 106 & 0.181 & 0.135 & 1.34 \\ 107 & 0.12 & 0.222 & 0.54 \\ 108 & 0.128 & 0.158 & 0.81 \\ 109 & 0.084 & 0.242 & 0.35 \\ 110 & 0.201 & 0.222 & 0.91 \\ 111 & 0.081 & 0.084 & 0.96 \\ 112 & 0.066 & 0.054 & 1.23 \\ 113 & 0.274 & 0.084 & 3.26 \\ 114 & 0.135 & 0.091 & 1.48 \\ 115 & 0.143 & 0.06 & 2.38 \\ 116 & 0.116 & 0.084 & 1.38 \\ 117 & 0.125 & 0.16 & 0.78 \\ 118 & 0.091 & 0.066 & 1.39 \\ 119 & 0.143 & 0.115 & 1.24 \\ 120 & 0.306 & 0.097 & 3.15 \\ 121 & 0.221 & 0.139 & 1.59 \\ 122 & 0.181 & 0.084 & 2.15 \\ 123 & 0.176 & 0.113 & 1.56 \\ 124 & 0.22 & 0.064 & 3.44 \\ 125 & 0.215 & 0.042 & 5.12 \\ 126 & 0.242 & 0.059 & 4.12 \\ 127 & 0.084 & 0.029 & 2.90 \\ 128 & 0.367 & 0.16 & 2.29 \\ 129 & 0.295 & 0.064 & 4.61 \\ 130 & 0.139 & 0.042 & 3.31\end{array}$




$\begin{array}{cccc}131 & 0.241 & 0.042 & 5.74 \\ 132 & 0.255 & 0.071 & 3.59 \\ 133 & 0.149 & 0.029 & 5.14 \\ 134 & 0.36 & 0.05 & 7.2 \\ 135 & 0.137 & 0.089 & 1.54 \\ 136 & 0.174 & 0.09 & 1.93 \\ 137 & 0.223 & 0.169 & 1.32 \\ 138 & 0.241 & 0.114 & 2.11 \\ 139 & 0.21 & 0.106 & 1.98 \\ 140 & 0.106 & 0.039 & 2.72 \\ 141 & 0.265 & 0.044 & 6.02 \\ 142 & 0.341 & 0.083 & 4.11 \\ 143 & 0.229 & 0.042 & 5.45 \\ 144 & 0.345 & 0.104 & 3.32 \\ 145 & 0.09 & 0.069 & 1.30 \\ 146 & 0.07 & 0.042 & 1.67 \\ 147 & 0.33 & 0.103 & 3.20 \\ 148 & 0.14 & 0.062 & 2.26 \\ 149 & 0.104 & 0.044 & 2.36 \\ 150 & 0.262 & 0.118 & 2.22 \\ 151 & 0.382 & 0.112 & 3.41 \\ \text { Average } & 0.174636 & 0.100285 & 2.24 \\ \text { STD } & 0.081903 & 0.05514 & 1.46 \\ \text { Skewness } & 0.672582 & 1.312153 & 1.12\end{array}$




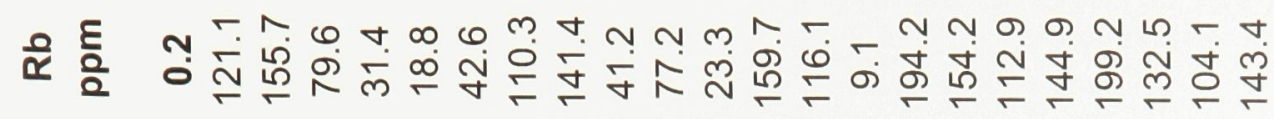

은 흥

z

를 응

듬 ๆ

- E n m m

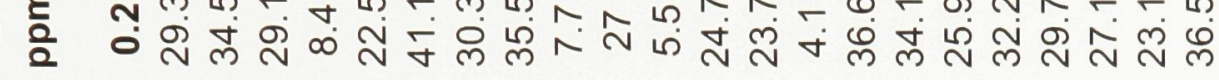

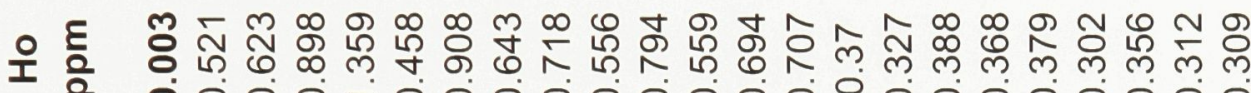

壱

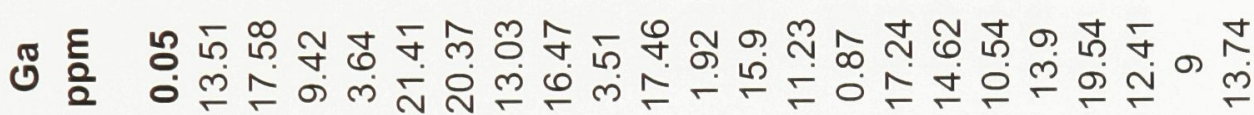

उ

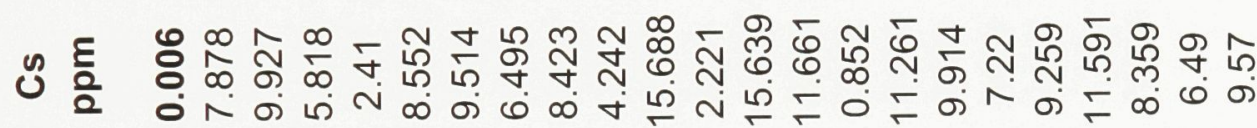

ப

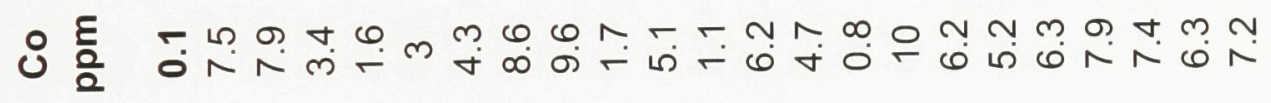

Ј

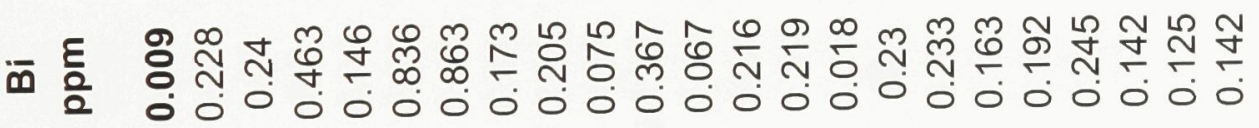

๓

๓

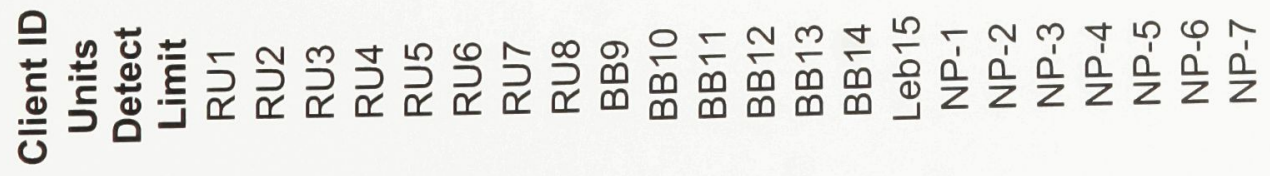




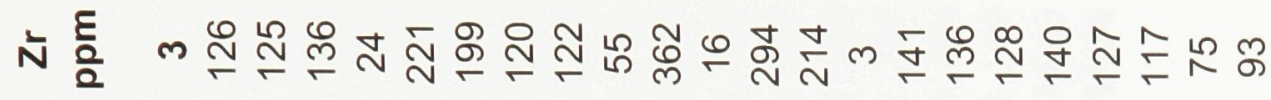

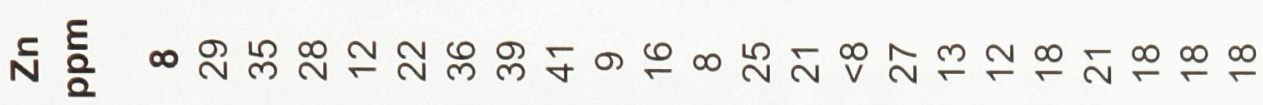

$>$ ₹

उ

$>$ 듬 으 움

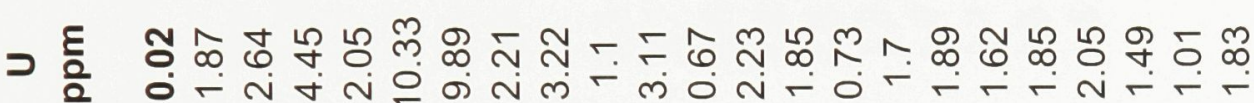

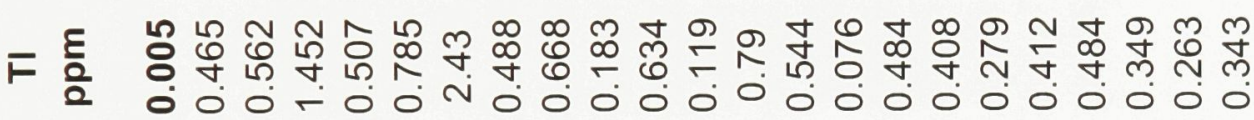

i=

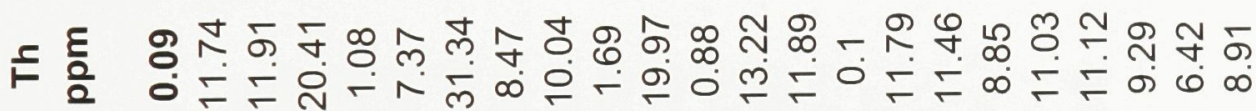



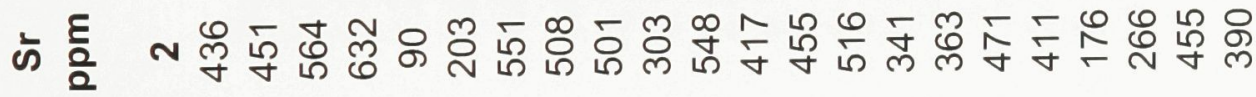

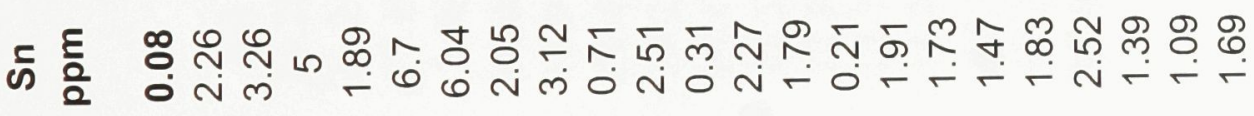

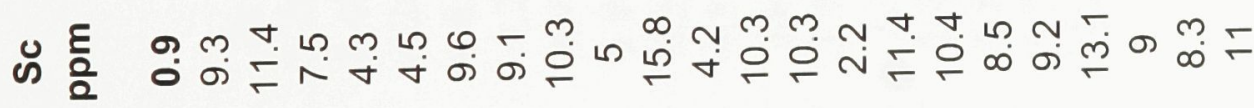

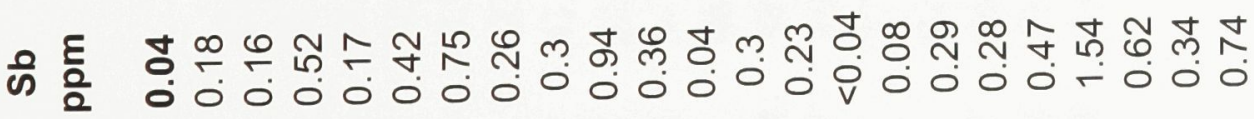

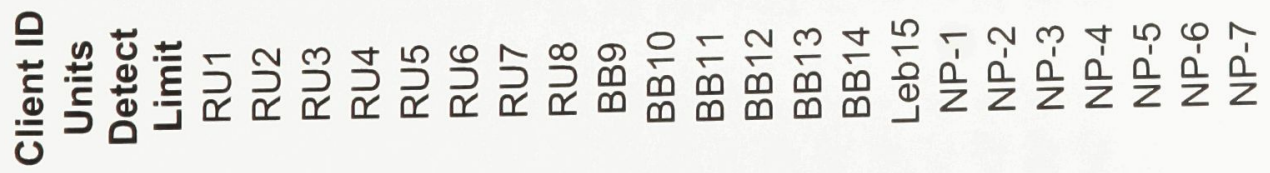




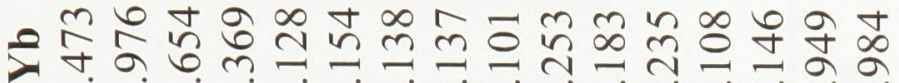
-

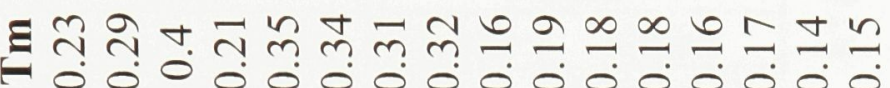

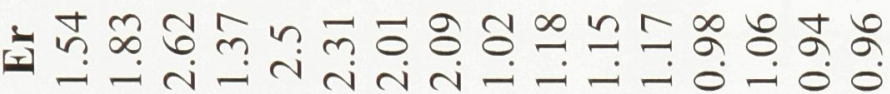

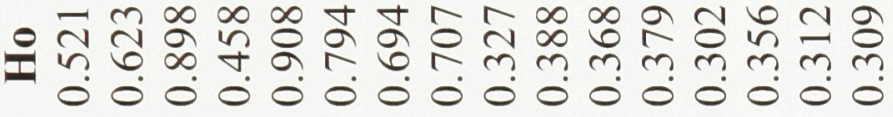

穴

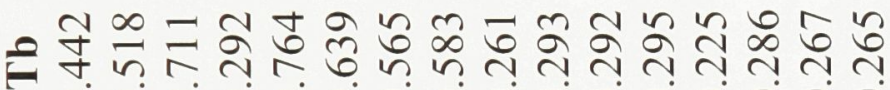
0000000000000000

ت

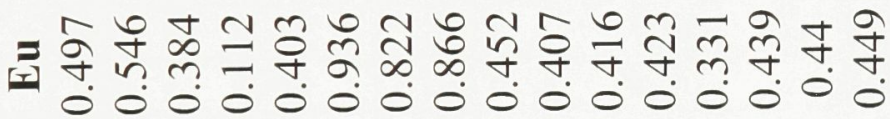
ש

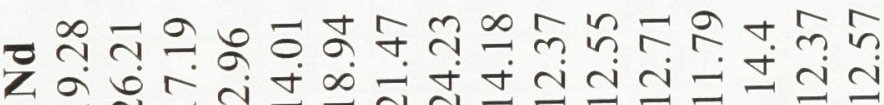
ن ช 再孚

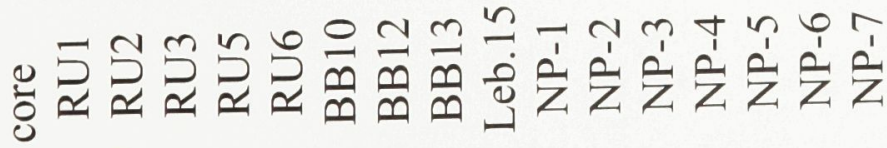


APPENDIX D: HFSE, LILE and REE dataset of friable and compacted shales, Verulam formation.

\begin{tabular}{|c|c|c|c|c|c|c|c|c|}
\hline Lithology & & riable I & lica-Sh & & & $\begin{array}{r}\text { Comp } \\
\text { sh }\end{array}$ & ted - & \\
\hline Locality & & $\begin{array}{l}\text { sell } \\
\text { re }\end{array}$ & Billin & $\begin{array}{l}\text { s Bridge } \\
\text { ore }\end{array}$ & שั" & $\begin{array}{l}\text { Leberton } \\
\text { Core }\end{array}$ & $\begin{array}{c}\text { Nepean } \\
\text { Pt. }\end{array}$ & 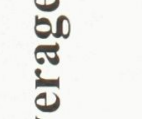 \\
\hline Zone & Lower & Upper & Lower & Upper & $\sum$ & & Section & \\
\hline Sample & $\begin{array}{l}\text { RU3 } \\
\mathrm{N}=1\end{array}$ & $\begin{array}{c}\text { RU5\&6 } \\
\mathrm{N}=2\end{array}$ & $\begin{array}{l}\text { BB10 } \\
\mathrm{N}=1\end{array}$ & $\begin{array}{c}\mathrm{BB} 12 \& 13 \\
\mathrm{~N}=2\end{array}$ & & $\begin{array}{c}\text { Leb. } 15 \\
\mathrm{~N}=1\end{array}$ & $\begin{array}{l}\mathrm{NP}-7 \\
\mathrm{~N}=7\end{array}$ & \\
\hline HFSE & 190.88 & $\begin{array}{l}262.98 \\
(\mathbf{1 0 . 6 2 )}\end{array}$ & 446.31 & $\begin{array}{l}329.65 \\
(\mathbf{6 3 . 1 8}) \\
\end{array}$ & $\begin{array}{r}307.46 \\
(108.54)\end{array}$ & 233.74 & $\begin{array}{l}193.69 \\
(\mathbf{3 3 . 8 2})\end{array}$ & $\begin{array}{l}213.70 \\
(28.29)\end{array}$ \\
\hline LILE & 763 & $\begin{array}{r}169.53 \\
(\mathbf{1 3 8 . 9 7 )}\end{array}$ & 476.58 & $\begin{array}{l}683.15 \\
(27.16)\end{array}$ & $\begin{array}{l}523.07 \\
(264.79)\end{array}$ & 854.29 & $\begin{array}{l}854.90 \\
(\mathbf{1 8 7 . 3 3 )}\end{array}$ & $\begin{array}{c}854.60 \\
(0.43)\end{array}$ \\
\hline$\sum \mathrm{REE}$ & 88.52 & $\begin{array}{l}43.50 \\
(36.92)\end{array}$ & 104.08 & $\begin{array}{c}127.22 \\
(\mathbf{4 . 2 5 )}\end{array}$ & $\begin{array}{l}\mathbf{9 0 . 8 3} \\
(35.33)\end{array}$ & 100.58 & $\begin{array}{l}85.68 \\
(\mathbf{8 . 0 6 )}\end{array}$ & $\begin{array}{r}93.13 \\
(10.53)\end{array}$ \\
\hline $\begin{array}{l}\text { LREE/ } \\
\text { HREE }\end{array}$ & 4.28 & 2.31 & 5.77 & 7.89 & 5.06 & 13.92 & 11.41 & 12.67 \\
\hline $\mathbf{E u} / \mathbf{E} u^{*}$ & 0.09 & 0.10 & 0.23 & 0.20 & 0.16 & 0.23 & 0.22 & 0.23 \\
\hline $\mathrm{Th} / \mathrm{Sc}$ & 2.72 & 2.75 & 1.26 & 1.99 & 1.91 & 1.03 & 0.86 & 0.95 \\
\hline $\mathrm{La} / \mathrm{Th}$ & 0.68 & 0.24 & 0.93 & 2.07 & 0.98 & 2.25 & 2.31 & 2.28 \\
\hline $\mathrm{Cr} / \mathrm{Th}$ & 1.18 & 2.02 & 1.21 & 2.41 & 1.71 & 7.38 & 6.85 & 7.12 \\
\hline $\mathrm{Th} / \mathrm{U}$ & 4.59 & 1.91 & 6.42 & 6.15 & 4.77 & 6.93 & 4.87 & 5.90 \\
\hline $\mathrm{Cr} / \mathrm{Zr}$ & 0.18 & 0.11 & 0.07 & 0.12 & 0.12 & 0.62 & 0.78 & 0.70 \\
\hline $\mathrm{Cr} / \mathrm{V}$ & 1.85 & 1.37 & 0.69 & 0.90 & 1.23 & 1.38 & 1.33 & 1.36 \\
\hline $\mathrm{Cr} / \mathrm{Ni}$ & 3.43 & 4.12 & 2.67 & 2.88 & 3.28 & 3.48 & 3.65 & 3.57 \\
\hline $\mathrm{Cr} / \mathrm{Rb}$ & 2.30 & 0.92 & 0.31 & 0.22 & 0.44 & 0.44 & 0.46 & 0.45 \\
\hline $\mathrm{Zr} / \mathrm{Rb}$ & 1.71 & 8.22 & 4.69 & 1.84 & 4.12 & 0.73 & 0.84 & 0.78 \\
\hline $\mathrm{Ba} / \mathrm{Rb}$ & 1.19 & 0.81 & 0.95 & 0.69 & 0.91 & 1.57 & 2.33 & 1.95 \\
\hline $\mathrm{Ni} / \mathrm{Co}$ & 2.06 & 1.64 & 1.76 & 1.92 & 1.85 & 2.50 & 2.80 & 2.65 \\
\hline $\mathrm{Cu} / \mathrm{Zn}$ & 0.14 & 0.17 & 0.50 & 0.27 & 0.27 & 0.26 & 0.56 & 0.41 \\
\hline $\mathrm{Zr} / \mathrm{Sc}$ & 18.13 & 29.79 & 22.91 & 24.66 & 23.87 & 12.7 & 8.45 & 10.58 \\
\hline $\mathrm{Zr} / \mathrm{Nb}$ & 28.45 & 24.57 & 18.46 & 18.17 & 22.41 & 12.34 & 12.70 & 12.52 \\
\hline $\mathrm{Ba} / \mathrm{Sr}$ & 0.17 & 0.17 & 0.24 & 0.22 & 0.20 & 0.90 & 1.13 & 1.02 \\
\hline $\mathrm{Rb} / \mathrm{Sr}$ & 0.14 & 0.21 & 0.25 & 0.32 & 0.23 & 0.57 & 0.46 & 0.52 \\
\hline $\mathrm{Ba} / \mathrm{Sc}$ & 12.65 & 3.50 & 4.64 & 9.19 & 7.50 & 26.81 & 33.38 & 30.10 \\
\hline $\mathrm{Ba} / \mathrm{Co}$ & 27.91 & 7.00 & 14.37 & 17.30 & 16.65 & 30.56 & 51.77 & 41.17 \\
\hline $\mathrm{La} / \mathrm{Lu}$ & 38.23 & 18.63 & 57.20 & 80.47 & 48.63 & 154.97 & 130.27 & 142.62 \\
\hline $\mathrm{La} / \mathrm{Nd}$ & 0.81 & 0.66 & 0.65 & 1.80 & 0.98 & 0.90 & 1.78 & 1.34 \\
\hline $\mathrm{La} / \mathrm{Sm}$ & 3.23 & 1.28 & 4.21 & 5.76 & 3.62 & 11.57 & 8.35 & 9.96 \\
\hline
\end{tabular}




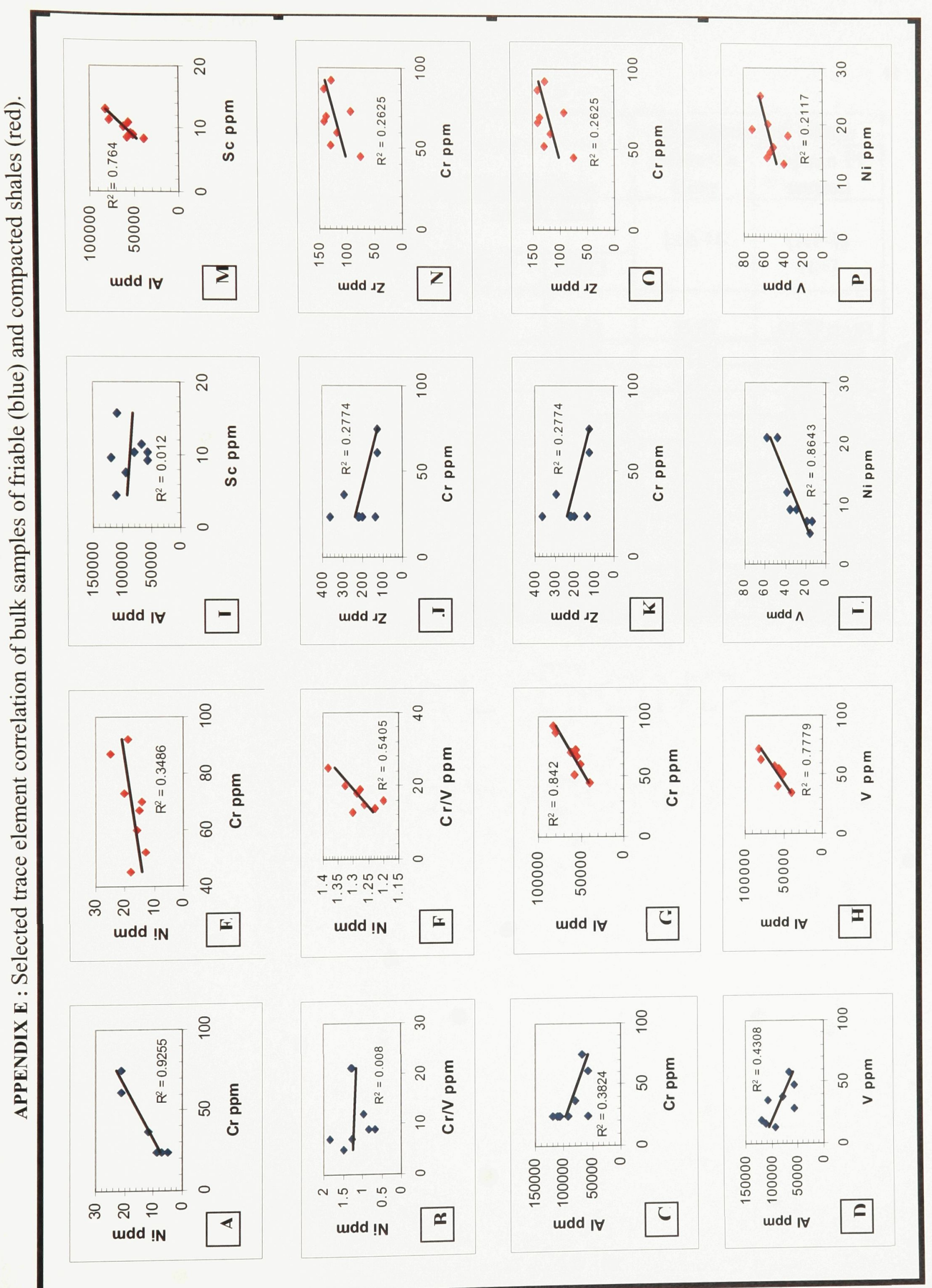


Appendix F. Normalised Major Oxide without LOI.

\begin{tabular}{|c|c|c|c|c|c|c|c|c|}
\hline \multirow{4}{*}{ Oxides } & \multicolumn{6}{|c|}{ Friable mica-shale } & \multicolumn{2}{|c|}{ Compacted shale } \\
\hline & \multicolumn{3}{|c|}{ Russell Core } & \multicolumn{3}{|c|}{ Billings Bridge Core } & \multirow{3}{*}{$\begin{array}{c}\begin{array}{c}\text { Lebreton } \\
\text { Core }\end{array} \\
\text { Leb.16 }\end{array}$} & \multirow{3}{*}{$\begin{array}{c}\begin{array}{c}\text { Nepean Pt. } \\
\text { section }\end{array} \\
\begin{array}{c}\mathrm{NP}-7) \\
\mathrm{N}=7\end{array}\end{array}$} \\
\hline & \multirow{2}{*}{$\begin{array}{c}\text { Upper } \\
\text { Bed }\end{array}$} & \multicolumn{2}{|c|}{ Lower Bed } & \multirow{2}{*}{$\begin{array}{c}\begin{array}{c}\text { Upper } \\
\text { Bed }\end{array} \\
\text { BB10 }\end{array}$} & \multicolumn{2}{|c|}{ Lower Bed } & & \\
\hline & & (RU5) & (RU6) & & $\mathrm{BB} 12$ & BB13 & & \\
\hline $\mathrm{SiO}_{2}$ & 47.04 & 51.78 & 53.72 & 48.02 & 44.86 & 43.52 & 55.82 & $44.58(3.44)$ \\
\hline $\mathrm{Al}_{2} \mathrm{O}_{3}$ & 21.04 & 24.72 & 25.92 & 23.56 & 18.25 & 14.31 & 17.63 & $14.80(0.66)$ \\
\hline $\mathrm{Fe}_{2} \mathrm{O}_{3}$ & 5.29 & 3.86 & 4.33 & 4.38 & 4.31 & 3.42 & 4.33 & $3.06(0.30)$ \\
\hline MnO & 0.02 & 0.02 & 0.01 & 0.02 & 0.03 & 0.04 & 0.04 & $0.04(0.0)$ \\
\hline MgO & 3.83 & 4.02 & 3.85 & 3.44 & 2.90 & 2.52 & 3.51 & $2.20(0.03)$ \\
\hline $\mathrm{CaO}$ & 15.60 & 8.43 & 4.39 & 5.22 & 13.87 & 31.41 & 12.13 & $30.62(2.35)$ \\
\hline $\mathrm{Na}_{2} \mathrm{O}$ & 0.71 & 1.07 & 0.97 & 0.81 & 0.65 & 0.64 & 0.44 & $0.42(0.09)$ \\
\hline $\mathrm{K}_{2} \mathrm{O}$ & 4.96 & 5.87 & 6.32 & 6.14 & 5.26 & 3.46 & 5.21 & $3.36(0.41)$ \\
\hline $\mathrm{TiO}_{2}$ & 0.31 & 0.31 & 0.30 & 0.63 & 0.64 & 0.46 & 0.74 & $0.66(0.0)$ \\
\hline $\mathrm{P}_{2} \mathrm{O}_{5}$ & 0.25 & 0.20 & 0.17 & 0.15 & 0.18 & 0.21 & 0.13 & $0.24(0.06)$ \\
\hline $\mathrm{Cr}_{2} \mathrm{O}_{3}$ & $<0.01$ & 0.01 & $<0.01$ & $<0.01$ & $<0.01$ & $<0.01$ & 0.01 & $<0.01$ \\
\hline TOTAL & 99.06 & 100.00 & 99.99 & 100.00 & 100.00 & 100.00 & 99.99 & 99.99 \\
\hline
\end{tabular}

